FIAN/TD-2/06

ITEP/TH-107/05

\title{
Instantons and Merons in Matrix Models
}

\author{
A.Alexandrov ${ }^{\mathrm{a}}$ \\ ITEP, Moscow, Russia \\ A. Mironov ${ }^{\mathrm{b}}$ \\ Lebedev Physics Institute and ITEP, Moscow, Russia
}

A.Morozov

ITEP, Moscow, Russia

\begin{abstract}
Various branches of matrix model partition function can be represented as intertwined products of universal elementary constituents: Gaussian partition functions $Z_{G}$ and Kontsevich $\tau$-functions $Z_{K}$. In physical terms, this decomposition is the matrix-model version of multi-instanton and multi-meron configurations in Yang-Mills theories. Technically, decomposition formulas are related to representation theory of algebras of Krichever-Novikov type on families of spectral curves with additional Seiberg-Witten structure. Representations of these algebras are encoded in terms of "the global partition functions". They interpolate between $Z_{G}$ and $Z_{K}$ associated with different singularities on spectral Riemann surfaces. This construction is nothing but M-theory-like unification of various matrix models with explicit and representative realization of dualities.
\end{abstract}

\footnotetext{
${ }^{\mathrm{a}}$ E-mail: al@itep.ru

${ }^{\mathrm{b}}$ E-mail: mironov@itep.ru; mironov@lpi.ru

${ }^{\mathrm{c}}$ E-mail: morozov@itep.ru
} 


\section{Contents}

$1 \quad$ Introduction. The origin and the essence of decomposition formulas 4

1.1 Two decomposition formulas . . . . . . . . . . . . . . . . . . . . 4

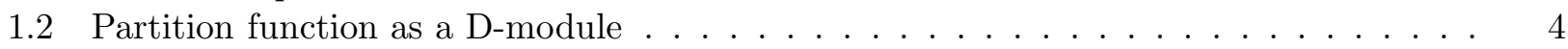

1.3 Krichever-Novikov tvpe algebra and the $x$-product $\ldots \ldots \ldots \ldots \ldots$

1.4 Seiberg-Witten structure and the $*$-product $\ldots \ldots \ldots \ldots \ldots$. . . . . . . . . 6

1.5 Local Virasoro constraints . . . . . . . . . . . . . . . . . . . . . . . . . . 6

1.6 Generic construction. Summary . . . . . . . . . . . . . . . . . . . 7

\begin{tabular}{|lll}
2 & Summary of the Gaussian example & 8
\end{tabular}

2.1 Ingredients of the construction $\ldots \ldots \ldots \ldots \ldots$

2.2 Familiar special functions . . . . . . . . . . . . . . . . . . . . . . . 9

2.3 Web of dualities between matrix models . . . . . . . . . . . . . . . . . 11

2.4 Moment variables . . . . . . . . . . . . . . . . . . . . . . . . . 12

2.4 .1 Changing local parameter . . . . . . . . . . . . . . . . . . . 12

2.4.2 Moment variables for the standard genus expansion of $Z \mathrm{~d}$. . . . . . . . . . . 13

2.5 Partition functions: genus and polvnomial expansions $\ldots \ldots \ldots \ldots$

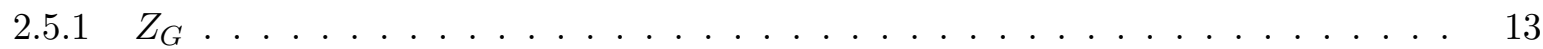

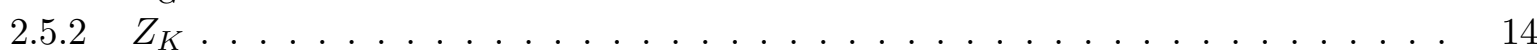

2.5 .3 Non-standard genus expansions . . . . . . . . . . . . . . . . . . . 15

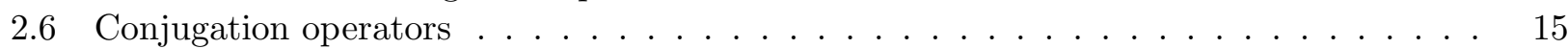

2.7 Summarv: Relations between special functions $\ldots \ldots \ldots \ldots$. . . . . . . . 17

$\begin{array}{lll}3 & Z_{K}(\tau) \rightarrow Z_{K}(\tilde{\tau}) & 19\end{array}$

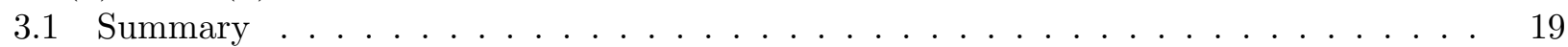

3.1 .1 Comments on the derivation . . . . . . . . . . . . . . . . . . . 20

3.1 .2 Comments on generic change of variables . . . . . . . . . . . . . 22

\begin{tabular}{|lll}
4 & $Z_{G}(t) \rightarrow Z_{C}(\tilde{t})$ & $\mathbf{2 4}$
\end{tabular}

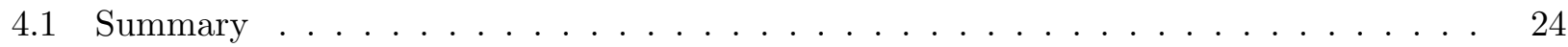

4.2 Comments . . . . . . . . . . . . . . . . . . . . . . . . . 24

5 Proiections of Z(T.S)

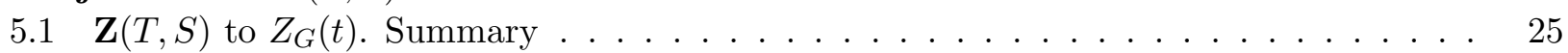

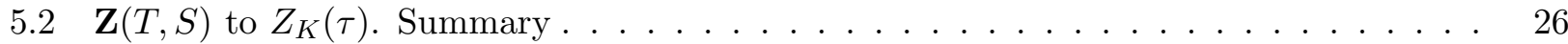

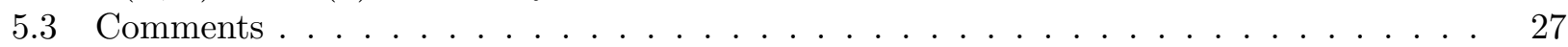

$6 \quad Z_{G}(t) \rightarrow Z_{K}\left(\tau_{+}\right) Z_{K}\left(\tau_{-}\right.$

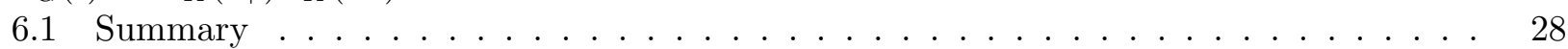

6.2 Comments . . . . . . . . . . . . . . . . . . . . . . . 29

$\begin{array}{lll}7 & Z_{\triangle C K M} \text { and recoverv of Kostov's formula } & \mathbf{3 0}\end{array}$

$7.1 Z_{G}(t) \rightarrow Z_{K}\left(\tau_{\perp}\right) Z_{K}\left(\tau_{-}\right)$in the non-svmmetric case. Summarv $\ldots \ldots \ldots . . . .30$

7.2 Kostov's formula and ACKM polvnomial decomposition $\ldots \ldots \ldots \ldots 31$

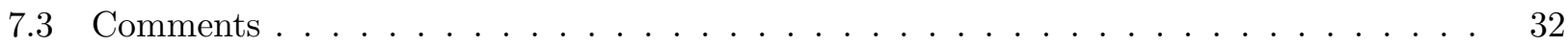

8 Decomposition formula for complex matrix model $\quad 34$

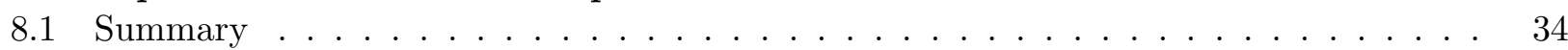

8.2 Comments on moment variables for $Z_{d} \ldots \ldots \ldots \ldots \ldots$ 
\begin{tabular}{|ll|}
\hline Appendix I & Explicit expansions of partition functions \\
\hline
\end{tabular}

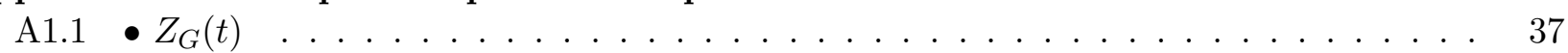

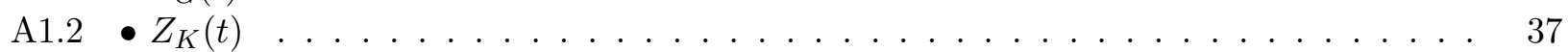

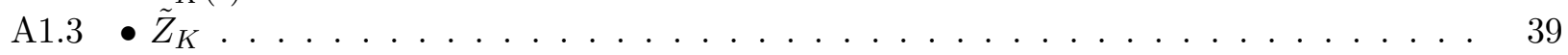

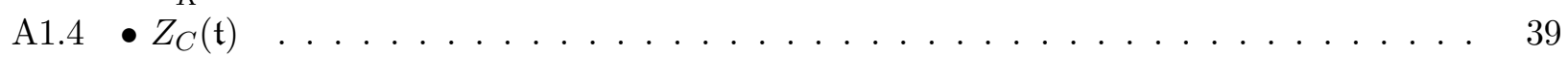

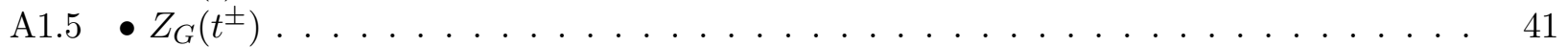

\begin{tabular}{|lll}
\hline Appendix II & Explicit checks of relations between various partition functions & 46
\end{tabular}

A2.1 Experimental check of the decomposition formula . . . . . . . . . . . . . 46

A2.2 Non-trivial dependence on $t_{0}$ and the decomposition formula . . . . . . . . . . . . 47

A2.3 Check of the decomposition formula in terms of ACKM moments . . . . . . . . . . 48

A2.4 Check of the decomposition formula for $Z_{C} \longrightarrow Z_{K} \tilde{Z}_{K} \ldots \ldots \ldots$. . . . . . . . 49 


\section{Introduction. The origin and the essence of decomposition for- mulas}

This paper is a part of a new attack [1, 2] on the bastions of Hermitean matrix model [3]- [5], began in the recent papers [6]- 14], and targeted at exhaustive (reference-book-like) description of properties of partition function $Z(t)$ - the basic special function of string theory. In the present paper, we study relation between $Z(t)$, the partition function of complex matrix model $Z_{C}$ and the Kontsevich $\tau$-function $Z_{K}$. Moreover, we unify all of them with the use of (an a priori different) interpolating global partition function associated with Virasoro constraints of the Krichever-Novikov type. This M-theory like unification of various matrix models (with the global partition function playing the role of M-theory partition function) provides an explicit realization of dualities [15] (with three types of matrix models playing the role of the five superstring theories).

\subsection{Two decomposition formulas}

In the present paper we address the subject of decomposition formulas. Any branch of partition function, which possesses genus expansion and is parameterized by a hyperelliptic spectral curve $\Sigma_{n}$ with $n$ pairs of ramification points of the second order, $a_{1}^{ \pm}, \ldots, a_{n}^{ \pm}$, can be decomposed into a product of $n$ Gaussian partition functions $Z_{G}$ [16, 1] and, further [17]-[19], into that of $2 n$ Kontsevich $\tau$-functions [20-23], associated with particular ramification points $a_{i}$ :

$$
Z_{\Sigma_{n}}=\hat{\mathcal{O}}_{n}\left(\prod_{I=1}^{n} Z_{G}\right)=\hat{U}_{\Sigma_{n}}\left(\prod_{i=1}^{2 n} Z_{K}^{a_{i}}\right)
$$

Here $\hat{\mathcal{O}}_{n}$ and $\hat{U}_{\Sigma_{n}}$ are certain intertwining operators, acting on the $t$-arguments of individual constituents and depending on the branch. For DV branches those operators simplify and become of Moyal-type.

This decomposition is exact matrix-model counterpart of instanton gas decomposition in YangMills theories: contribution of every saddle-point (i.e. a particular branch of partition function) can be decomposed into elementary constituents, associated with particular instantons [24] $\left(Z_{G}\right)$ and, further, merons [25, 26] $\left(Z_{K}\right)$. Parameters $a_{i}$ play the role of moduli of multi-instanton and multimeron configurations. The first step, decomposition of the DV solution into Gaussian (single-cut) partition functions $Z_{G}$ ("instantons") is straightforward, in what follows we concentrate mostly on the second step: decomposition of $Z_{G}$ into two $Z_{K}$ (of "instanton" into a pair of "merons"), which is more involved and less investigated (both at matrix-model and Yang-Mills levels).

\subsection{Partition function as a D-module}

Partition function $Z(t)$ of countable set of arguments $t_{0}, t_{1}, t_{2}, \ldots$ is defined [5, 1] as a $D$-module, i.e. as generic solution of the system of linear differential equations: ${ }^{1}$ the discrete Virasoro constraints [4, 27.

$$
\begin{gathered}
\hat{L}_{n} Z=0, \quad n \geq-1, \\
\hat{L}_{n}=\sum_{k>0} k t_{k} \frac{\partial}{\partial t_{k+n}}+g^{2} \sum_{a+b=n} \frac{\partial^{2}}{\partial t_{a} \partial t_{b}}
\end{gathered}
$$

Parameter $g$ could be absorbed into rescaling of $t$-variables, but it is used to select an important class of solutions (branches of $Z$ ), which "possess genus expansion" [28, i.e. such that

$$
g^{2} \log Z(t)=\sum_{p=0}^{\infty} g^{2 p} \mathcal{F}^{(p)}(t)
$$

\footnotetext{
${ }^{1}$ In such framework the usual matrix integral [3] is nothing but a particular integral representation of partition function. As usual with integral representations, different branches are associated with different choices of integration hypersurface in the space of matrices of arbitrary size.
} 
is a formal series in non-negative powers of $g^{2}$ with $g$-independent genus- $p$ prepotentials $\mathcal{F}^{(p)}(t)$. Such branches are further distinguished and labeled by hyperelliptic Riemann surfaces (spectral curves)

$$
\Sigma_{n}: \quad y^{2}=\prod_{i=1}^{2 n}\left(z-a_{i}\right)
$$

so that $\mathcal{F}^{(p)}\left(t^{\Sigma} \mid \Sigma_{n}\right)$ are formal series in non-negative powers of $t^{\Sigma}$ 's with $a_{i}$ 's allowed in denominators. The variables $t^{\Sigma}$ are related to original $t$ by $a$-dependent shifts:

$$
t_{k}=-T_{k}(a)+t_{k}^{\Sigma}, \quad T_{k}=0 \text { for } k>n+1
$$

See [1] and [13] for detailed description of this branch/phase structure of $Z(t)$. Below for the sake of brevity, we write all the partition functions as functions of $t$, not of $t^{\Sigma}$, since for all our calculations up to the Appendices it makes no difference (we need only commutation relations of these variables and the derivatives w.r.t. them). However, in the Appendices, where we obtain partition functions as formal series, we restore the notations $t^{\Sigma}$.

\subsection{Krichever-Novikov type algebra and the $\star$-product}

Virasoro operators $\hat{L}_{n}$ are components of the Virasoro stress-tensor: the loop operator

$$
\hat{L}(z)=\sum_{n=-\infty}^{+\infty} \frac{(d z)^{2}}{z^{n+2}} \hat{L}_{n}
$$

Virasoro constraint is the "negative" part of this two-form,

$$
\hat{L}_{-}(z)=\sum_{n=-1}^{+\infty} \frac{(d z)^{2}}{z^{n+2}} \hat{L}_{n}
$$

The language of loop operators has two advantages: it makes especially simple the Sugawara embedding of Virasoro operators into the universal enveloping of Kac-Moody algebra: $\hat{L}(z)=: \hat{J}^{2}(z)$ :, where

$$
\hat{J}(z)=\sum_{k \geq 0}\left\{\frac{1}{2} k t_{k} z^{k-1} d z+\frac{d z}{z^{k+1}} \frac{\partial}{\partial t_{k}}\right\}
$$

and it allows straightforward deformation to arbitrary Riemann surfaces (above formulas are essentially written on a sphere or, better, in the vicinity of a point $z=\infty$ ). Not-surprisingly, when we deal with a particular branch of partition function, the relevant deformation is to the spectral curve $\Sigma_{n}$ [17.

On a given Riemann surface (complex curve) $\Sigma$ the current $\hat{\mathbf{J}}(z)$ is an operator-valued analytical $(\partial \hat{\mathbf{J}} / \partial \bar{z}=0)$ 1-form, allowed to have singularities of a given type at certain points. Such $\hat{\mathbf{J}}(z)$ form a Krichever-Novikov-type algebra 29]-31. In the simplest case of the Riemann sphere $\Sigma=C P_{1}$ with two punctures, where $\hat{\mathbf{J}}(z)$ is allowed to have poles of arbitrary order, i.e. is an operated-valued Laurent series, it generates just an ordinary $\hat{U}(1)_{k=1}$ Kac-Moody algebra with the commutation relations

$$
\left[\hat{\mathbf{J}}^{C P_{1}}(z), \hat{\mathbf{J}}^{C P_{1}}\left(z^{\prime}\right)\right]=\frac{1}{2} \sum_{k=-\infty}^{\infty} \frac{k z^{k-1}}{z^{\prime k+1}} d z d z^{\prime}=-\frac{1}{2} \sum_{k=-\infty}^{\infty} \frac{k z^{k-1}}{z^{k+1}} d z d z^{\prime} \equiv \frac{1}{2} \delta^{\prime}\left(z-z^{\prime}\right) d z d z^{\prime}
$$

The sums at the r.h.s. define $z$-derivative of "holomorphic $\delta$-function", satisfying $\oint_{\infty} \delta\left(z-z^{\prime}\right) f\left(z^{\prime}\right)=$ $f(z)$ for arbitrary Laurent series $f(z)$.

Since matrix model is associated with Virasoro constraints, formed by the square of $\hat{J}(z)$, the corresponding $\hat{\mathbf{J}}(z)$ can have ramifications of degree two and are naturally defined on hyperelliptic Riemann surfaces. Of actual interest for us will be $\hat{\mathbf{J}}(z)$ on hyperelliptic $\Sigma_{n}: y^{2}=\prod_{i=1}^{2 n}\left(z-a_{i}\right)$ with singularities allowed at $z=a_{i}$ and at $z=\infty_{ \pm}$, which changes sign whenever $z$ goes around any of the points $a_{i}$, i.e. is expandable in formal series with odd powers of $\sqrt{z-a_{i}}$ near $z=a_{i}$ and in arbitrary 
Laurent series (with integer powers of $z^{-1}$ ) near $z=\infty_{ \pm}$(expansions at two infinities coincide). If all ramification points $a_{i}$ merge pairwise (all cuts are contracted to points), then the algebra reduces to that with poles only (no ramifications). Vice versa, sometime it can make sense to blow up the poles at infinities into $n$ additional cuts between $2 n$ new ramification points $\Lambda_{1}, \ldots, \Lambda_{2 n}$ [ 8 . We give further details about the loop algebras of this type below. Of principal importance will be emergency of conjugation operators, intertwining currents in different charts. They reveal the hidden symplectic (quantum) structure ( $\star$-product) of matrix model, which is important feature of generic string-field theory [32], of which the matrix model is a representative toy-example.

\subsection{Seiberg-Witten structure and the $*$-product}

The Virasoro-constraint operator is not quite $\hat{L}(z)=: \hat{J}^{2}(z):-$ it is rather $\hat{L}_{-}(z)$. The "minusprojection" is actually another structure added to Krichever-Novikov-type algebra. For $C P_{1}$ the minus-projection is just $f_{-}(z)=\oint_{0} \frac{f\left(z^{\prime}\right) d z^{\prime}}{z-z^{\prime}}=\oint_{\infty_{ \pm} ; z} \frac{f\left(z^{\prime}\right) d z^{\prime}}{z^{\prime}-z}$. However, since $\hat{L}(z)$ is a 2-differential, it can not be integrated along a contour without additional comments. In fact,

$$
\hat{\mathbf{L}}_{-}(z)=: \hat{\mathbf{J}} * \hat{\mathbf{J}}:(z)=\oint_{C} \mathcal{K}\left(z, z^{\prime}\right): \hat{\mathbf{J}}^{2}\left(z^{\prime}\right):
$$

where contour $C$ encircles $\infty_{ \pm}$and $z$ or, alternatively, all the ramification points $a_{i}$, and $\mathcal{K}\left(z, z^{\prime}\right)$ is a certain $(1,-1)$-bidifferential. Note that we define $\hat{\mathbf{L}}_{-}(z)$ as a 1 -form, not a 2 -differential(!), making use of peculiar $*$-product in the space of analytic 1-forms (see 33. for introductory discussion of such products). $\mathcal{K}\left(z, z^{\prime}\right)$ can be obtained from the readily available $(m, n)$-bidifferentials with $m, n \geq 0$ the free-field Green functions on Riemann surfaces [34] - by division over some 1-differential, which is the above-mentioned additional structure on $\Sigma_{n}$.

This structure is nothing but the celebrated Seiberg-Witten structure [35- 39], and its relevant version is the Dijkgraaf-Vafa differential [6] $\Omega_{D V}=y(z) d z$, so that ${ }^{2}$

$$
\mathcal{K}\left(z, z^{\prime}\right)=\frac{\left\langle\partial \phi(z) \phi\left(z^{\prime}\right)\right\rangle_{\Sigma_{n}}}{\Omega_{D V}\left(z^{\prime}\right)}
$$

An exact relation between the $\star$-structure behind the conjugation operators and the $*$-structure, their connection to Kontsevich quantization, Batalin-Vilkovissky and string-field-theory formalisms remain beyond the scope of the present paper. These subjects are relevant for discussion of cubic Eynard-type actions [12, 14] and the background-independence phenomenon.

\subsection{Local Virasoro constraints}

Operator $\hat{\mathbf{L}}_{-}(z)$ reduces to $\hat{L}_{-}(z)$, used in the definition of $Z(t)$, in the vicinity of $z=\infty$, and the basic identity

$$
\hat{L}_{-}^{G}(z) \sim \hat{\mathbf{L}}_{-}(z)=\oint_{\infty \cup z} \mathcal{K}\left(z, z^{\prime}\right): \hat{\mathbf{J}}^{2}\left(z^{\prime}\right):=-\sum_{i=1}^{2 n} \oint_{a_{i}} \mathcal{K}\left(z, z^{\prime}\right): \hat{\mathbf{J}}^{2}\left(z^{\prime}\right): \equiv-\sum_{i=1}^{2 n} \hat{\mathbf{L}}_{-}^{\left(a_{i}\right)}(z) \sim-
$$

implies that $Z(t)$ - the zero mode of $\hat{L}_{-}(z)$ - is a product of zero-modes of the commuting operators $\hat{\mathbf{L}}_{-}^{\left(a_{i}\right)}(z)$. Operators $\hat{\mathbf{L}}_{-}^{\left(a_{i}\right)}(z)$ are defined in the vicinities of particular ramification points, where $\hat{\mathbf{J}}(z)$ behaves as an odd current, therefore, up to a common conjugation, $\hat{\mathbf{L}}_{-}^{\left(a_{i}\right)}(z)$ are "continuous Virasoro constraints", which annihilate the Kontsevich $\tau$-function $Z_{K}\left(\tau_{k}^{\left(a_{i}\right)}\right)$ [40. The time-variables $\tau_{k}^{\left(a_{i}\right)}$ are introduced below.

\footnotetext{
${ }^{2}$ See [11, 12] For more details about this ratio of $d \mathcal{G}\left(z, z^{\prime}\right)$, the one differential w.r.t. the first argument and the primitive of the Bergmann kernel w.r.t. the second argument, and the Dijkgraaf-Vafa differential $\Omega_{D V}$. In simplest particular case of the Gaussian model, $\Sigma_{G}: y_{G}^{2}(z)=z^{2}-4 S$

$$
\mathcal{K}\left(z, z^{\prime}\right)=\frac{d z}{d z^{\prime}} \frac{1}{z-z^{\prime}}\left(\frac{1}{y_{G}(z)}-\frac{1}{y_{G}\left(z^{\prime}\right)}\right)
$$
}




\subsection{Generic construction. Summary}

Algebraic formalism described in this paper is easily expandable to zero-modes ( $D$-modules) of $W$ algebras, annihilated by projected powers of $\hat{J}(z)$-operators, not obligatory squares. For $\hat{W}^{(q)} \sim$ $\hat{J}^{q}$ spectral surfaces are not obligatory hyperelliptic. The relevant Seiberg-Witten structures and associated Whitham hierarchies, quasiclassical $\tau$-functions and WDVV equations deserve investigation.

We end our conceptual explanation of decomposition formulas with the following generic plan for their investigation:

\section{Currents}

- Riemann surface $\Sigma \rightarrow$ loop current algebra $\hat{\mathbf{J}}^{\Sigma}(z)$

- Global realization of the current algebra on $\Sigma$

- Its projections to standard realizations in vicinities of singularities

- Gaussian case: two poles at $\alpha$ and $\infty$ with $\alpha$ blown up into a cut between $a_{-}$and $a_{+}, a_{ \pm}=\alpha \pm \sqrt{S}$

\section{Sugawara realization of constraints}

- Generic Virasoro $(q=2)$ case: $n+1$ poles at $\alpha_{I}$ and $\infty$ with each $\alpha_{I}$ blown up into a cut between $a_{2 I-1}$ and $a_{2 I}, I=1, \ldots, n$

- $\hat{\mathbf{W}}^{(q)}(z)=\oint_{C} \mathcal{K}_{q}\left(z, z^{\prime}\right) \hat{\mathbf{J}}^{q}\left(z^{\prime}\right)$ and current is allowed to have ramification singularities of orders which are divisors of $q$

- The contour $C=C_{\infty}=\sum_{i}^{2 n} C_{i}$

\section{Partition functions}

- Partition function satisfies $\hat{\mathbf{W}}^{(q)}(z) \mathbf{Z}_{q}=\left(\oint_{C_{\infty}} \mathcal{K}_{q} \hat{\mathbf{J}}^{q}\right) \mathbf{Z}_{q}=0$

- Particular equations $\left\{\hat{U}^{-1}\left(\oint_{C_{i}} \mathcal{K}_{q} \hat{\mathbf{J}}^{q}\right) \hat{U}\right\} Z_{g K}^{(i)}=0$ with a common conjugation operator define the generalized Kontsevich $\tau$-functions $Z_{g K}$

- It follows that $\mathbf{Z}_{q}=\hat{U}\left(\prod_{i}^{2 n} Z_{g K}^{(i)}\right)$

\section{Shifts}

- Shifts of $t$-variables $t \rightarrow t^{\Sigma}$, normal ordering, background independence, representation of $Z_{q}$ and $Z_{g K}$ as formal series in $t^{\Sigma}$ variables.

The remaining part of this paper describes realization of this plan for $q=2$ in the simplest Gaussian (1-cut, $n=1$ ) case, thus completing the program, originally outlined in [17. On our way, we reproduce numerous particular results obtained during the last decade. The central decomposition formula in this case was originally obtained in [18] "by brute force". Certain controversy, persisted since the second paper of [19], is resolved below in s.7 (especially formula (7.17)). Moment variables and related simplifications of genus expansion of Kontsevich $\tau$-function were deeply investigated in [41, for moment variables in Hermitean matrix model see 42]. Group-theory approach and connections to topological theory were considered in [43, 44].

Our approach should be useful for study of decomposition formulas for different topological string theories. In particular, the basic example of the decomposition formula considered in this paper, corresponding to the simplest branch of the Hermitian matrix model, that is, to the Gaussian branch is in a sense dual to the simplest example of the Givental-style decomposition formula in topological strings. That formula is for the $\mathbb{C P}_{1}$ model, its partition function having a matrix model representation similar to the Gaussian matrix model, [45]. This partition function is known to be decomposed into a Moyal-like product of two Kontsevich $\tau$-function [46]. 


\section{Summary of the Gaussian example}

\subsection{Ingredients of the construction}

The starting point in this example is the Gaussian curve

$$
\Sigma_{G}: \quad y_{G}^{2}=\left(z-a_{+}\right)\left(z-a_{-}\right)
$$

This is the Riemann sphere in hyperelliptic parametrization with two quadratic ramification points $a_{ \pm}$and two infinities $\infty_{ \pm}$. Throughout the paper, we often (but not always) put $a_{-}=-a_{+}=-2 \sqrt{S}$. Since this is the sphere, there is a globally defined coordinate $w$,

$$
z=\sqrt{S}\left(w+\frac{1}{w}\right), \quad d z=\sqrt{S}\left(1-\frac{1}{w^{2}}\right) d w, \quad y_{G}(z)=\sqrt{S}\left(w-\frac{1}{w}\right)
$$

The second ingredient is the current $\hat{\mathbf{J}}$, allowed to have singularities at these four punctures,

$$
\begin{gathered}
\hat{\mathbf{J}}=\hat{\mathbf{J}}^{e}\left(z, y_{G}\right)+\hat{\mathbf{J}}_{G}^{o}\left(z, y_{G}\right) \\
\hat{\mathbf{J}}^{e}\left(z, y_{G} \mid R, Q\right) \equiv \sum_{k=0}^{\infty}\left\{\frac{1}{2}\left(k+\frac{1}{2}\right)\left(R_{k}+z Q_{k}\right) y_{G}^{2 k} d z+g^{2} \frac{d z}{y_{G}^{2 k+2}}\left(\frac{\partial}{\partial \tilde{R}_{k}}+z \frac{\partial}{\partial \tilde{Q}_{k}}\right)\right\} \\
\hat{\mathbf{J}}^{o}\left(z, y_{G} \mid T, S\right) \equiv \sum_{k=0}^{\infty}\left\{\frac{1}{2}\left(k+\frac{1}{2}\right)\left(T_{k}+z S_{k}\right) y_{G}^{2 k-1} d z+g^{2} \frac{d z}{y_{G}^{2 k+3}}\left(\frac{\partial}{\partial \tilde{T}_{k}}+z \frac{\partial}{\partial \tilde{S}_{k}}\right)\right\}
\end{gathered}
$$

where we separate even and odd parts of the current w.r.t. the $\mathbb{Z}_{2}$-symmetry $y_{G} \rightarrow-y_{G}$ of $\Sigma_{G}$. Here the convenient parameter (coupling constant) $g$ is introduced by appropriate rescaling of timevariables, and commutation relations for the current require that

$$
\frac{\partial}{\partial \tilde{T}_{k}}=2\left(4 S \frac{\partial}{\partial T_{k}}+\frac{k+1}{k+\frac{3}{2}} \frac{\partial}{\partial T_{k+1}}\right), \quad \frac{\partial}{\partial \tilde{S}_{k}}=2 \frac{\partial}{\partial S_{k}}
$$

Indeed, the operator product expansions of the global currents (2.3) are

$$
\begin{gathered}
\hat{\mathbf{J}}^{e}(z) \hat{\mathbf{J}}^{e}\left(z^{\prime}\right)=g^{2} \frac{d z d z^{\prime}}{2\left(z-z^{\prime}\right)^{2}}+\text { regular part } \\
\hat{\mathbf{J}}^{o}(z) \hat{\mathbf{J}}^{o}\left(z^{\prime}\right)=g^{2} \frac{d z d z^{\prime}}{4\left(z-z^{\prime}\right)^{2}} \frac{2 z z^{\prime}-\left(a_{+}+a_{-}\right)\left(z+z^{\prime}\right)+2 a_{+} a_{-}}{y(z) y\left(z^{\prime}\right)}+\text { regular part }
\end{gathered}
$$

These formulas are consistent with the standard $U(1)$ Kac-Moody algebra. In order to check it, one suffices to make a change of coordinates to the globally defined variable $w$, (2.2) and to see that

$$
\begin{gathered}
\hat{\mathbf{J}}(z) \hat{\mathbf{J}}\left(z^{\prime}\right)=\hat{\mathbf{J}}^{e}(z) \hat{\mathbf{J}}^{e}\left(z^{\prime}\right)+\hat{\mathbf{J}}^{o}(z) \hat{\mathbf{J}}^{o}\left(z^{\prime}\right)+\text { regular part }= \\
=g^{2} \frac{d z d z^{\prime}}{4\left(z-z^{\prime}\right)^{2}}\left(\frac{2 z z^{\prime}-\left(a_{+}+a_{-}\right)\left(z+z^{\prime}\right)+2 a_{+} a_{-}}{y(z) y\left(z^{\prime}\right)}+2\right)+\text { regular part }=g^{2} \frac{d w d w^{\prime}}{2\left(w-w^{\prime}\right)^{2}}+\text { regular part }
\end{gathered}
$$

The variables in (2.3) are chosen in a way convenient for the symmetric model, i.e. that with $a_{+}=-a_{-}$. In the asymmetric case, one better puts

$$
\begin{aligned}
\hat{\mathbf{J}}^{o}\left(z, y_{G} \mid T^{ \pm}\right) & =\sum_{k=0}^{\infty}\left\{\frac{1}{2}\left(k+\frac{1}{2}\right)\left(T_{k}^{+}\left(z-a_{+}\right)+T_{k}^{-}\left(z-a_{-}\right)\right) y_{G}^{2 k-1} d z+\right. \\
& \left.+g^{2} \frac{d z}{y_{G}^{2 k+3}}\left(\left(z-a_{+}\right) \frac{\partial}{\partial \tilde{T}_{k}^{+}}+\left(z-a_{-}\right) \frac{\partial}{\partial \tilde{T}_{k}^{-}}\right)\right\},
\end{aligned}
$$

etc. 
The remaining ingredient is the $D$-module: the set of Virasoro constraints, e.g.,

$$
\hat{\mathbf{L}}_{-}^{o}(z)=: \hat{\mathbf{J}}^{o} * \hat{\mathbf{J}}^{o}:(z)=\oint_{C} \mathcal{K}_{G}\left(z, z^{\prime}\right):\left(\hat{\mathbf{J}}^{o}(z)\right)^{2}:
$$

and its solution (zero mode) - the Gaussian global partition function $\mathbf{Z}(T, S)$, - satisfying

$$
\hat{\mathbf{L}}_{-}^{o}(z) \mathbf{Z}(T, S)=0
$$

One may restrict the even and odd global currents in (2.3) further, making use of another $\mathbb{Z}_{2^{-}}$ symmetry of $\Sigma_{G}, z \rightarrow-z+a_{+}+a_{-}$( or $z \rightarrow-z$ in the symmetric model with $a_{+}=-_{-}$). This transformation exchanges, say, $T_{+}$and $T_{-}$in (2.7) and allows one to construct global partition functions like $\mathbf{Z}\left(T_{+}+T_{-}\right)$etc (see table 1$)$.

\subsection{Familiar special functions}

$\hat{\mathbf{L}}_{-}(z)$ and $\mathbf{Z}\left(T^{ \pm}\right)$are new personages in the theory. As explained in the Introduction, they interpolate between a variety of well known quantities and provide non-linear relations among them. In this paper we consider, in addition to $\mathbf{Z}$, three such familiar functions:

- The Gaussian branch $Z_{G}(t)$ of the Hermitean partition function [1, 13, annihilated by the "discrete Virasoro constraints" 27,

$$
\begin{gathered}
\hat{L}_{-}^{G}(z) Z_{G}(t)=0 \\
\hat{L}_{-}^{G}(z)=\left(: \hat{J}_{G}^{2}(z):\right)_{-}=g^{2} \sum_{n=-1}^{+\infty} \frac{(d z)^{2}}{z^{n+2}}\left(\sum_{k>0} k t_{k} \frac{\partial}{\partial t_{k+n}}+g^{2} \sum_{a+b=n} \frac{\partial^{2}}{\partial t_{a} \partial t_{b}}\right) \\
\hat{J}_{G}(z \mid t)=d \hat{\Omega}_{G}(z)=\frac{1}{2} d v(z)+g^{2} \hat{\nabla}(z)=\sum_{k=0}^{\infty}\left\{\frac{1}{2} k t_{k} z^{k-1} d z+g^{2} \frac{d z}{z^{k+1}} \frac{\partial}{\partial t_{k}}\right\}
\end{gathered}
$$

This partition function $Z_{G}$ has the Hermitean matrix integral representation

$$
Z_{G}=\frac{1}{\operatorname{Vol}_{U(N)}} \int D \phi \exp \left(\frac{1}{g} \sum_{k \in Z_{+}} t_{k} \operatorname{Tr} \phi^{k}\right)
$$

where measure is the invariant measure on Hermitean matrices of the size $N \times N$, the volume of the unitary group $U(N)$

$$
\mathrm{Vol}_{U(N)}=\int_{U(N)} D U \sim \prod_{k=1}^{N-1} k !
$$

and the integral (2.11) is understood as a perturbative power series in $t_{k}^{\Sigma}$ s, $t_{k}=t_{k}^{\Sigma}-\frac{1}{2} \delta_{k, 2}$.

Note that $\frac{\partial}{\partial t_{0}}$-term in the current $\hat{J}_{G}$ commutes with the whole current (there is no $t_{0}$ in the current) and, therefore, one may deal with $\frac{\partial}{\partial t_{0}}$ as with $c$-number. We, indeed, throughout the paper put $\frac{\partial Z_{G}}{\partial t_{0}}=\frac{S}{g^{2}} Z_{G}$. In particular, in (2.11) $S$ is associated with the size of matrix $N$. A generic case is discussed in s.6.2.

Other branches of $Z_{G}$, including the Dijkgraaf-Vafa ones and generic branches with genus expansion [1] can be also treated by the method of this paper. However, this will be done elsewhere. 
- Kontsevich $\tau$-function 20, 21] $Z_{K}(\tau)$, annihilated by "continuous Virasoro constraints" 40,

$$
\begin{gathered}
\hat{L}_{-}^{K}(\xi) Z_{K}(\tau)=0, \\
\hat{L}_{-}^{K}(\xi)=\left(: \hat{J}_{K}^{2}(\xi):\right)_{-}= \\
=g^{2} \sum_{n=-1}^{+\infty} \frac{(d \xi)^{2}}{\xi^{2 n+2}}\left(\sum_{k>0}\left(k+\frac{1}{2}\right) \tau_{k} \frac{\partial}{\partial \tau_{k+n}}+g^{2} \sum_{a+b=n-1} \frac{\partial^{2}}{\partial \tau_{a} \partial \tau_{b}}+\frac{\delta_{n, 0}}{16}+\frac{\delta_{n,-1} \tau_{0}^{2}}{16 g^{2}}\right) \\
\hat{J}_{K}(\xi \mid \tau)=d \hat{\Omega}_{K}(\xi)=\sum_{k=0}^{\infty}\left\{\frac{1}{2}\left(k+\frac{1}{2}\right) \tau_{k} \xi^{2 k} d \xi+g^{2} \frac{d \xi}{\xi^{2 k+2}} \frac{\partial}{\partial \tau_{k}}\right\}
\end{gathered}
$$

This partition function $Z_{K}$ can be presented as the Hermitean matrix integral depending on the external matrix $A$,

$$
Z_{K}=\frac{\int D X \exp \left(-\frac{4 g^{2}}{3} \operatorname{Tr} X^{3}-\frac{2 g}{\sqrt{3}} \operatorname{Tr} A X^{2}\right)}{\int D X \exp \left(-\frac{2 g}{\sqrt{3}} \operatorname{Tr} A X^{2}\right)}
$$

where the integral is understood as a perturbative power series in $\tau_{k}^{\Sigma} \equiv g \frac{3^{k+\frac{1}{2}}}{k+\frac{1}{2}} \operatorname{Tr} A^{-2 k-1}, \tau_{k}=$ $\tau_{k}^{\Sigma}-\frac{2}{3} \delta_{k, 1}$. Note that this integral does not depend on the size of matrices $X$ and $A$ being considered as a function of $\tau_{k}^{\Sigma}$ [21]. By the shift of the integration variable, it can be also reduced to the form

$$
Z_{K}=\exp \left(-\frac{1}{3 g} \operatorname{Tr} \Lambda^{\frac{3}{2}}\right) \frac{\int D X \exp \left(-\frac{4 g^{2}}{3} \operatorname{Tr} X^{3}+\operatorname{Tr} \Lambda X\right)}{\int D X \exp \left(-\frac{2 g}{\sqrt{3}} \operatorname{Tr} A X^{2}\right)}
$$

where $3 \Lambda=A^{2}$, i.e. $\tau_{k}^{\Sigma} \equiv \frac{g}{k+\frac{1}{2}} \operatorname{Tr} \Lambda^{-k-\frac{1}{2}} \cdot 3$

- Partition function of the complex matrix model [47, annihilated by the truncated "discrete Virasoro constraints" [17]

$$
\begin{gathered}
\hat{L}_{-}^{C}(z) Z_{C}(\mathfrak{t})=0 \\
\hat{L}_{-}^{C}(z)=\left(: \hat{J}_{C}^{2}(z):\right)_{-}=g^{2} \sum_{n=0}^{+\infty} \frac{(d z)^{2}}{z^{2 n+2}}\left(\sum_{k>0} k \mathfrak{t}_{k} \frac{\partial}{\partial \mathfrak{t}_{k+n}}+g^{2} \sum_{a+b=n} \frac{\partial^{2}}{\partial \mathfrak{t}_{a} \partial \mathfrak{t}_{b}}\right) \\
\hat{J}_{C}(z \mid \mathfrak{t})=\sum_{k=0}^{\infty}\left\{\frac{1}{2} k \mathfrak{t}_{k} z^{2 k-1} d z+g^{2} \frac{d z}{z^{2 k+1}} \frac{\partial}{\partial \mathfrak{t}_{k}}\right\}
\end{gathered}
$$

This partition function $Z_{C}$ has the complex matrix integral representation

$$
Z_{C}=\frac{1}{\operatorname{Vol}_{U(N)}^{2}} \int D \phi D \phi^{\dagger} \exp \left(\frac{1}{g} \sum_{k \in Z_{+}} \mathfrak{t}_{k} \operatorname{Tr}\left(\phi \phi^{\dagger}\right)^{k}\right)
$$

where the integral which goes over $N \times N$ complex matrices is understood as a perturbative power series in $\mathfrak{t}_{k}^{\Sigma}$ 's, $\mathfrak{t}_{k}=\mathfrak{t}_{k}^{\Sigma}-\delta_{k, 1}$. Similarly to the Hermitean case (2.10), we put $\frac{\partial Z_{C}}{\partial t_{0}}=\frac{S}{g^{2}} Z_{C}$.

\footnotetext{
${ }^{3}$ In order to introduce an arbitrary shifted first time, $\tau_{k}=\tau_{k}^{\Sigma}-\frac{2 M}{3} \delta_{k, 1}$, where $M$ is a parameter, one should consider instead of 2.14 the integral

$$
Z_{K}=\frac{\int D X \exp \left(-\frac{4 g^{2}}{3 M^{2}} \operatorname{Tr} X^{3}-\frac{2 g}{\sqrt{3 M}} \operatorname{Tr} A X^{2}\right)}{\int D X \exp \left(-\frac{2 g}{\sqrt{3 M}} \operatorname{Tr} A X^{2}\right)}=\exp \left(-\frac{M}{3 g} \operatorname{Tr} \Lambda^{\frac{3}{2}}\right) \frac{\int D X \exp \left(-\frac{4 g^{2}}{3 M^{2}} \operatorname{Tr} X^{3}+\operatorname{Tr} \Lambda X\right)}{\int D X \exp \left(-\frac{2 g}{\sqrt{3 M}} \operatorname{Tr} A X^{2}\right)}
$$

which is a function of the same $\tau_{k}^{\Sigma}=g \frac{3^{k+\frac{1}{2}}}{k+\frac{1}{2}} \operatorname{Tr} A^{-2 k-1}=\frac{g}{k+\frac{1}{2}} \operatorname{Tr} \Lambda^{-k-\frac{1}{2}}$.
} 
- To deal with decomposition formula for the complex matrix model, we will also need a partition function $\tilde{Z}_{K}(\mathcal{T})$ that solves the Virasoro constraints (2.13) only with $n \geq 0$ and with the zeroth time shifted. At the moment, we have no formula for its matrix model representation available, therefore, it can be only recurrently calculated from the Virasoro constraints.

In applications, first terms of the $\left(g^{2}, t\right)$-expansions of the partition functions are often needed, see Appendix I.

\subsection{Web of dualities between matrix models}

\begin{tabular}{|c|c|c|c|c|}
\hline $\begin{array}{l}\text { Type } \\
\text { of } \mathbf{Z}\end{array}$ & $\begin{array}{l}\text { Reduction } \\
\text { of } \mathbf{Z}\end{array}$ & $\begin{array}{l}\text { Global } \\
\text { current }\end{array}$ & $\begin{array}{c}\text { Vicinity of } \\
\infty_{ \pm}\end{array}$ & $\begin{array}{c}\text { Vicinity of } \\
a_{ \pm}\end{array}$ \\
\hline $\mathbf{Z}(T, S)$ & $\begin{array}{c}\mathbb{Z}_{2 \text {-odd }} \\
(y \rightarrow-y)\end{array}$ & $\hat{\mathbf{J}}^{o}(z)$ & $Z_{G}(t)$ & $Z_{K}\left(\tau_{ \pm}\right)$ \\
\hline $\mathbf{Z}(T)$ & $\begin{array}{l}\left.\mathbb{Z}_{2} \times \mathbb{Z}_{2} \text {-(odd,odd }\right) \\
(z \rightarrow-z, y \rightarrow-y)\end{array}$ & $\frac{\hat{\mathbf{J}}^{o}(z)-\hat{\mathbf{J}}^{o}(-z)}{2}$ & $Z_{C}\left( \pm t^{e}\right)$ & $Z_{K}\left(\tau_{ \pm}\right)$ \\
\hline $\mathbf{Z}(S)$ & $\begin{array}{r}\left.\mathbb{Z}_{2} \times \mathbb{Z}_{2} \text {-(even,odd }\right) \\
(z \rightarrow-z, y \rightarrow-y)\end{array}$ & $\frac{\hat{\mathbf{J}}^{o}(z)+\hat{\mathbf{J}}^{o}(-z)}{2}$ & $Z_{K}\left( \pm t^{o}\right)$ & $Z_{K}\left(\tau_{ \pm}\right)$ \\
\hline $\mathbf{Z}(R, Q)$ & $\begin{array}{l}\mathbb{Z}_{2} \text {-even } \\
(y \rightarrow-y)\end{array}$ & $\hat{\mathbf{J}}^{e}(z)$ & $Z_{G}(t)$ & $Z_{C}\left(\tau_{ \pm}\right)$ \\
\hline $\mathbf{Z}(R)$ & $\begin{array}{r}\mathbb{Z}_{2} \times \mathbb{Z}_{2^{-}}(\text {odd, even }) \\
(z \rightarrow-z, y \rightarrow-y)\end{array}$ & $\frac{\hat{\mathbf{J}}^{e}(z)-\hat{\mathbf{J}}^{e}(-z)}{2}$ & $Z_{K}\left(t^{o}\right)$ & $Z_{C}\left(\tau_{ \pm}\right)$ \\
\hline $\mathbf{Z}(Q)$ & $\begin{array}{c}\left.\mathbb{Z}_{2} \times \mathbb{Z}_{2^{-}} \text {(even,even }\right) \\
(z \rightarrow-z, y \rightarrow-y)\end{array}$ & $\frac{\hat{\mathbf{J}}^{e}(z)+\hat{\mathbf{J}}^{e}(-z)}{2}$ & $Z_{C}\left(t^{e}\right)$ & $Z_{C}\left(\tau_{ \pm}\right)$ \\
\hline
\end{tabular}

Table 1. Relations between global currents and partition functions, their symmetry and partition functions emerging at singularities 
In the vicinities of punctures $\infty_{ \pm}$and $a_{ \pm}$the global current $\hat{\mathbf{J}}$ (or its reductions to $\hat{\mathbf{J}}^{o}$ etc) is isomorphic to $\hat{J}_{G}^{\infty}{ }^{\infty}$ (whose reductions gives rise to $\hat{J}$ etc) and $\hat{J}^{a \pm}$ (whose reductions gives rise to $\hat{J}_{K}$ etc) respectively. Isomorphism is realized by certain conjugation operators, depending on the choice of local coordinates and associated relation between the time-variables. Accordingly, the above special functions, which are the zero-modes of the thus related Virasoro constraints, are expressed by action of these operators on $\mathbf{Z}$. Moreover, one could start with reduced global currents, say, with $\mathbf{J}^{o}$ and consider its projections to the local patches, which also generates proper projections of the partition functions.

In the table above, we consider these projections of various global partition functions to the local partition functions in vicinities of the four singular points. Those local partition functions are the Hermitean matrix model, $Z_{G}$, the complex matrix model, $Z_{C}$ and the Kontsevich matrix model, $Z_{K}$. In the table, we denote through $t^{o}$ the odd set $\left\{t_{2 k+1}\right\}$ and through $t^{e}$ the even set $\left\{t_{2 k+1}\right\}$ of variables $\left\{t_{k}\right\}$. The table is fully formulated in terms of the symmetric currents (2.3), though (2.7) is needed at intermediate steps in some derivations.

In order to illustrate our general construction, we also consider below an example of the sphere with two more singular points placed in $z=0$ on the both sheets. In this case, we deal with the global partition function of the type $\mathbf{Z}(T)$, it has as its local projection in the vicinity of the new singular point the partition function $\tilde{Z}(\mathcal{T})$.

\subsection{Moment variables}

In fact, we are going to add to the list of special functions three more that are associated with the different set of variables, moment variables, defined so that, in these variables, the finite genus contribution to the partition function $\mathcal{F}^{(p)}$ is a polynomial. (Instead, discrete and continuous Virasoro constraints lose their simple form in these coordinates.) Besides, these variables are graded and every finite genus contribution involves only a few first moment variables, their number increasing with genus (the degree of gradation grows with genus). Therefore, the moments, first, depend on the concrete genus expansion and, second, admit arbitrary triangle change of variables, which does not change their defining property).

\subsubsection{Changing local parameter}

The simplest way to introduce moment variables is to consider changing local parameters in vicinities of the four singular points. This way one introduce two new sets of variables:

- Moments for the Kontsevich $\tau$-function

$$
\tilde{\tau}_{m}=G^{-\frac{2 m+1}{3}} \sum_{k=0}^{\infty} \frac{\Gamma(m+k+3 / 2) u^{k}}{\Gamma(m+3 / 2) k !} \tau_{m+k},
$$

corresponding to the change of the local parameter

$$
\tilde{\xi}=G^{1 / 3} \sqrt{\xi^{2}-u}
$$

where $u$ and $G$ are some specific functions of $\tau_{k}$ fixed by the conditions

$$
\tilde{\tau}_{0}^{\Sigma}=\tilde{\tau}_{1}^{\Sigma}=0
$$

Note that the shift in this case is the same in both $\tau$ and $\tilde{\tau}$ variables,

$$
\tau_{m} \equiv \tau_{m}^{\Sigma}-\frac{2}{3} \delta_{m, 1}, \quad \tilde{\tau}_{m} \equiv \tilde{\tau}_{m}^{\Sigma}-\frac{2}{3} \delta_{m, 1}
$$


- Moments for the Gaussian branch of $Z_{G}$

$$
m \tilde{t}_{m}=\oint_{\infty} \frac{d v(z)}{\tilde{z}^{m}}=a \oint_{\infty} \frac{\sum_{k} k t_{k}(a z+b)^{n-1} d z}{z^{m}}=a^{m} \sum_{k} \frac{k ! b^{k-m}}{(m-1) !(k-m) !} t_{k}
$$

that correspond to the change of the local parameter

$$
\tilde{z}=A \tilde{z}+B
$$

where $A, B$ are functions of $t_{k}$ fixed by the conditions

$$
\tilde{t}_{1}^{\Sigma}=\tilde{t}_{2}^{\Sigma}=0
$$

Again the shift is the same in both $t$ and $\tilde{t}$ variables,

$$
t_{k}=t_{k}^{\Sigma}-\frac{\delta_{k, 2}}{2}, \quad \tilde{t}_{k}=\tilde{t}_{k}^{\Sigma}-\frac{\delta_{k, 2}}{2}
$$

While the Kontsevich moments correspond to the standard genus expansion of $Z_{K}$, the moment variables for $Z_{G}$ are associated with a non-standard expansion, see s.2.5.1.

\subsubsection{Moment variables for the standard genus expansion of $Z_{G}$}

One can also express $Z_{G}$ it terms of moments that provide the standard genus expansion for it [42],

$$
t_{k}^{ \pm}=\oint_{A_{-}, A_{+}} \frac{v(z) d z}{\left(z-A_{\mp}(t)\right)^{k} y_{G}(z)}, \quad v(z)=\sum t_{k} z^{k}, \quad y_{G}(z) \equiv\left(z-A_{+}(t)\right)\left(z-A_{-}(t)\right)
$$

The simplest way then to calculate the corresponding partition function,

$$
Z_{A C K M}\left(t^{ \pm}\right) \equiv Z_{G}(t)
$$

is to use the relation (see s.2.7 and s.7) between $Z_{G}(t)$ and $Z_{K}\left(\tau^{ \pm}\right)$parameterized by an arbitrary branching points $a_{ \pm}$, to rescale $Z_{K}\left(\tau^{ \pm}\right)$and time variables $\tau^{ \pm} \rightarrow \tilde{\tau}^{ \pm}$, and, at the final stage of the calculation, to make ${ }^{4} a_{ \pm}=A_{ \pm}(\tau)$ depending on $\tau^{ \pm}$in such a way that $\tau_{0}^{ \pm}=\tau_{1}^{ \pm}=0 .{ }^{5}$ This can be formulated as a kind of normal ordering: one can consider, by definition,

$$
: e^{\hat{\tilde{U}}_{G K}} Z_{K}\left(\tilde{\tau}_{+}\right) Z_{K}\left(\tilde{\tau}_{-}\right):
$$

as being calculated at constant $a_{ \pm}=A_{ \pm}(\tau)$.

\subsection{Partition functions: genus and polynomial expansions}

In order to illuminate the role of moment variables, let us discuss structure of the partition functions as power series in $g$ and $t^{\Sigma}$.

\subsection{1 $Z_{G}$}

Let us assume existence of the genus expansion,

$$
\log Z_{G}(t)=\sum_{p=0}^{\infty} g^{2 p-2} F_{N}^{(p)}(t)
$$

\footnotetext{
${ }^{4}$ We use capital $A$ in order to stress that it becomes a function of times.

${ }^{5}$ The idea of this calculation has first appeared in the improved version of [19].
} 
The index $N$ stresses that the free energy also depends on $N$. Now we note that the Virasoro constraints (2.10) are invariant under simultaneous rescalings

$$
t_{k} \rightarrow \lambda^{k+s} t_{k}, \quad M \rightarrow \lambda^{2+s} M, \quad g \rightarrow \lambda^{s} g
$$

with arbitrary $s$. Here we introduced a parameter $M$ that controls the shift of times, $t_{k}=t_{k}^{\Sigma}-\frac{M}{2} \delta_{k, 2}$. Since we look for the partition function as a power series in $g$ and $t^{\Sigma}, F_{N}^{(p)}\left(\lambda^{2+s} M, \lambda^{k+s} t_{k}\right)=$ $\lambda^{2 s(1-p)} F_{N}^{(p)}\left(M, t_{k}\right)$, and

$$
F_{N}^{(p)}(M, t)=\sum_{r} \sum_{k_{1}, \ldots, k_{r}} C_{k_{1} \ldots k_{r}}^{(p)} \frac{t_{k_{1}}^{\Sigma} \ldots t_{k_{r}}^{\Sigma}}{M^{\frac{1}{2}\left(k_{1}+\ldots+k_{r}\right)}} \delta\left(\sum_{i=1}^{r}\left(k_{i}-2\right)-4(p-1)\right)
$$

The topological correlators $C_{k_{1} \ldots k_{r}}^{(p)}$ can be found from recurrent relations emerging from (2.10) upon inserting there the anzatz (2.32).

Selection rule, expressed by the $\delta$-factor in (2.32), implies that for $t_{1}^{\Sigma}=t_{2}^{\Sigma}=0$, the spherical and toric free energies $F_{N}^{(0)}-N t_{0} / g=F_{N}^{(1)}=0$ and all higher $F_{N}^{(p)}$ with $p \geq 2$ are polynomials of finite ( $p$-dependent) degree in remaining time-variables $t_{k}^{\Sigma}, k \geq 3$. This is exactly what moment variables provide: using variables (2.23) and fixing specific time-dependent $a(t)$ and $b(t)$ in their definition, one comes to $Z_{G}(\tilde{t})$ with $\tilde{t}_{1}^{\Sigma}=\tilde{t}_{2}^{\Sigma}=0$, i.e. to the polynomial representations of $F_{N}^{(p)}\left(\tilde{t}^{\Sigma}\right)$ 's.

These moment variables have only one drawback - they are associated with the genus expansion that is done at constant $N$, while the standard matrix model genus expansion is done at constant $S=g N$. This another expansion immediately destroys the simple scheme above and forces one to use more tricky moment variables in order to get a polynomial representation for the standardly genus expanded free energy, see s.2.4.2 and s.7.

\subsection{2 $Z_{K}$}

Similarly to the case of $Z_{G}$, we expand $Z_{K}$ into power series in $g$ and $\tau^{\Sigma}$,

$$
\log Z_{K}(\tau)=\sum_{p=0}^{\infty} g^{2 p-2} \mathcal{F}^{(p)}(\tau), \quad \tau=\tau^{\Sigma}-\frac{2 M}{3} \delta_{k, 1}
$$

Again note that the Virasoro constraints (2.13) are invariant under simultaneous rescalings

$$
\tau_{k} \rightarrow \lambda^{2 k+1+s} \tau_{k}, \quad M \rightarrow \lambda^{3+s} M, \quad g \rightarrow \lambda^{s} g
$$

with arbitrary $s$. This means that $\mathcal{F}^{(p)}\left(\lambda^{3+s} M, \lambda^{2 k+1+s} \tau_{k}\right)=\lambda^{2 s(1-p)} \mathcal{F}^{(p)}\left(M, \tau_{k}\right)$, and

$$
\mathcal{F}^{(p)}(M, \tau)=\sum_{r} \sum_{k_{1}, \ldots, k_{r}} \mathcal{C}_{k_{1} \ldots k_{r}}^{(p)} \frac{\tau_{k_{1}}^{\Sigma} \ldots \tau_{k_{r}}^{\Sigma}}{M^{\frac{r}{3}+\frac{2}{3}\left(k_{1}+\ldots+k_{r}\right)}} \delta\left(\sum_{i=1}^{r}\left(k_{i}-1\right)-3(p-1)\right)
$$

and topological correlators $\mathcal{C}_{k_{1} \ldots k_{r}}^{(p)}$ can be found from recurrent relations emerging from (2.13) upon inserting there the anzatz (2.35).

This time the selection rule, expressed by the $\delta$-factor in (2.35), implies that for $\tau_{0}^{\Sigma}=\tau_{1}^{\Sigma}=0$, the spherical and toric free energies $\mathcal{F}^{(0)}=\mathcal{F}^{(1)}=0$ and all higher $\mathcal{F}^{(p)}$ with $p \geq 2$ are polynomials of finite ( $p$-dependent) degree in remaining time-variables $\tau_{k}^{\Sigma}, k \geq 2$.

Again, this is what moment variables provide: using variables (2.19) and fixing specific timedependent $G(t)$ and $u(t)$ in their definition, one comes to $Z_{K}\left(\tilde{\tau}^{\Sigma}\right)$ with $\tilde{\tau}_{1}^{\Sigma}=\tilde{\tau}_{2}^{\Sigma}=0$, i.e. to the polynomial representations of $\mathcal{F}^{(p)}\left(\tilde{\tau}^{\Sigma}\right)$ 's. 


\subsubsection{Non-standard genus expansions}

One can also use another non-standard genus expansion for $Z_{K}$ which allows one to deal with polynomials at each order in the standard variables without making the special change of variables to $\tilde{\tau}_{0}^{\Sigma}=\tilde{\tau}_{1}^{\Sigma}=0$. To this end, one suffices to rescale the time variables $\tau_{k}^{\Sigma}=g \tau_{k}^{\prime \Sigma}$. Then,

$$
\log Z_{K}\left(\tau^{\Sigma}\right)=\sum_{p=0}^{\infty} g^{2 p-2} \mathcal{F}_{(p)}^{\prime}\left({\tau^{\prime}}^{\Sigma}\right)
$$

Then, indeed, the only non-polynomial part of the genus $g$ free energy, that is, that containing power series in $\tau_{0}^{\prime \Sigma}$ and $\tau_{1}^{\prime \Sigma},(2.35)$ becomes a polynomial.

The similar change of variables in $Z_{G}, t_{k}=g t^{\prime \Sigma}$ gives rise to the genus expansion

$$
\log Z_{G}\left(t^{\Sigma}\right)=\sum_{p=0}^{\infty} g^{2 p-2} F_{(p)}^{\prime}\left(t^{\prime^{\Sigma}}\right)
$$

Let us now represent [1]

$$
F^{(p)}\left(t^{\Sigma}\right)=\sum_{k=1}^{\infty} \frac{1}{k !} \oint \ldots \oint \rho^{(p \mid k)}\left(z_{1}, \ldots, z_{k} \mid S\right) v\left(z_{1}\right) \ldots v\left(z_{k}\right)
$$

with some coefficient functions $\rho^{(p \mid k)}\left(z_{1}, \ldots, z_{k} \mid S\right)$ (which are $k$-point resolvents, or loop operators). From this expansion, it is clear that $F_{(1)}^{\prime}$ is linear in (infinitely many) times, $F_{(2)}^{\prime}$ is quadratic, $F_{(3)}^{\prime}$ gets contributions from $F_{(3)}$ and $F_{(1)}$ and contains both linear and cubic terms etc:

$$
\begin{gathered}
F_{(1)}\left(t^{\prime}\right)=\oint \rho^{(0 \mid 1)}(z) v^{\prime}(z) \\
F_{(2)}\left(t^{\prime}\right)=\frac{1}{2 !} \oint \rho^{(0 \mid 2)}\left(z_{1}, z_{2}\right) v^{\prime}\left(z_{1}\right) v^{\prime}\left(z_{2}\right) \\
F_{(3)}\left(t^{\prime}\right)=\frac{1}{3 !} \oint \rho^{(0 \mid 3)}\left(z_{1}, z_{2}, z_{3}\right) v^{\prime}\left(z_{1}\right) v^{\prime}\left(z_{2}\right) v^{\prime}\left(z_{3}\right)+\oint \rho^{(1 \mid 1)}(z) v^{\prime}(z) \\
F_{(4)}\left(t^{\prime}\right)=\frac{1}{4 !} \oint \rho^{(0 \mid 4)}\left(z_{1}, z_{2}, z_{3}, z_{4}\right) v^{\prime}\left(z_{1}\right) v^{\prime}\left(z_{2}\right) v^{\prime}\left(z_{3}\right) v^{\prime}\left(z_{4}\right)+\frac{1}{2 !} \oint \rho^{(1 \mid 2)}\left(z_{1}, z_{2}\right) v^{\prime}\left(z_{1}\right) v^{\prime}\left(z_{2}\right)
\end{gathered}
$$

where $v^{\prime}(z)$ denotes $\sum t_{k}^{\prime} z^{k}$.

These polynomials $F_{(k)}\left(t^{\Sigma}\right)$ containing infinitely many times are polynomials of only finite number of other variables, $\left\{\tau_{k}^{\prime \Sigma}\right\}$, due to relations between $Z_{G}$ and $Z_{K}$ (see Table 2). We discuss this in Appendix I.

\subsection{Conjugation operators}

Let us explain now how one construct the conjugation operator in a manifest way. To this end, first of all one constructs the two-differentials

$$
f_{\hat{J}}\left(z, z^{\prime} \mid g^{2}\right)=\hat{J}\left(z \mid g^{2}\right) \hat{J}\left(z^{\prime} \mid g^{2}\right)-: \hat{J}\left(z \mid g^{2}\right) \hat{J}\left(z^{\prime} \mid g^{2}\right):=d_{z} h_{\hat{J}}\left(z, z^{\prime} \mid g^{2}\right)
$$

that can be made of both local and global currents. In particular,

$$
\begin{gathered}
f_{G}\left(z, z^{\prime} \mid g^{2}\right)=g^{2} \frac{d z d z^{\prime}}{2\left(z-z^{\prime}\right)^{2}}=g^{2} d_{z} \frac{d z}{2\left(z^{\prime}-z\right)} \\
f_{K}\left(z, z^{\prime} \mid g^{2}\right)=g^{2} \frac{\left(z^{2}+z^{\prime 2}\right) d z d z^{\prime}}{4\left(z^{2}-z^{\prime 2}\right)^{2}}=g^{2} d_{z} \frac{z d z^{\prime}}{4\left(z^{\prime 2}-z^{2}\right)} \\
f_{C}\left(z, z^{\prime} \mid g^{2}\right)=g^{2} \frac{z z^{\prime} d z d z^{\prime}}{2\left(z^{2}-z^{\prime 2}\right)^{2}}=g^{2} d_{z} \frac{z^{\prime} d z^{\prime}}{4\left(z^{\prime 2}-z^{2}\right)}
\end{gathered}
$$


Depending on its symmetry, the global current in the vicinity of a singular point $\xi$ is equivalent to one or another canonical local current listed in s.2.2,

$$
\hat{\mathbf{J}}\left(z \mid g^{2}\right) \sim \alpha_{\xi} \hat{J}^{\xi}\left(x \mid \beta_{\xi} g^{2}\right)
$$

for some constants $\alpha_{\xi}$ and $\beta_{\xi}$. This means that in the local coordinate in the vicinity of the point $\xi$, it is equal to

$$
\alpha\left(\hat{J}_{\xi}\left(x \mid \beta g^{2}\right)+\Delta_{\xi} \hat{J}^{\xi}\left(x \mid \beta_{\xi} g^{2}\right)\right)=\alpha_{\xi} e^{\hat{U}} \hat{J}^{\xi}\left(x \mid \beta_{\xi} g^{2}\right) e^{-\hat{U}}
$$

In its turn, it implies that

$$
f_{\hat{\mathbf{J}}}^{\xi \xi}\left(z(x), z^{\prime}\left(x^{\prime}\right) \mid g^{2}\right)-\alpha_{\xi}^{2} f_{\hat{\jmath} \xi}\left(x, x^{\prime} \mid \beta_{\xi} g^{2}\right)=A_{\hat{\mathbf{J}}}^{\xi \xi}\left(x, x^{\prime} \mid g^{2}\right)=\sum_{k, m=0}^{\infty} \alpha_{k m}^{\xi \xi} x^{k} x^{\prime m} d x d x^{\prime}
$$

Consider, e.g., the local currents at two infinities. They coincide with each other, and the conjugation operator is

$$
U_{\infty}=\frac{1}{2 \alpha_{\infty}^{2} g^{4} c_{\infty}^{2} \beta_{\infty}^{2}} \oint_{\infty} \oint_{\infty} A_{\hat{\mathbf{J}}}^{\infty}\left(x, x^{\prime} \mid g^{2}\right) \hat{\Omega}^{\infty}\left(x \mid \beta_{\infty} g^{2}\right) \hat{\Omega}^{\infty}\left(x^{\prime} \mid \beta_{\infty} g^{2}\right)+\oint_{\infty} k^{\infty}\left(z \mid \beta_{\infty} g^{2}\right) \hat{\Omega}^{\infty}\left(z \mid \beta_{\infty} g^{2}\right)
$$

where, depending on the local current associated with infinity (i.e. on the projection of the global current), $c_{\infty}$ is $\frac{1}{4}$ for the complex matrix model and the Kontsevich currents and $\frac{1}{2}$ for the Gaussian current $^{6}$. Emerging $k^{\infty}\left(s \mid \beta_{\infty} g^{2}\right)$ here is due to different shifts of global and local currents and it can be got from the difference of these currents ${ }^{7}$. Calculations for the branching points looks similar, the main difference being that there can be inequivalent points, as in examples s.5-8, and with help of the operator $\hat{U}$ one should compensate impacts of $f_{\hat{\mathbf{J}}}^{a b}$ with $a \neq b$. An accurate calculation shows that this changes the common sign, and one gets the result

$$
\hat{U}=\sum_{a, b} \frac{1}{2 \alpha_{a} \alpha_{b} g^{4} c_{a} c_{b} \beta_{a} \beta_{b}} \oint_{a} \oint_{b} A_{\hat{\mathbf{J}}}^{a b}\left(x, x^{\prime} \mid g^{2}\right) \hat{\Omega}^{a}\left(x \mid \beta_{a} g^{2}\right) \hat{\Omega}^{b}\left(x^{\prime} \mid \beta_{b} g^{2}\right)+\sum_{a} \oint_{a} k^{a}\left(z \mid \beta_{a} g^{2}\right) \hat{\Omega}^{a}\left(z \mid \beta_{a} g^{2}\right)
$$

$$
\begin{aligned}
& { }^{6} \text { While } \Delta \hat{J}^{\infty}\left(s \mid \beta_{\infty} g^{2}\right) \text { is manifestly } \\
& \begin{array}{l}
\Delta \hat{J}^{\infty}\left(s \mid \beta_{\infty} g^{2}\right)=-\left[\hat{J}^{\infty}\left(s \mid \beta g^{2}\right), \hat{U}_{\infty}\right]=\frac{1}{\alpha_{\infty}^{2} g^{4} c_{\infty}^{2} \beta^{2}} \oint_{\infty} \oint_{\infty} A_{\mathbf{J}}^{a a}\left(x, x^{\prime} \mid g^{2}\right) \hat{\Omega}^{\infty}\left(x \mid \beta_{\infty} g^{2}\right)\left[\hat{J}^{\infty}\left(s \mid \beta_{\infty} g^{2}\right), \hat{\Omega}^{\infty}\left(x^{\prime} \mid \beta_{\infty} g^{2}\right)\right]+ \\
+\oint_{\infty} k^{\infty}\left(x \mid \beta_{\infty} g^{2}\right)\left[\hat{J}^{\infty}\left(s \mid \beta_{\infty} g^{2}\right), \hat{\Omega}^{\infty}\left(x \mid \beta_{\infty} g^{2}\right)\right]=\frac{1}{\alpha_{\infty}^{2} g^{4} c_{\infty}^{2} \beta_{\infty}^{2}} \oint_{\infty} \oint_{\infty} A_{\hat{\mathbf{J}}}^{a a}\left(x, x^{\prime} \mid \beta_{\infty} g^{2}\right) \hat{\Omega}^{\infty}\left(x \mid \beta_{\infty} g^{2}\right) h_{\infty}\left(x^{\prime}, s \mid \beta_{\infty} g^{2}\right)+ \\
\quad+\oint_{\infty} k^{\infty}\left(x \mid \beta_{\infty} g^{2}\right) h_{\infty}\left(x, s \mid \beta_{\infty} g^{2}\right)=g^{2} c_{\infty} \beta_{\infty}\left(\frac{1}{\alpha_{\infty}^{2} g^{4} c_{\infty}^{2} \beta_{\infty}^{2}} \oint_{\infty} A_{\hat{\mathbf{J}}}^{a a}\left(x, s \mid g^{2}\right) \hat{\Omega}^{\infty}\left(x \mid \beta_{\infty} g^{2}\right)+k^{\infty}\left(s \mid \beta_{\infty} g^{2}\right)\right)
\end{array}
\end{aligned}
$$

${ }^{7}$ I.e. from

$$
\begin{gathered}
\left(\hat{J}^{\infty}\left(x \mid \beta_{\infty} g^{2}\right)+\Delta \hat{J}^{\infty}\left(x \mid \beta_{\infty} g^{2}\right)\right)\left(\hat{J}^{\infty}\left(x^{\prime} \mid \beta_{\infty} g^{2}\right)+\Delta \hat{J}^{\infty}\left(x^{\prime} \mid \beta_{\infty} g^{2}\right)\right)- \\
-:\left(\hat{J}^{\infty}\left(x \mid \beta_{\infty} g^{2}\right)+\Delta \hat{J}^{\infty}\left(x \mid \beta_{\infty} g^{2}\right)\right)\left(\hat{J}^{\infty}\left(x^{\prime} \mid \beta_{\infty} g^{2}\right)+\Delta \hat{J}^{\infty}\left(x^{\prime} \mid \beta_{\infty} g^{2}\right)\right):= \\
=f_{\hat{J} \infty}\left(x, x^{\prime} \mid \beta_{\infty} g^{2}\right)+\hat{J}^{\infty}\left(x \mid \beta_{\infty} g^{2}\right) \Delta J^{\infty}\left(x^{\prime} \mid \beta_{\infty} g^{2}\right)-: \hat{J}^{\infty}\left(x \mid \beta_{\infty} g^{2}\right) \Delta J^{\infty}\left(x^{\prime} \mid \beta_{\infty} g^{2}\right):+ \\
+\Delta J^{\infty}\left(x \mid \beta_{\infty} g^{2}\right) \hat{J}^{\infty}\left(x^{\prime} \mid \beta_{\infty} g^{2}\right)-: \Delta J^{\infty}\left(x \mid \beta_{\infty} g^{2}\right) \hat{J}^{\infty}\left(x^{\prime} \mid \beta_{\infty} g^{2}\right): \\
=\frac{1}{\alpha_{\infty}^{2} g^{2} c_{\infty} \beta_{\infty}} \oint_{\infty} A_{\hat{\mathbf{J}}}^{a a}\left(x^{\prime}, s \mid g^{2}\right)\left(\hat{J}^{\infty}\left(x \mid \beta_{\infty} g^{2}\right) \hat{\Omega}^{\infty}\left(s \mid \beta_{\infty} g^{2}\right)-: \hat{J}^{\infty}\left(x \mid \beta_{\infty} g^{2}\right) \hat{\Omega}^{\infty}\left(s \mid \beta_{\infty} g^{2}\right):\right)=\frac{1}{\alpha_{\infty}^{2}} A_{\hat{J}}^{a a}\left(x, x^{\prime} \mid g^{2}\right) \\
\Delta \hat{J}^{\infty}\left(x \mid \beta_{\infty} g^{2}\right) \hat{J}^{\infty}\left(x^{\prime} \mid \beta_{\infty} g^{2}\right)-: \Delta J^{\infty}\left(x \mid \beta_{\infty} g^{2}\right) \hat{J}^{\infty}\left(x^{\prime} \mid \beta_{\infty} g^{2}\right):=0
\end{gathered}
$$


where the sum runs over (inequivalent) branching points. In particular, in examples below these are two branching points. One finally gets as a result

$$
Z_{\infty}\left(t^{\infty} \mid \beta_{\infty} g^{2}\right)=e^{U_{\infty}} e^{\hat{U}} \prod_{a} Z_{a}\left(t^{a} \mid \beta_{a} g^{2}\right)
$$

\subsection{Summary: Relations between special functions}

Given a couple of projections of the same $\mathbf{Z}$ (say, $Z(T, S)$ or $Z(R, Q)$ ) from Table 1 , one can exclude the global partition function and obtain an additional relation between the other two special functions. In this way, any two special functions $Z_{G}, Z_{K}$ and $Z_{C}$ (and $Z_{A C K M}$ ) can be related.

Relations between these special functions and the global partition functions are summarized in the following tables ${ }^{8}$, where we write down only necessary part of interrelations, while all the remaining relations can be read off from these. Note that these tables are not symmetric, sometimes one trivially inverse the relation (say, between $Z_{G}(t)$ and $Z_{G}(\tilde{t})$ ), while sometimes it is impossible (like the relation between $Z_{G}$ and $Z_{K}$ ). In the former case we put in the corresponding cell of the table "S", in the latter case, we put the cross "X".

Table 2. Relations between local partition functions emerging from $\mathbf{Z}\left(T^{ \pm}\right)$

\begin{tabular}{|c|c|c|c|c|c|c|}
\hline & $\begin{array}{l}\mathbf{Z}\left(T^{ \pm}\right) \\
\text {eq. (2.9) }\end{array}$ & $\begin{array}{c}Z_{G}(t) \\
\text { eq. (2.10) }\end{array}$ & $\begin{array}{c}Z_{K}(\tau) \\
\text { eq. (2.13) }\end{array}$ & $\begin{array}{c}Z_{G}(\tilde{t}) \\
\text { eq.(2.23) }\end{array}$ & $\begin{array}{c}Z_{K}(\tilde{\tau}) \\
\text { eq. (2.19) }\end{array}$ & $\begin{array}{c}Z_{A C K M}\left(t^{ \pm}\right) \\
\text {eq. (2.28) }\end{array}$ \\
\hline $\mathbf{Z}\left(T^{ \pm}\right)$ & & $\begin{aligned} &=e^{-U_{G}} Z_{G}(t) \\
& t_{k}: 15.3 \\
& U_{G}: 5.10\end{aligned}$ & $\begin{array}{c}=e^{\hat{U}_{K}} Z_{K}\left(\tau_{+}\right) Z_{K}\left(\tau_{-}\right) \\
\tau_{k}: \text { (5.12), (6.1), (6.2) } \\
\hat{U}_{K}: \text { (5.18), 6.14 }\end{array}$ & & & \\
\hline$Z_{G}(t)$ & $S$ & & $\begin{array}{c}=e^{\hat{U}_{G K}} Z_{K}\left(\tau_{+}\right) Z_{K}\left(\tau_{-}\right) \\
\tau_{k}^{ \pm}:[6.2,6.31 \\
\hat{U}_{G K}=U_{G}+\hat{U}_{K}\end{array}$ & $\begin{array}{c}=e^{U_{G G}} Z_{G}(\tilde{t}) \\
t_{k}: 4.3 \\
U_{G G}: 4.7\end{array}$ & & \\
\hline$Z_{K}(\tau)$ & $\mathrm{X}$ & $\mathrm{X}$ & & $\mathrm{X}$ & $\begin{array}{c}=e^{\hat{U}_{K K}} Z_{K}(\tilde{\tau}) \\
\tilde{\tau}_{k}: 3.3 \\
\hat{U}_{K K}: 3.3\end{array}$ & $\mathrm{X}$ \\
\hline$Z_{G}(\tilde{t})$ & S & S & & & & \\
\hline$Z_{K}(\tilde{\tau})$ & $\mathrm{X}$ & $\mathrm{X}$ & S & $\mathrm{X}$ & & \\
\hline$Z_{A C K M}\left(t^{ \pm}\right)$ & $S$ & S & $\begin{array}{c}=: e^{\hat{U}_{G K}} Z_{K}\left(\tau_{+}\right) Z_{K}\left(\tau_{-}\right): \text {(7.17) } \\
t_{k}^{ \pm}: \text {17.15) } \\
\hat{U}_{G K}: \text { (7.6.17), (7.17) }\end{array}$ & S & & \\
\hline
\end{tabular}

Table 2 deals with projections of the global partition function $\mathbf{Z}\left(T^{ \pm}\right)$and relations between the four special functions which these projections generate. There are also similar relations for other projections of the global partition function. For example, the projection $\mathbf{Z}(T)$ gives rise to relations

\footnotetext{
${ }^{8}$ We do not need more than two global partition functions, say, $\mathbf{Z}(T, S)$ and $\mathbf{Z}(T)$ in order to give rise to all interrelations between local partition functions.
} 
between the complex matrix model and the Kontsevich partition function. We consider it for the case of the sphere with two additional singular points.

Table 3 deals with projections of the global partition function $\mathbf{Z}(T)$ and special functions $Z_{K}$ and $Z_{C}$.

Table 3. Relations between local partition functions emerging from $\mathbf{Z}(T)$

\begin{tabular}{|c|c|c|c|}
\hline & $\mathbf{Z}(T)$ & $Z_{C}(\mathfrak{t})$ & $Z_{K}(\tau) \tilde{Z}_{K}(\mathcal{T})$ \\
& & eq.(2.17) & \\
\hline \multirow{3}{*}{$\mathbf{Z}(T)$} & & $=e^{-V_{C}} Z_{C}(\mathfrak{t})$ & $=e^{V_{C}+\hat{V}_{\text {ram }}} Z_{K}(\tau) \tilde{Z}_{K}(\mathcal{T})$ \\
& & $\mathfrak{t}_{k}:(8.5)$ & $\tau_{k}:$ (8.10) $; \mathcal{T}_{k}:(8.13)$ \\
& & $V_{C}:(8.21)$ & $\hat{V}_{\text {ram }}:$ (88.22), (8.23) \\
\hline
\end{tabular}

These projections of $\mathbf{Z}(T)$ give rise to yet another decomposition formula, $Z_{C}(\mathfrak{t})=e^{\hat{V}_{C K}} Z_{K}(\tau) \tilde{Z}_{K}(\mathcal{T})$ with $\hat{V}_{C K}=V_{C}+\hat{V}_{K}$.

Remaining sections will be devoted to detailed description of particular cells in this table. They will be ordered so that simpler calculations precede the more sophisticated ones. The simplest are the relations involving vicinity of a single puncture and well known functions, i.e. between $Z_{G}(t)$ and $Z_{G}(\tilde{t})$ and between $Z_{K}(\tau)$ and $Z_{K}(\tilde{\tau})$. The next level of complexity involves the more sophisticated $\mathbf{Z}(T, S)$, but relations are still in vicinities of a single point: i.e. between $\mathbf{Z}(T, S)$ and other functions. After that, $\mathbf{Z}(T, S)$ can be eliminated to provide relations involving vicinities of different punctures. The same procedure is then repeated with the global function $\mathbf{Z}(T)$ leading to relations involving $Z_{C}(\mathfrak{t}), Z_{K}(\tau)$ and $\tilde{Z}_{K}(\mathcal{T})$. Further generalization to arbitrary (multi-cut, $n>1$ ) hyperelliptic curves and to Dijkgraaf-Vafa branches of Hermitean-model partition function, as well as to generic branches with genus expansion, 1] described in terms of the check-operators [13] will be considered elsewhere. 


\section{$3 \quad Z_{K}(\tau) \rightarrow Z_{K}(\tilde{\tau})$}

\subsection{Summary}

We start with the current

$$
\hat{J}_{K}(\xi \mid \tau)=\sum_{m=0}^{\infty}\left(\frac{2 m+1}{4} \tau_{m} \xi^{2 m} d \xi+g^{2} \frac{d \xi}{\xi^{2 m+2}} \frac{\partial}{\partial \tau_{m}}\right)
$$

A change of coordinate

$$
\tilde{\xi}=G^{1 / 3} \sqrt{\xi^{2}-u} \quad \text { or } \quad \xi=\sqrt{G^{-2 / 3} \tilde{\xi}^{2}+u},
$$

supplemented by transform of time-variables

$$
\tilde{\tau}_{m}=G^{-\frac{2 m+1}{3}} \sum_{k=0}^{\infty} \frac{\Gamma(m+k+3 / 2) u^{k}}{\Gamma(m+3 / 2) k !} \tau_{m+k},
$$

converts this current into itself,

$$
\hat{J}_{K}(\tilde{\xi} \mid \tilde{\tau})=\sum_{m=0}^{\infty}\left(\frac{2 m+1}{4} \tilde{\tau}_{m} \tilde{\xi}^{2 m} d \tilde{\xi}+g^{2} \frac{d \tilde{\xi}}{\tilde{\xi}^{2 m+2}} \frac{\partial}{\partial \tilde{\tau}_{m}}\right)
$$

plus an additive correction ${ }^{9}$

$$
\begin{gathered}
\hat{J}_{K}(\xi \mid \tau)=\hat{J}_{K}(\tilde{\xi} \mid \tilde{\tau})+\sum_{m<0} \frac{2 m+1}{4} \tilde{\tau}_{m} \tilde{\xi}^{2 m} d \tilde{\xi}+ \\
+g^{2}(\hat{\nabla}(\xi) u(\tau))\left(\hat{\tilde{\mathcal{L}}}_{-1}(\tilde{\tau})-\frac{1}{16 g^{2}}\right)-\frac{2 g^{2}}{3}(\hat{\nabla}(\xi) \log G(\tau))\left(\hat{\tilde{\mathcal{L}}}_{0}(\tilde{\tau})-\frac{1}{16}\right)
\end{gathered}
$$

which can be partly eliminated by twisting,

$$
\left(G^{1 / 12} e^{U_{K K}}\right) \hat{J}_{K}(\xi \mid \tau)\left(G^{-1 / 12} e^{-U_{K K}}\right)=\hat{J}_{K}(\tilde{\xi} \mid \tilde{\tau})+g^{2}(\hat{\nabla} u) \hat{\tilde{\mathcal{L}}}_{-1}+g^{2}(\hat{\nabla} \log G) \hat{\tilde{\mathcal{L}}}_{0}
$$

where function

$$
\begin{gathered}
U_{K K}=\frac{1}{2 g^{2}} \sum_{k, l=0}^{\infty} A_{k l}(u, G) \tau_{k} \tau_{l} \\
\sum_{k, l=0}^{\infty} A_{k l}(u, G) \tau_{l} \frac{d \xi}{\xi^{2 k+2}}=\frac{1}{2} \sum_{m=0}^{\infty}(m+1 / 2) \frac{d \tilde{\xi}}{\tilde{\xi}^{2 m+2}} \tilde{\tau}_{-m-1} \stackrel{3.3}{=} \\
=\frac{1}{2} G^{\frac{2 m+1}{3}} \sum_{k, m=0}^{\infty}\left(m+\frac{1}{2}\right) \frac{\Gamma\left(k-m+\frac{1}{2}\right)}{\Gamma\left(\frac{1}{2}-m\right)} \frac{u^{k}}{k !} \frac{d \tilde{\xi}}{\tilde{\xi}^{2 m+2}} \tau_{k-m-1} \\
A_{k l}=\frac{\Gamma(k+3 / 2) \Gamma(l+3 / 2) u^{k+l+1}}{2 \Gamma(1 / 2)^{2} k ! l !(k+l+1)}=A_{l k}
\end{gathered}
$$

Since $Z_{K}(\tilde{\tau})$ is annihilated by Virasoro constraints, including $\hat{\tilde{L}}_{-1}^{K}$ and $\hat{\tilde{L}}_{0}^{K}$, it follows (see comments below) that

$$
Z_{K}(\tau)=G^{-1 / 24} e^{U_{K K}(\tau)} Z_{K}(\tilde{\tau})
$$

\footnotetext{
${ }^{9} \hat{\nabla}(\xi)$ is defined in (2.10).
} 
for arbitrary choice of $u$ and $G$. Since (3.9) is an identity in $u$ and $G$, these parameters are allowed to depend on $\tau$ in an arbitrary way. This freedom can be used to impose two arbitrary constraints on $\tilde{\tau}$. In particular, one can define moment variables as follows (see s.2.5.2):

$$
\begin{gathered}
\text { put } \tilde{\tau}_{0}=\tilde{\tau}_{1}^{\Sigma}=0, \\
\text { then } U_{K K}=\frac{1}{g^{2}} \mathcal{F}^{(0)}(\tau), \quad-\frac{1}{24} \log G=\mathcal{F}^{(1)}(\tau),
\end{gathered}
$$

while $\mathcal{F}^{(0)}(\tilde{\tau})=\mathcal{F}^{(1)}(\tilde{\tau})=0$, and $\mathcal{F}^{(p)}(\tilde{\tau})$ with $p \geq 2$ are polynomials

of a finite ( $p$-dependent) order in finite ( $p$-dependent) number of a few first $\tilde{\tau}-$ variables.

\subsubsection{Comments on the derivation}

Triangular relation (3.3)

$$
\tilde{\tau}_{m}=G^{-\frac{2 m+1}{3}} \sum_{k=0}^{\infty} \frac{\Gamma(m+k+3 / 2) u^{k}}{\Gamma(m+3 / 2) k !} \tau_{m+k}
$$

follows from equality of terms $\xi^{2 m} d \xi$ with $m \geq 0$ in $\hat{J}_{K}(\xi)$ and $\hat{J}_{K}(\tilde{\xi})$, which are singular at infinity $\xi \sim \tilde{\xi} \sim \infty$.

In terms, which vanish at infinity, it is convenient first to consider the case of $\tau$-independent $u$ and G. Then

$$
\hat{\nabla}(\xi) \equiv \sum_{m \geq 0}^{\infty} \frac{d \xi}{\xi^{2 m+2}} \frac{\partial}{\partial \tau_{m}}=\sum_{m \geq 0}^{\infty} \frac{d \tilde{\xi}}{\tilde{\xi}^{2 m+2}} \frac{\partial}{\partial \tilde{\tau}_{m}} \equiv \hat{\tilde{\nabla}}(\tilde{\xi})
$$

and the discrepancy between the two currents,

$$
\hat{J}_{K}(\xi \mid \tau)-\hat{J}_{K}(\tilde{\xi} \mid \tilde{\tau})=\frac{1}{4} \sum_{m<0}(2 m+1) \tilde{\tau}_{m} \tilde{\xi}^{2 m} d \tilde{\xi}=-\frac{1}{4} \sum_{m=0}^{\infty}(2 m+1) \tilde{\tau}_{-m-1} \frac{d \tilde{\xi}}{\tilde{\xi}^{2 m+2}}
$$

is eliminated by twisting with $U_{K K}$.

Relation (3.12) is a corollary of (3.11) for constant $u$ and $G$

$$
\frac{\partial}{\partial \tau_{n}}=\sum_{m=0}^{\infty} \frac{\partial \tilde{\tau}_{m}}{\partial \tau_{n}} \frac{\partial}{\partial \tilde{\tau}_{m}}=\sum_{m=0}^{n} G^{-\frac{2 m+1}{3}} \frac{\Gamma(n+3 / 2) u^{n-m}}{\Gamma(m+3 / 2)(n-m) !} \frac{\partial}{\partial \tilde{\tau}_{m}}
$$

Inverting this triangular relation, we obtain

$$
\frac{\partial}{\partial \tilde{\tau}_{m}}=G^{\frac{2 m+1}{3}} \sum_{0 \leq k \leq m} \frac{\Gamma(m+3 / 2)}{\Gamma(m-k+3 / 2)} \frac{(-)^{k} u^{k}}{k !} \frac{\partial}{\partial \tau_{m-k}}
$$

If $u$ and $G$ are $\tau$-dependent, additional terms are added to the r.h.s. of (3.14):

$$
\begin{gathered}
\frac{\partial u}{\partial \tau_{n}} \sum_{m=0}^{\infty} \frac{\partial \tilde{\tau}_{m}}{\partial u} \frac{\partial}{\partial \tilde{\tau}_{m}}+\frac{\partial \log G}{\partial \tau_{n}} \sum_{m=0}^{\infty} \frac{\partial \tilde{\tau}_{m}}{\partial \log G} \frac{\partial}{\partial \tilde{\tau}_{m}}= \\
=\frac{\partial u}{\partial \tau_{n}}\left(\sum_{m=0}^{\infty}(m+3 / 2) \tilde{\tau}_{m+1} \frac{\partial}{\partial \tilde{\tau}_{m}}\right)+\frac{\partial \log G}{\partial \tau_{n}}\left(-\frac{2}{3} \sum_{m=0}^{\infty}(m+1 / 2) \tilde{\tau}_{m} \frac{\partial}{\partial \tilde{\tau}_{m}}\right)= \\
=\frac{\partial u}{\partial \tau_{n}}\left(\tilde{\mathcal{L}}_{-1}-\frac{1}{16} \tilde{\tau}_{0}^{2}\right)-\frac{2}{3} \frac{\partial \log G}{\partial \tau_{n}}\left(\tilde{\mathcal{L}}_{0}-\frac{1}{16}\right),
\end{gathered}
$$

so that instead of (3.12) we have:

$$
\hat{\nabla}(\xi)=\hat{\tilde{\nabla}}(\tilde{\xi})+(\hat{\nabla}(\xi) u)\left(\hat{\tilde{\mathcal{L}}}_{-1}(\tilde{\tau})-\frac{\tilde{\tau}_{0}^{2}}{16 g^{2}}\right)-\frac{2}{3}(\hat{\nabla}(\xi) \log G)\left(\hat{\tilde{\mathcal{L}}}_{0}(\tilde{\tau})-\frac{1}{16}\right)
$$


When acting on $Z_{K}(\tilde{\tau})$, which is annihilated by $\hat{\tilde{\mathcal{L}}}_{n}$ with $n \geq-1$, including $n=-1$ and $n=0$, additional terms produce

$$
-\frac{\tilde{\tau}_{0}^{2}}{16 g^{2}}(\hat{\nabla}(\xi) u)+\frac{1}{24}(\hat{\nabla}(\xi) \log G)
$$

which coincides with additional terms

$$
(\hat{\nabla}(\xi) u) \frac{\partial U_{K K}}{\partial u}+(\hat{\nabla}(\xi) \log G)\left(\frac{\partial U_{K K}}{\partial \log G}+\frac{1}{24}\right)
$$

coming from twisting the l.h.s. of (3.17) with $G^{1 / 24} e^{-U_{K K}}$. Here we used that

$$
\begin{gathered}
\frac{\partial U_{K K}}{\partial \log G}=0 \\
\frac{\partial U_{K K}}{\partial u}=\frac{\tilde{\tau}_{0}^{2}}{16 g^{2}}
\end{gathered}
$$

Taking a square of (3.6) we have: For $\hat{J}_{K}=d \hat{\varphi}$

$$
\begin{gathered}
\hat{\tilde{J}}_{K}{ }^{2}(\tilde{\xi})=\tilde{T}(\tilde{\xi})+d\left(\left(\hat{\tilde{\nabla}}_{\tilde{\xi}} \tilde{\xi}\right) \hat{\tilde{J}}_{K}(\tilde{\xi})\right) \\
{\left[\hat{\tilde{\mathcal{L}}}_{-1}, \hat{\tilde{J}}_{K}(\tilde{\xi})\right]=d\left(\hat{\tilde{J}}_{K}(\tilde{\xi})\left(\frac{1}{\tilde{\xi}}+\frac{1}{\tilde{\xi}}\left[\hat{\tilde{\mathcal{L}}}_{-1}, u\right]+\tilde{\xi}\left[\hat{\tilde{\mathcal{L}}}_{-1}, \log G\right]\right)\right)} \\
{\left[\hat{\tilde{\mathcal{L}}}_{0}, \hat{\tilde{J}}_{K}(\tilde{\xi})\right]=d\left(\hat{\tilde{J}}_{K}(\tilde{\xi})\left(\tilde{\xi}+\frac{1}{\tilde{\xi}}\left[\hat{\tilde{\mathcal{L}}}_{0}, u\right]+\tilde{\xi}\left[\hat{\tilde{\mathcal{L}}}_{0}, \log G\right]\right)\right)}
\end{gathered}
$$

so that

$$
\begin{gathered}
\left(\hat{J_{K}}(\tilde{\xi} \mid \tilde{\tau})+g^{2}(\hat{\nabla} u) \hat{\tilde{\mathcal{L}}}_{-1}+g^{2}(\hat{\nabla} \log G) \hat{\tilde{\mathcal{L}}}_{0}\right) \hat{J}_{K}(\tilde{\xi} \mid \tilde{\tau}) Z_{K}(\tilde{\tau})= \\
=\left(\hat{\tilde{T}}(\tilde{\xi})+d\left\{\hat { \tilde { J } } _ { K } ( \tilde { \xi } ) \left[\hat{\nabla}-\hat{\tilde{\nabla}}+g^{2}(\hat{\nabla} u)\left(\hat{\tilde{\mathcal{L}}}_{-1}-\frac{1}{16 g^{2}}\right)-\right.\right.\right. \\
\left.\left.\left.-\frac{2 g^{2}}{3}(\hat{\nabla} \log G)\left(\hat{\tilde{\mathcal{L}}}_{0}-\frac{1}{16}\right), \frac{1}{\tilde{\xi}} u+\tilde{\xi} \log G\right]\right\}\right) Z_{K}(\tilde{\tau})=\hat{\tilde{T}}(\tilde{\xi}) Z_{K}(\tilde{\tau})
\end{gathered}
$$

since operator in the commutator vanishes due to (3.17). Note that $c$-numbers $1 / 16 g^{2}$ and $1 / 16$ do not affect the value of the commutator, but are needed to make the operator vanishing.

One more (almost identical) way to check consistency is to check that the r.h.s. of (3.9) is indeed independent of $u$ and $G$ :

$$
\begin{gathered}
\frac{1}{2 g^{2}}\left(\sum_{k, l=0}^{\infty} \frac{\partial A_{k l}}{\partial u} \tau_{k} \tau_{l}\right) Z_{K}(\tilde{\tau})+\sum_{m=0}^{\infty} \frac{\partial \tilde{\tau}_{m}}{\partial u} \frac{\partial Z_{K}(\tilde{\tau})}{\partial \tau_{m}}= \\
=\frac{1}{2 g^{2}}\left(\sum_{k, l=0}^{\infty} \frac{\partial A_{k l}}{\partial u} \tau_{k} \tau_{l}\right) Z_{K}(\tilde{\tau})+\left(\tilde{\mathcal{L}}_{-1}-\frac{1}{16 g^{2}} \tilde{\tau}_{0}^{2}\right) Z_{K}(\tilde{\tau})=0
\end{gathered}
$$

because of (3.21). and

$$
\begin{aligned}
& -\frac{1}{24} Z_{K}(\tilde{\tau})+\frac{1}{2}\left(\sum_{k, l=0}^{\infty} \frac{\partial A_{k l}}{\partial \log G} \tau_{k} \tau_{l}\right) Z_{K}(\tilde{\tau})+\sum_{m=0}^{\infty} \frac{\partial \tilde{\tau}_{m}}{\partial \log G} \frac{\partial Z_{K}(\tilde{\tau})}{\partial \tau_{m}}= \\
= & -\frac{1}{24} Z_{K}(\tilde{\tau})+\frac{1}{2}\left(\sum_{k, l=0}^{\infty} \frac{\partial A_{k l}}{\partial \log G} \tau_{k} \tau_{l}\right) Z_{K}(\tilde{\tau})-\frac{2}{3}\left(\tilde{\mathcal{L}}_{0}-\frac{1}{16}\right) Z_{K}(\tilde{\tau})=0
\end{aligned}
$$

because of (3.20). 


\subsubsection{Comments on generic change of variables}

Make now arbitrary change of variables in the vicinity of $\xi=\infty$, consistent with the $\hat{J}_{K}(-\xi)=-\hat{J}_{K}(\xi)$ of the current:

$$
\xi \rightarrow \tilde{\xi}(\xi)=G^{1 / 3} \xi\left(1+\frac{b_{1}}{G^{2 / 3} \xi^{2}}+\ldots+\frac{b_{k}}{G^{2 k / 3} \xi^{2 k}}+\ldots\right)
$$

The coefficients $b_{k}$ can be either constants or functions of time-variables $\tau$. We omit $G$ below.

Introduce non-linear combinations $C_{m, k}$ and $B_{m, k}$ of the coefficients $b_{k}$ :

$$
\tilde{\xi}^{2 m+1}=\sum_{k=0}^{\infty} B_{m, k} \xi^{2 m+1-2 k}
$$

and

$$
\xi^{2 m+1}=\sum_{k=0}^{\infty} C_{m, k} \tilde{\xi}^{2 m+1-2 k}
$$

Then, from

$$
\sum_{m=0}^{\infty} \tau_{m} d \xi^{2 m+1}=\sum_{m=0}^{\infty} \tilde{\tau}_{m} d \tilde{\xi}^{2 m+1}
$$

we read

$$
\tilde{\tau}_{m}=\sum_{k \geq 0} C_{m+k, k} \tau_{m+k}
$$

and

$$
\frac{\partial}{\partial \tau_{m}}=\sum_{0 \leq k \leq m} C_{m, m-k} \frac{\partial}{\partial \tilde{\tau}_{k}}+O\left(\frac{\partial C}{\partial \tau}\right)
$$

For $\tau$-independent coefficients $B$ (and thus $C$ ) one obtains

$$
\sum_{m=0}^{\infty} \frac{d \xi}{\xi^{2 m+2}} \frac{\partial}{\partial \tau_{m}}=\sum_{m=0}^{\infty} \frac{d \tilde{\xi}}{\tilde{\xi}^{2 m+2}} \frac{\partial}{\partial \tilde{\tau}_{m}}
$$

since

$$
\sum_{m \geq k}^{\infty} \frac{C_{m, m-k} \xi^{-2 m-1}}{2 m+1}=\frac{\tilde{\xi}^{-2 k-1}}{2 k+1}
$$

or

$$
(m+1 / 2) C_{m+k, k}=(m+k+1 / 2) B_{-m-1, k}
$$

For the square-root change of the local parameter (3.2) with $G=1$

$$
C_{m, k}=\frac{\Gamma(m+3 / 2)}{\Gamma(m-k+3 / 2)} \frac{u^{k}}{k !}, \quad B_{m, k}(u)=C_{m, k}(-u)
$$

and one can easily check (3.36) is fulfilled. More generally, put

$$
\tilde{\xi}=\xi\left(1+\frac{a}{\xi^{2}}+\frac{b}{\xi^{4}}+\ldots\right), \quad \text { i.e. } \quad \xi=\tilde{\xi}\left(1-\frac{a}{\tilde{\xi}^{2}}-\frac{a^{2}+b}{\tilde{\xi}^{4}}-\ldots\right)
$$

Then

$$
\begin{gathered}
C_{m, 0}=B_{m, 0}=1 ; \quad C_{m, 1}=-(2 m+1) a, \quad B_{-m-1,1}=-(2 m+1) a \\
C_{m, 2}=(2 m+1)\left((m-1) a^{2}-b\right), \quad B_{-m-1,2}=(2 m+1)\left((m+1) a^{2}-b\right) ; \quad \text { etc }
\end{gathered}
$$

One can also easily check these coefficients satisfy (3.36). 
Using definition (3.7), one can construct the matrix $A_{k l}$ for this generic change of variables. The result is

$$
A_{k l}=\frac{1}{2} \sum_{n=0}^{l}\left(l+\frac{1}{2}\right) C_{k, n+k+1} B_{-n-1, l-n}
$$

Using (3.36), it can be rewritten as

$$
A_{k l}=\frac{1}{2} \sum_{n=0}^{l}\left(n+\frac{1}{2}\right) C_{l, l-n} C_{k, n+k+1}
$$

This matrix $A_{k l}$ is to be symmetric for the conjugation operator to exist. Let us check this is really the case ${ }^{10}$. To this end, one can convolute $A_{k l}$ in the form (3.40) with $\frac{x^{-2 k-1}}{(2 k+1)} \frac{y^{-2 l-1}}{(2 l+1)}$ to produce the generating function $A(x, y)$. Then, one can use formula (3.35) both for positive and negative $k$ to obtain

$$
A(x, y) \equiv \sum_{k, l=0}^{\infty} A_{k l} \frac{x^{-2 k-1}}{(2 k+1)} \frac{y^{-2 l-1}}{(2 l+1)}=\frac{1}{16}\left(\log \left[\frac{x+y}{x-y}\right]^{2}-\log \left[\frac{\tilde{x}(x)+\tilde{y}(y)}{\tilde{x}(x)-\tilde{y}(y)}\right]^{2}\right)
$$

where $\tilde{x}(x)$ and $\tilde{y}(y)$ are accordingly functions of $x$ and $y$ given by (3.28). It follows from this general formula that $A(x, y)$ is a symmetric function of $x$ and $y$ and, therefore, the matrix $A_{k l}$ is symmetric.

One can also "independently" derive formula (3.41) using the tools developed in s.2.6. Indeed, using formulas (2.50) and (2.44), one can easily understand that the second derivative of $A(x, y)$ w.r.t. $x$ and $y$ is equal to the difference of the two $f_{K}$ 's, up to the factor of $\frac{1}{g^{2}}$,

$$
\frac{1}{g^{2}} \frac{\partial^{2} A(x, y)}{\partial x \partial y}=f_{K}\left(x, y \mid g^{2}\right)-f_{K}\left(\tilde{x}(x), \tilde{y}(y) \mid g^{2}\right)
$$

These latter can be read off from the second formula of (2.41), and finally the result is

$$
\frac{\partial^{2} A(x, y)}{\partial x \partial y}=\frac{1}{4}\left[\frac{\left(x^{2}+y^{2}\right) d x d y}{\left(x^{2}-y^{2}\right)^{2}}-\frac{\left(\tilde{x}^{2}+\tilde{y}^{2}\right) d \tilde{x} d \tilde{y}}{\left(\tilde{x}^{2}-\tilde{y}^{2}\right)^{2}}\right]
$$

Upon integrating twice, this formula immediately leads to (3.41).

Thus, we have proved that, for the generic change of the local parameter, there exists a conjugation operator that intertwines between the currents $\hat{J}_{K}(\xi \mid \tau)$ and $\hat{J}_{K}(\tilde{\xi} \mid \tilde{\tau})$. However, this is not the case for the corresponding Virasoro algebras: the projector generally is not invariant under this change of the local parameter. If one requires the Virasoro algebras would map to each other, this restricts the generic changes of variables to the square-root one (3.2).

\footnotetext{
${ }^{10}$ It is easy to check with the explicit expressions (3.38) for several first entries that $A_{k l}$ is symmetric: $A_{0,1}=A_{1,0}$, $A_{2,0}=A_{0,2}$ etc
} 
$4 \quad Z_{G}(t) \rightarrow Z_{G}(\tilde{t})$

\subsection{Summary}

Now we start with the current

$$
\hat{J}_{G}(z \mid t)=\sum_{k=0} \frac{1}{2} k t_{k} z^{k-1} d z+\frac{S d z}{z}+g^{2} \sum_{k=1} \frac{d z}{z^{k+1}} \frac{\partial}{\partial t_{k}}
$$

A change of coordinate

$$
z=a \tilde{z}+b
$$

supplemented by transform of time-variables

$$
m \tilde{t}_{m}=\oint_{\infty} \frac{d v(z)}{\tilde{z}^{m}}=a \oint_{\infty} \frac{\sum_{k} k t_{k}(a z+b)^{n-1} d z}{z^{m}}=a^{m} \sum_{k} \frac{k ! b^{k-m}}{(m-1) !(k-m) !} t_{k}
$$

converts this current into itself,

$$
\hat{J}_{G}(\tilde{z} \mid \tilde{t})=\sum_{k=0} \frac{1}{2} k \tilde{t}_{k} \tilde{z}^{k-1} d \tilde{z}+\frac{S d \tilde{z}}{\tilde{z}}+g^{2} \sum_{k=1} \frac{d \tilde{z}}{\tilde{z}^{k+1}} \frac{\partial}{\partial \tilde{t}_{k}}
$$

plus an additive correction

$$
\hat{J}_{G}(z \mid t)=\hat{J}_{G}(\tilde{z} \mid \tilde{t})+S \frac{d \tilde{z}}{\tilde{z}} \sum_{k=0}^{\infty}\left(-\frac{b}{a \tilde{z}}\right)^{k}+g^{2}[\hat{\nabla}(z) \log a]\left[\hat{\tilde{L}}_{0}(\tilde{t})-S^{2}\right]+g^{2}[\hat{\nabla}(z) b] \hat{\tilde{L}}_{-1}(\tilde{t})
$$

which can be partly eliminated by twisting

$$
\left(a^{S^{2}} e^{U_{G G}}\right) \hat{J}_{G}(z \mid t)\left(a^{-S^{2}} e^{-U_{G G}}\right)=\hat{J}_{G}(\tilde{z} \mid \tilde{t})+g^{2}(\hat{\nabla} \log a) \tilde{\hat{L}}_{0}+g^{2}(\hat{\nabla} b) \tilde{\hat{L}}_{-1}
$$

where function

$$
U_{G G}=\frac{S}{g^{2}} \sum_{k=1}^{\infty} b^{k} t_{k}=\frac{S}{g^{2}} \sum_{k=1}^{\infty}\left(-\frac{b}{a}\right)^{k} \tilde{t}_{k}
$$

Since $Z_{G}(\tilde{t})$ is annihilated by the Virasoro constraints, including $\hat{\tilde{L}}_{-1}^{G}$ and $\hat{\tilde{L}}_{0}^{G}$, it follows (see comments below) that

$$
Z_{G}(t)=a^{S^{2}} e^{U_{G G}(t)} Z_{G}(\tilde{t})
$$

for arbitrary choice of $a$ and $b$. Since (4.8) is an identity in $a$ and $b$, these parameters are allowed to depend on $t$ in an arbitrary way. This freedom can be used to impose two arbitrary constraints on $\tilde{t}$.

\subsection{Comments}

Let us check that $Z_{G}$ in fact does not depend on $a$ and $b$ :

$$
Z_{G}^{-1} \partial_{b} Z_{G}=S \sum_{k=1} k b^{k-1} t_{k}+Z_{G}(\tilde{t})^{-1} \sum_{k=1} \frac{\partial \tilde{t}_{k}}{\partial b} \frac{\partial}{\partial \tilde{t}_{k}} Z_{G}(\tilde{t})=\frac{1}{a Z_{G}(\tilde{t})}\left(\sum_{k=1}(k+1) \tilde{t}_{k+1} \frac{\partial}{\partial \tilde{t}_{k}}+S \tilde{t}_{1}\right) Z_{G}\left(\tilde{t}_{t}\right)=0
$$

and

$$
\partial_{a} Z_{G}=\frac{S^{2}}{a}+\frac{1}{a} \sum_{k=1} k \tilde{t}_{k} \frac{\partial}{\partial \tilde{t}_{k}} Z_{G}(\tilde{t})=0
$$




\section{$5 \quad$ Projections of $\mathbf{Z}(T, S)$}

For the sake of simplicity, we consider here the symmetric model. The generic model is considered in s.7.

\section{1 $\mathrm{Z}(T, S)$ to $Z_{G}(t)$. Summary}

Projecting means one should establish equality, up to conjugation, of the global current and the local current $\hat{J}_{G}$ at the vicinity of infinity, i.e.

$$
\frac{1}{2} k t_{k} \cong \oint_{\infty} z^{-k} \hat{\mathbf{J}^{\mathbf{o}}}, \quad \frac{\partial}{\partial t_{k}} \cong \oint_{\infty} z^{k} \hat{\mathbf{J}^{\mathbf{o}}}
$$

where the sign $\cong$ means equality modulo possible conjugation, which does not affect commutation relations. In fact, the first equality is exact and gives rise to the change of time variables,

$$
\frac{1}{2} k t_{k}=\oint_{\infty} z^{-k} \hat{\mathbf{J}^{\mathbf{o}}}
$$

and, inversely (see notations in (2.10) $)$,

$$
\begin{aligned}
\left(k+\frac{1}{2}\right) T_{k} & =2 \oint_{\infty} \frac{z \hat{J}_{G}(z)}{y^{2 k+1}}, \quad\left(k+\frac{1}{2}\right) S_{k}=2 \oint_{\infty} \frac{\hat{J}_{G}(z)}{y^{2 k+1}} \\
\widetilde{T}_{k} & =\oint_{\infty} \frac{v(z) d z}{y^{2 k+3}}, \quad \widetilde{S}_{k}=\oint_{\infty} \frac{z v(z) d z}{y^{2 k+3}}
\end{aligned}
$$

In accordance with these formulas, $T_{0}$ contains $\frac{\partial}{\partial t_{0}}$. However, as we mentioned above, one may deal with $\frac{\partial}{\partial t_{0}}$ as with a $c$-number (equal to $\frac{S}{g^{2}}$ when acting on $Z_{G}$ ) and, moreover, to make the Legandre transform to replace it with $t_{0}^{\prime}$.

In order to reproduce the second equality in (5.1), one needs a non-trivial conjugation operator,

$$
\frac{\partial}{\partial t_{k}}=e^{U_{G}}\left(\oint_{\infty} z^{k} \hat{\mathbf{j} o}\right) e^{-U_{G}}
$$

We construct it following s.2.6, the result being the sum of two pieces, the first one is

$$
U_{G, 1}=\frac{1}{2} \oint_{\infty} \oint_{\infty} A_{\mathbf{J}}^{\infty \infty}\left(z, z^{\prime}\right) \hat{\Omega}_{G}(z) \hat{\Omega}_{G}\left(z^{\prime}\right) d z d z^{\prime}=\frac{1}{2} \oint_{\infty} \oint_{\infty} \rho_{G}^{(0 \mid 2)}\left(z_{1}, z_{2}\right) v^{\Sigma}\left(z_{1}\right) v^{\Sigma}\left(z_{2}\right) d z_{1} d z_{2}
$$

where

$$
A_{\mathbf{J}}^{\infty_{+} \infty_{+}}\left(z, z^{\prime}\right)=-A_{\mathbf{J}}^{\infty_{+} \infty_{-}}\left(z, z^{\prime}\right)=A_{\mathbf{J}}^{\infty_{-} \infty_{-}}\left(z, z^{\prime}\right)=\rho_{G}^{(0 \mid 2)}\left(z, z^{\prime}\right) d z d z^{\prime}
$$

and

$$
\rho_{G}^{(0 \mid 2)}\left(z_{1}, z_{2}\right)=\frac{d z_{1} d z_{2}}{2\left(z_{1}-z_{2}\right)^{2}}\left(\frac{z_{1} z_{2}-4 S}{y\left(z_{1}\right) y\left(z_{2}\right)}-1\right)
$$

is the two-point resolvent, [1, $v^{\Sigma}(z) \equiv \sum t_{k}^{\Sigma} z^{k}$, for other notations see (2.10).

The second piece, as explained in s.2.6, comes from noting that the shift for the global current is to be proportional to the 1 -form ${ }^{11} \Omega_{D V}=y(z) d z$ in order to reproduce the corresponding shift in $J_{G}$. It is

$$
\delta \hat{J}_{G}=-\frac{y(z) d z}{2}
$$

at the vicinity of infinity, can be also reproduced by the (still $c$-number) conjugation operator ${ }^{12}$ :

$$
U_{G, 2}=\oint_{\infty}\left(-\frac{y(z) d z}{2}+z d z\right) \hat{\Omega}^{\infty}(z)=\oint \rho_{G}^{(0 \mid 1)} v^{\Sigma}(z) d z
$$

\footnotetext{
${ }^{11}$ Considering $y^{n}(z) d z$ instead would lead to higher critical points of the same Gaussian branch.

${ }^{12}$ This is a priori clear from the fact that, unlike changing the polynomial part of the potential [1], adding any negative powers does not change the structure of solutions to the loop equations.
} 
where $\rho_{G}^{(0 \mid 1)}$ is the one-point resolvent, [1]. Thus, finally one has

$$
\begin{gathered}
\mathbf{Z}(T, S)=e^{-U_{G}} Z_{G}(t) \\
U_{G}=\frac{1}{g} \oint \rho^{(0 \mid 1)}(z) v^{\Sigma}(z)+\frac{1}{2 !} \oint \rho^{(0 \mid 2)}\left(z_{1}, z_{2}\right) v^{\Sigma}\left(z_{1}\right) v^{\Sigma}\left(z_{2}\right)
\end{gathered}
$$

\section{$5.2 \mathrm{Z}(T, S)$ to $Z_{K}(\tau)$. Summary}

In the vicinities of ramification points we not fix the local parameters, just put them to be a series

$$
z \pm a=\sum_{k=1}^{\infty} \alpha_{k}^{ \pm} x_{ \pm}^{2 k}
$$

with arbitrary coefficients $\alpha_{k}^{ \pm}$and non-zero $\alpha_{1}^{ \pm}$. Here we choose $\alpha_{k}^{+}=\alpha_{k}^{-}$, postponing the generic case until s.7. In the next section, we consider an explicit example of choice of the local parameter.

Projecting in the vicinity of ramification points provides us with the first example when, of two equalities

$$
(2 k+1) \tau_{k}^{ \pm} \cong \oint_{\infty} x_{ \pm}^{-(2 k+1)} \hat{\mathbf{J}^{\mathbf{o}}}, \quad \frac{\partial}{\partial \tau_{k}^{ \pm}} \cong \oint_{\infty} x_{ \pm}^{2 k+1} \hat{\mathbf{J}^{\mathbf{o}}}
$$

the second equality does not require conjugation,

$$
\frac{1}{2} \frac{\partial}{\partial \tau_{k}^{ \pm}}=\oint_{a_{ \pm}} x_{ \pm}^{2 k+1} \hat{\mathbf{J}^{\mathbf{o}}}
$$

while the first one does. This leads to conjugation operator which is an exponential of quadratic form (with constant coefficients) in time derivatives, not in times as in the examples above:

$$
\left(k+\frac{1}{2}\right) \tau_{k}^{ \pm}=e^{\hat{U}_{K}}\left(\oint_{\infty} x_{ \pm}^{-k} \hat{\mathbf{J}^{\mathbf{o}}}\right) e^{-\hat{U}_{K}}
$$

with

$$
\begin{gathered}
\hat{U}_{K}=\hat{U}_{K, 1}+U_{K, 2} \\
\hat{U}_{K, 1}=\frac{1}{2} \sum_{a, b=a_{ \pm}} \oint_{a} \oint_{b} A_{\mathbf{J}}^{a b}\left(z, z^{\prime}\right) \hat{\Omega}_{K}^{a}(z) \hat{\Omega}_{K}^{b}\left(z^{\prime}\right)= \\
=\frac{1}{4} \oint_{a_{+}} \oint_{a_{+}}\left(\frac{d z_{1} d z_{2}}{2\left(z_{1}-z_{2}\right)^{2}} \frac{z_{1} z_{2}-4 S}{y\left(z_{1}\right) y\left(z_{2}\right)}-\frac{d x_{1} d x_{2}\left(x_{1}^{2}+x_{2}^{2}\right)}{\left(x_{1}^{2}-x_{2}^{2}\right)^{2}}\right) \hat{\Omega}_{K}^{a_{+}}\left(x_{1}\right) \hat{\Omega}_{K}^{a_{+}}\left(x_{2}\right)+ \\
+\frac{1}{4} \oint_{a_{+}} \oint_{a_{-}}\left(\frac{d z_{1} d z_{2}}{2\left(z_{1}-z_{2}\right)^{2}} \frac{z_{1} z_{2}-4 S}{y\left(z_{1}\right) y\left(z_{2}\right)}\right) \hat{\Omega}_{K}^{a_{+}}\left(x_{1}\right) \hat{\Omega}_{K}^{a_{-}}\left(x_{2}\right)= \\
=\frac{1}{4} \sum_{a=a_{ \pm}} \oint_{a_{+}} \oint_{a}\left(\frac{d z_{1} d z_{2}}{2\left(z_{1}-z_{2}\right)^{2}} \frac{z_{1} z_{2}-4 S}{y\left(z_{1}\right) y\left(z_{2}\right)}\right) \hat{\Omega}_{K}^{a_{+}}\left(x_{1}\right) \hat{\Omega}_{K}^{a}\left(x_{2}\right)
\end{gathered}
$$

Again the shift

$$
\delta \hat{J}_{K}=-\frac{y(z) d z}{2}
$$

at the vicinities of the ramification points can be reproduced by the conjugation operator

$$
\hat{U}_{K, 2}=\sum_{a=a_{ \pm}} \oint_{a} \frac{1}{2}\left(y(z) d z-x(z)^{2} d x(z) \oint_{a} \frac{y\left(z^{\prime}\right) d z^{\prime}}{x\left(z^{\prime}\right)^{3}}\right) \hat{\Omega}_{K}^{a}(x(z))
$$

Thus, finally one has

$$
\begin{gathered}
\mathbf{Z}(T, S)=e^{\hat{U}_{K}} Z_{K}(\tau) \\
\hat{U}_{K}=\frac{1}{2} \sum_{\xi=a_{ \pm}} \oint_{\xi}\left[y(z) d z-x^{2} d x \oint_{a} \frac{y\left(z^{\prime}\right) d z^{\prime}}{x\left(z^{\prime}\right)^{3}}+\frac{1}{2} \oint_{a_{+}} \frac{d z^{\prime} d z}{2\left(z^{\prime}-z\right)^{2}} \frac{z^{\prime} z-4 S}{y\left(z^{\prime}\right) y(z)} \hat{\Omega}_{K}^{a_{+}}\left(x^{\prime}\right)\right] \hat{\Omega}_{K}^{\xi}(x)
\end{gathered}
$$




\subsection{Comments}

Let us check that the $J_{G}$ and $J_{K}$ are actually local currents corresponding to the global current $\mathbf{J}^{o}$, (2.3) at vicinities of infinity and ramification points. To this end, we compare the corresponding operator product expansions, which are, for the local currents,

$$
\begin{gathered}
\hat{J}_{G}(z) \hat{J}_{G}\left(z^{\prime}\right)=\frac{d z d z^{\prime}}{2\left(z-z^{\prime}\right)^{2}}+\text { regular part } \\
\hat{J}_{K}(z) \hat{J}_{K}\left(z^{\prime}\right)=\frac{\left(x^{2}+x^{\prime 2}\right) d x d x^{\prime}}{\left(x^{2}-x^{\prime 2}\right)^{2}}+\text { regular part }
\end{gathered}
$$

One can immediately check that at the vicinity of infinity, $z \rightarrow \infty$

$$
\frac{d z d z^{\prime}}{2\left(z-z^{\prime}\right)^{2}} \frac{z z^{\prime}-4 S}{y(z) y\left(z^{\prime}\right)}=\frac{d z d z^{\prime}}{2\left(z-z^{\prime}\right)^{2}}+\sum_{i, j=2}^{\infty} \frac{A_{i j} d z d z^{\prime}}{z^{i} z^{\prime j}}
$$

which proves the identity of operator product expansions for $\hat{\mathbf{J}}^{o}$ and $\hat{J}_{G}$ at the vicinity of infinity, while the similar identity for $\hat{\mathbf{J}}^{o}$ and $\hat{J}_{K}$ at the vicinity of ramification points requires some job. Indeed, one needs to prove that

$$
\frac{d z d z^{\prime}}{2\left(z-z^{\prime}\right)^{2}} \frac{z z^{\prime}-4 S}{y(z) y\left(z^{\prime}\right)}=\frac{\left(x^{2}+x^{\prime 2}\right) d x d x^{\prime}}{\left(x^{2}-x^{\prime 2}\right)^{2}}+\sum_{i, j=0}^{\infty} \alpha_{i j} x^{i} x^{\prime j} d x d x^{\prime}
$$

for any local coordinate $x$,

$$
z= \pm 2 \sqrt{S}\left(1+f\left(x_{ \pm}^{2}\right)\right)
$$

with $f(x)$ an arbitrary power series such that $f(0)=0$. Then, one has to prove the two identities

$$
\frac{d z d z^{\prime}}{2\left(z-z^{\prime}\right)^{2}} \frac{z z^{\prime}-4 S}{y(z) y\left(z^{\prime}\right)} \frac{\left(x^{2}-x^{\prime 2}\right)^{2}}{d x d x^{\prime}} \stackrel{x^{2} \rightarrow x^{\prime 2}}{\longrightarrow} 2 x^{2}
$$

and

$$
\frac{\partial}{\partial x}\left(\frac{d z d z^{\prime}}{2\left(z-z^{\prime}\right)^{2}} \frac{z z^{\prime}-4 S}{y(z) y\left(z^{\prime}\right)} \frac{\left(x^{2}-x^{\prime 2}\right)^{2}}{d x d x^{\prime}}\right) \stackrel{x^{2} \rightarrow x^{\prime 2}}{\longrightarrow} 2 x
$$

The first one is quite obvious, the second one requires some (straightforward) calculation. 
$6 \quad Z_{G}(t) \rightarrow Z_{K}\left(\tau_{+}\right) Z_{K}\left(\tau_{-}\right)$

\subsection{Summary}

Let us consider an explicit example of the local coordinate system at the vicinities of ramification points and manifestly relate $Z_{G}$ and $Z_{K}$ in this parametrization. Namely, we choose the local coordinate in accordance with $[18]^{13}$

$$
y(z)^{2}=S\left(e^{x_{ \pm}}-e^{-x_{ \pm}}\right)^{2} \quad z= \pm \sqrt{S}\left(e^{x_{ \pm}}+e^{-x_{ \pm}}\right)
$$

the two signs corresponding to the two ramification points. On the physical plane

$$
d z=y(z) d x
$$

The (1,1)-differentials in this case are

$$
\begin{gathered}
f^{a_{+} a_{+}}\left(z, z^{\prime}\right)=f^{a_{-} a_{-}}\left(z, z^{\prime}\right)=\frac{d z d z^{\prime}}{2\left(z-z^{\prime}\right)^{2}} \frac{z z^{\prime}-4 S}{y(z) y\left(z^{\prime}\right)}-\frac{\left(x_{1}^{2}+x_{2}^{2}\right) d x_{1} d x_{2}}{\left(x_{1}^{2}-x_{2}^{2}\right)^{2}}= \\
=\sum_{i, j=0}^{\infty}\left(C_{++}^{i k}\right) x_{1}^{2 i} x_{2}^{2 j}(2 j+1) d x_{1} d x_{2} \\
f^{a_{+} a_{-}}\left(z, z^{\prime}\right)=f^{a_{-} a_{+}}\left(z, z^{\prime}\right)=\frac{d z d z^{\prime}}{2\left(z-z^{\prime}\right)^{2}} \frac{z z^{\prime}-4 S}{y(z) y\left(z^{\prime}\right)}=\sum_{i, j=0}^{\infty}\left(C_{+-}^{i k}\right) x_{1}^{2 i} x_{2}^{2 j}(2 j+1) d x_{1} d x_{2}
\end{gathered}
$$

where

$$
\sum_{i=0}^{\infty}\left(C_{++}^{i k}\right) x^{2 i}=-\frac{1}{2} \sum_{j=0}^{\infty} \frac{B_{2 j+2 k+2} x^{2 j}}{(2 j+1) !(2 k) !(j+k+1)}
$$

and

$$
\sum_{i=0}^{\infty}\left(C_{+-}^{i k}\right) x^{2 i}=-\frac{1}{2} \sum_{j=0}^{\infty} \frac{B_{2 j+2 k+2} x^{2 j}\left(2^{2 j+2 k+2}-1\right)}{(2 j+1) !(2 k) !(j+k+1)}
$$

Here $B_{2 k}$ are the Bernoulli numbers.

Then local currents are

$$
\hat{J}^{a_{-}}=2 \hat{J}_{K}\left(x_{-} \mid \tau_{-}\right)+\sum_{i j=0}^{\infty} x^{2 i}\left(\left(C_{++}^{i j}\right) \frac{\partial}{\partial \tau_{j}^{-}}-\left(C_{+-}^{i j}\right) \frac{\partial}{\partial \tau_{j}^{+}}\right) d x
$$

and

$$
\hat{J}^{a_{+}}=-2 \hat{J}_{K}\left(x_{+} \mid \tau_{+}\right)+\sum_{i j=0}^{\infty} x^{2 i}\left(-\left(C_{+-}^{i j}\right) \frac{\partial}{\partial \tau_{j}^{-}}+\left(C_{++}^{i j}\right) \frac{\partial}{\partial \tau_{j}^{+}}\right) d x
$$

\footnotetext{
${ }^{13}$ In this choice of local coordinate, the change of time variables can be naturally formulated in terms of an external matrix $\Lambda \equiv e^{\lambda}$, parameterizing the $(T, S)$-time variables

$$
\begin{gathered}
\left(k+\frac{1}{2}\right) T_{k}=\operatorname{Tr} \frac{\Lambda^{2 k}\left(\Lambda^{2}+S\right)}{\left(\Lambda^{2}-S\right)^{2 k+1}}-\delta_{k 0} \\
\left(k+\frac{1}{2}\right) S_{k}=\operatorname{Tr} \frac{\Lambda^{2 k+1}}{\left(\Lambda^{2}-S\right)^{2 k+1}}
\end{gathered}
$$

Then, 5.14 can be represented as

$$
\tau_{k}^{ \pm}=\frac{\sqrt{S}}{(2 k+1) !} \frac{\partial^{2 k}}{\partial \lambda^{2 k}} \operatorname{Tr} \frac{1}{e^{\lambda} \pm \sqrt{S}}
$$

In the same terms, 5.3 can be represented as

$$
t_{k}=\frac{1}{k} \operatorname{Tr} \frac{1}{\left(\Lambda+\sqrt{S} \Lambda^{-1}\right)^{k}}
$$


This means that the conjugation operator in the decomposition formula

$$
Z_{G}(t)=e^{\hat{U}_{G K}} Z_{K}\left(\tau^{+}\right) Z_{K}\left(\tau^{-}\right)
$$

is

$$
\begin{gathered}
\hat{U}_{G K}=U_{G}+\hat{U}_{K}= \\
=U_{G}+\frac{1}{2} \sum_{i, j=\{+,-\}} \oint_{a_{i}} \oint_{a_{j}} f^{a_{i} a_{j}}\left(x_{i}, x_{j}\right) \hat{\Omega}_{K}^{a_{i}}\left(x_{i}\right) \hat{\Omega}_{K}^{a_{j}}\left(x_{j}\right)-\frac{1}{2} \sum_{i=\{+,-\}} \oint_{a_{i}}\left(2 S x^{2}-\frac{y^{2}}{2}\right) \hat{\Omega}_{K}^{a_{i}} d x
\end{gathered}
$$

and, since

$$
y(x)^{2}=S \sum_{k=1}^{\infty} \frac{2^{2 k+1} x^{2 k}}{(2 k) !}
$$

the explicit expression for $\hat{U}_{K}$ coincides with that given in [18,

$$
\begin{gathered}
\hat{U}_{K}=\frac{1}{4} \sum_{i, j=0} \frac{B_{2 i+2 j+2}}{(i+j+1)} \frac{1}{(2 i+1) !(2 j+1) !}\left(\frac{\partial}{\partial \tau_{i}^{+}} \frac{\partial}{\partial \tau_{j}^{+}}+\frac{\partial}{\partial \tau_{i}^{-}} \frac{\partial}{\partial \tau_{j}^{-}}-2\left(2^{2 i+2 j+2}-1\right) \frac{\partial}{\partial \tau_{i}^{+}} \frac{\partial}{\partial \tau_{j}^{-}}\right)+ \\
+\frac{2 S}{g} \sum_{i=2}^{\infty} \frac{2^{2 i-1}}{(2 i+1) !}\left(\frac{\partial}{\partial \tau_{i}^{-}}-\frac{\partial}{\partial \tau_{i}^{+}}\right)
\end{gathered}
$$

\subsection{Comments}

Let us consider here what happens if one does not put $\frac{\partial Z_{G}}{\partial t_{0}}=\frac{S}{g^{2}} Z_{G}$ instead dealing with it as with the differential operator and a partition function $Z_{e G}$ that generalizes the matrix model partition function $Z_{G}$. The global current $\hat{\mathbf{J}}^{o}\left(z, y_{G}\right)$ in the vicinity of the infinity is equivalent to the local current $\hat{J}_{G}(z)-\frac{1}{z} \frac{\partial}{\partial t_{0}}$. To give this current the standard form, one should make the two conjugations: first,

and then

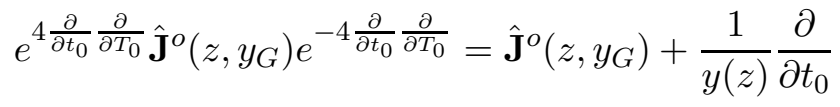

$$
e^{\sum_{k=0}^{\infty} c_{k} T_{k} \frac{\partial}{\partial t_{o}}}\left(\hat{\mathbf{J}}^{o}\left(z, y_{G}\right)+\frac{1}{y(z)} \frac{\partial}{\partial t_{0}}\right) e^{-\sum_{k=0}^{\infty} c_{k} T_{k} \frac{\partial}{\partial t_{o}}}=\hat{\mathbf{J}}^{o}\left(z, y_{G}\right)+\frac{1}{z} \frac{\partial}{\partial t_{0}}
$$

which defines the coefficients $c_{k}$.

Since $\hat{\mathbf{J}}^{o}\left(z, y_{G}\right)=\hat{\nabla}(z)+\ldots$, this conjugation operator can be expressed explicitly

$$
\sum_{k=0}^{\infty} c_{k} T_{k} \frac{\partial}{\partial t_{0}}=\left(T_{-1}-t_{0}\right) \frac{\partial}{\partial t_{0}}
$$

with

$$
T_{-1}=\oint_{\infty} \frac{v(z) d z}{y(z)}
$$

This means that the actual decomposition formula for the Gaussian curve is

$$
e^{-4 \frac{\partial}{\partial t_{0}} \frac{\partial}{\partial T_{0}}} e^{\left(t_{0}-T_{-1}\right) \frac{\partial}{\partial t_{0}}} e^{-\hat{U}_{G}} Z_{e G}=Z\left(t_{0}\right) e^{\hat{U}_{K}} Z_{K}\left(\tau^{+}\right) Z_{K}\left(\tau^{-}\right)
$$

where $Z\left(t_{0}\right)$ is a function (formal series) of $t_{0}$ with genus expansion. This formula describes the decomposition of an arbitrary branch (with genus expansion) of the partition function for the curve $y_{G}(z)$. For the Gaussian solution, $e^{-\hat{U}_{\infty}} Z_{G}$ does not depend on $t_{0}$ and one returns back to

$$
e^{-U_{G}} Z_{G}=e^{\hat{U}_{K}} Z_{K}\left(\tau^{+}\right) Z_{K}\left(\tau^{-}\right)
$$

A nontrivial consequence of this formula is factorizing out the dependence on $t_{0}$. We check this statement explicitly for the perturbative expansion in $t$ in Appendix II and determine first terms of the function $Z\left(t_{0}\right)$ manifestly. 


\section{$7 \quad Z_{A C K M}$ and recovery of Kostov's formula}

\section{1 $Z_{G}(t) \rightarrow Z_{K}\left(\tau_{+}\right) Z_{K}\left(\tau_{-}\right)$in the non-symmetric case. Summary}

In this section we consider the non-symmetric curve

$$
y^{2}(z)=\left(z-a_{+}\right)\left(z-a_{-}\right)
$$

with constant $a_{ \pm}$. Now we, following the line of s.5-6, repeat all the calculation for non-symmetric case and for generically chosen coordinates in the vicinities of the two ramification points (i.e. for the two corresponding local parameters chosen independently). The global current now is the following

$$
\begin{gathered}
\hat{\mathbf{J}}^{o}(z)=\frac{1}{2} \sum_{k=0}^{\infty}\left(k+\frac{1}{2}\right)\left(\left(z-a_{+}\right)\left(T_{+}^{k}+\frac{2 S \delta_{k, 0}}{a_{-}-a_{+}}\right)+\left(z-a_{-}\right)\left(T_{-}^{k}-\frac{2 S \delta_{k 0}}{a_{-}-a_{+}}\right)\right) y^{2 k-1} d z+g^{2} \\
\sum_{k=0}\left(\left(z-a_{+}\right) \frac{\partial}{\partial \tilde{T}_{+}^{k}}+\left(z-a_{-}\right) \frac{\partial}{\partial \tilde{T}_{-}^{k}}\right) y^{-2 k-3} d z
\end{gathered}
$$

where

$$
\begin{gathered}
\frac{\partial}{\partial \tilde{T}_{+}^{k}}=\frac{\partial}{\partial T_{+}^{k}}+\frac{\partial}{\partial T_{-}^{k}}+\frac{4(k+1)}{(2 k+3)\left(a_{+}-a_{-}\right)^{2}}\left(\frac{\partial}{\partial T_{+}^{k-1}}-\frac{\partial}{\partial T_{-}^{k-1}}\right) \\
\frac{\partial}{\partial \tilde{T}_{-}^{k}}=\frac{\partial}{\partial T_{-}^{k}}+\frac{\partial}{\partial T_{+}^{k}}+\frac{4(k+1)}{(2 k+3)\left(a_{+}-a_{-}\right)^{2}}\left(\frac{\partial}{\partial T_{-}^{k-1}}-\frac{\partial}{\partial T_{+}^{k-1}}\right)
\end{gathered}
$$

and we deal with $S$ as a parameter independent on $a_{ \pm}$. Nearby infinity the global current is equivalent to the corresponding local current

$$
\hat{J}_{G}(z)=\frac{1}{2} \sum_{k=0} k t_{k} z^{k-1} d z+\frac{S d z}{z}+\sum_{k=1} \frac{d z}{z^{k+1}} \frac{\partial}{\partial t_{k}}
$$

By definition, one has

$$
\begin{aligned}
& T_{+}^{k}=\frac{1}{\left(k+\frac{1}{2}\right)\left(a_{-}-a_{+}\right)} \oint_{\infty} \frac{\left(z-a_{+}\right) d v(z)}{y^{2 k+1}} \\
& T_{-}^{k}=\frac{1}{\left(k+\frac{1}{2}\right)\left(a_{+}-a_{-}\right)} \oint_{\infty} \frac{\left(z-a_{-}\right) d v(z)}{y^{2 k+1}}
\end{aligned}
$$

The conjugation operator, as in the symmetric case, is

$$
U_{G}=\frac{1}{2 g^{2}} \oint_{\infty} \oint_{\infty} \rho^{(0 \mid 2)}\left(z_{1}, z_{2}\right) v^{\Sigma}\left(z_{1}\right) v^{\Sigma}\left(z_{2}\right)+\frac{S}{g^{2}} \oint\left(\frac{1}{y}-\frac{1}{z}\right) v^{\Sigma}(z) d z+f\left(S, a_{ \pm}\right)
$$

where

$$
\rho^{(0 \mid 2)}\left(z_{1}, z_{2}\right)=\frac{1}{2\left(z_{1}-z_{2}\right)^{2}}\left(\frac{z_{1} z_{2}-\left(a_{+}+a_{-}\right)\left(z_{1}+z_{2}\right) / 2+a_{+} a_{-}}{y\left(z_{1}\right) y\left(z_{2}\right)}-1\right)
$$

and $f\left(S, a_{ \pm}\right)$is given in (7.12). Like above,

$$
\tau_{ \pm}^{k}=\frac{1}{(k+1 / 2)} \oint_{a_{ \pm}} \frac{d v(z)}{x_{ \pm}^{2 k+1}}
$$

and the operator $\hat{U}_{K}$ is now

$$
\hat{U}_{K}=8 g^{2} \sum_{i, j= \pm} \sum_{k, m=0}^{\infty} A_{i j}^{k m} \frac{\partial^{2}}{\partial \tau_{k}^{i} \partial \tau_{m}^{j}}
$$


where

$$
\begin{aligned}
A_{++}^{k m} & =\oint_{a_{+}} \oint_{a_{+}}\left(\frac{d z_{1} d z_{2}}{2\left(z_{1}-z_{2}\right)^{2}}\left(\frac{z_{1} z_{2}-\left(a_{+}+a_{-}\right)\left(z_{1}+z_{2}\right) / 2+a_{+} a_{-}}{y\left(z_{1}\right) y\left(z_{2}\right)}\right)\right) \frac{1}{(2 k+1)(2 m+1) x_{1}^{2 k+1} x_{2}^{2 m+1}} \\
A_{--}^{k m} & =\oint_{a_{-}} \oint_{a_{-}}\left(\frac{d z_{1} d z_{2}}{2\left(z_{1}-z_{2}\right)^{2}}\left(\frac{z_{1} z_{2}-\left(a_{+}+a_{-}\right)\left(z_{1}+z_{2}\right) / 2+a_{+} a_{-}}{y\left(z_{1}\right) y\left(z_{2}\right)}\right)\right) \frac{1}{(2 k+1)(2 m+1) x_{1}^{2 k+1} x_{2}^{2 m+1}} \\
A_{+-}^{k m} & \left.=\oint_{a_{+}} \oint_{a_{-}} \frac{d z_{1} d z_{2}}{2\left(z_{1}-z_{2}\right)^{2}}\left(\frac{z_{1} z_{2}-\left(a_{+}+a_{-}\right)\left(z_{1}+z_{2}\right) / 2+a_{+} a_{-}}{y\left(z_{1}\right) y\left(z_{2}\right)}\right) \frac{1}{(2 k+1)(2 m+1) x_{1}^{2 k+1} x_{2}^{2 m+1}}\right) \frac{1}{(2 k+1)(2 m+1) x_{1}^{2 k+1} x_{2}^{2 m+1}} \\
A_{-+}^{k m} & =\oint_{a_{-}} \oint_{a_{+}} \frac{d z_{1} d z_{2}}{2\left(z_{1}-z_{2}\right)^{2}}\left(\frac{\left.z_{1} z_{2}-\left(a_{+}+a_{-}\right)\left(z_{1}+z_{2}\right) / 2+a_{+}\right)}{y\left(z_{-}\right)}\right)
\end{aligned}
$$

and

$$
Z_{G}\left(t, g^{2}\right)=e^{\hat{U}_{G}} e^{\hat{U}_{K}} Z_{K}\left(\tau^{+}, 4 g^{2}\right) Z_{K}\left(\tau^{-}, 4 g^{2}\right)
$$

As usual, this formula is defined up to an arbitrary factor $f\left(S, a_{ \pm}\right)$. Below we prove that, in the present case, this factor is equal to

$$
f\left(S, a_{ \pm}\right)=-\left(\frac{S^{2}}{g^{2}}+\frac{1}{8}\right) \log \left(a_{+}-a_{-}\right)
$$

\subsection{Kostov's formula and ACKM polynomial decomposition}

Now using the decomposition formula (7.11), we construct a polynomial (moment) representation of the Gaussian partition function $Z_{G}$. Our strategy is as follows. First of all, we make the change of variables as in (3.3) with some functions $G_{ \pm}$and zero $u$ and then, after calculating all the derivatives w.r.t. time variables in (7.11), fix $G_{ \pm}$and $a_{ \pm}$to be specific functions of times so that the r.h.s. of (7.11) becomes a polynomial of moment variables (2.27) at each genus. This is an allowed procedure, since the r.h.s. of (7.11) does not really depend on $a_{ \pm}$! Therefore, one may put them to be any functions of times.

Thus, we start from the transformation of times (3.3) with $u=0$ so that

$$
Z_{K}\left(\tau_{0}, \tau_{1}, \tau_{2}, \ldots, g^{2}\right)=G^{-\frac{1}{24}} Z_{K}\left(\tau_{0} G^{-1 / 3}, \tau_{1} G^{-1}, \tau_{2} G^{-5 / 3}, \ldots, g^{2}\right)
$$

and

$$
\begin{aligned}
Z_{G}=e^{U_{G}-\frac{1}{24} \log \left(G_{+} G_{-}\right)} e^{\hat{U}_{K}} Z_{K}\left(\tau_{0}^{+} G_{+}^{-1 / 3}, \tau_{1}^{+} G_{+}^{-1 / 3}, \tau_{2}^{+} G_{+}^{-5 / 3}, \ldots, 4 g^{2}\right) \times \\
\times Z_{K}\left(\tau_{0}^{-} G_{-}^{-1 / 3}, \tau_{1}^{-} G_{-}^{-1}, \tau_{2}^{-} G_{-}^{-5 / 3}, \ldots, 4 g^{2}\right)
\end{aligned}
$$

Now one should achieve $\tilde{\tau}_{0,1}^{\Sigma \pm}=0$ so that $\log Z_{G}$ become a finite degree polynomial at each genus in ACKM moment variables defined as

$$
\begin{gathered}
t_{k}^{-}=\frac{1}{2} \oint_{A_{+}, A_{-}} \frac{d v}{y(z)\left(z-A_{+}\right)^{k}}=-\frac{1}{2 \sqrt{d}} \sum_{m=0}^{k-1} \frac{\Gamma(1 / 2)(k-m+1 / 2)}{\Gamma(m+1) \Gamma(1 / 2-m)} \frac{\tau_{k-m}^{+}}{d^{m}} \\
t_{k}^{+}=\frac{1}{2} \oint_{A_{+}, A_{-}} \frac{d v}{y(z)\left(z-A_{-}\right)^{k}}=(-1)^{k+1} \frac{1}{2 \sqrt{d}} \sum_{m=0}^{k-1} \frac{\Gamma(1 / 2)(k-m+1 / 2)}{\Gamma(m+1) \Gamma(1 / 2-m)} \frac{\tau_{k-m}^{-}}{d^{m}}
\end{gathered}
$$

where $d \equiv A_{+}-A_{-}$. To this end, after the action of the differential conjugation operator, one should consider

1) special $a_{ \pm}=A_{ \pm}$, namely, those satisfying the condition

$$
T_{ \pm}^{0} \pm \frac{2 S}{A_{-}-A_{+}}=0
$$

This fixes $\tilde{\tau}_{0}^{\Sigma \pm}=0$. Note that such $A_{ \pm}$coincide with appropriate ramification points in [42] 
2) $\quad$ special $G_{ \pm}$such that $\left(\tau_{1}^{\Sigma \pm}-\frac{2}{3}\right) G_{ \pm}^{-1}+\frac{2}{3}=0$. This fixes $\tilde{\tau}_{1}^{\Sigma \pm}=0$.

Finally one gets

$$
Z_{G}\left(t^{ \pm}, g^{2}\right)=\left.e^{U_{G}-\frac{1}{24} \log \left(\tau_{1}^{+} \tau_{1}^{-}\right)} e^{\hat{U}_{K}\left(a_{ \pm}\right)} Z_{K}\left(\tau_{k}^{+} G_{+}^{\frac{-2 k+1}{3}}, 4 g^{2}\right) Z_{K}\left(\tau_{k}^{-} G_{-}^{\frac{-2 k+1}{3}}, 4 g^{2}\right)\right|_{a_{ \pm}=A_{ \pm}, \tau_{0}^{\Sigma \pm}=0, G_{ \pm}=1-\frac{3}{2} \tau_{1}^{\Sigma \pm}}
$$

This formula was first suggested by I.Kostov in the second paper of [19. ${ }^{14}$ Here we provided its proof and applications. In particular, we demonstrate how to use this formula to obtain effectively explicit formulas for the free energy in the example of the genus 2 free energy in Appendix I.

\subsection{Comments}

It remains to prove that the r.h.s. of (7.11) does not depend on $a_{+}$and $a_{-}$so that they can be put equal to any functions of times $A_{ \pm}$(after taking all the time derivatives). For simplicity, we consider only dependence on $a_{-}$:

$$
\begin{gathered}
\frac{\partial}{\partial a_{-}} e^{U_{G}} e^{\hat{U}_{K}} Z_{K}\left(\tau^{-}\right) Z_{K}\left(\tau^{+}\right)=e^{U_{G}} \frac{\partial U_{G}}{\partial a_{-}} e^{\hat{U}_{K}} Z_{K}\left(\tau^{-}\right) Z_{K}\left(\tau^{+}\right)+ \\
+e^{U_{G}} e^{\hat{U}_{K}}\left(\frac{\partial \hat{U}_{K}}{\partial a_{-}}+\sum_{k=0}^{\infty} \frac{\partial \tau_{k}^{+}}{\partial a_{-}} \frac{\partial}{\partial \tau_{k}^{+}}+\sum_{k=0}^{\infty} \frac{\partial \tau_{k}^{-}}{\partial a_{-}} \frac{\partial}{\partial \tau_{k}^{-}}\right) Z_{K}\left(\tau^{-}\right) Z_{K}\left(\tau^{+}\right)= \\
=e^{U_{G}} e^{\hat{U}_{K}}\left(\partial_{-} U_{G}+\left[\partial_{-} U_{G}, \hat{U}_{K}\right]+\frac{1}{2}\left[\left[\partial_{-} U_{G}, \hat{U}_{K}\right], \hat{U}_{K}\right]+\right. \\
\left.+\partial_{-} \hat{U}_{K}+\sum_{k=0}^{\infty} \frac{\partial \tau_{k}^{+}}{\partial a_{-}} \frac{\partial}{\partial \tau_{k}^{+}}+\sum_{k=0}^{\infty} \frac{\partial \tau_{k}^{-}}{\partial a_{-}} \frac{\partial}{\partial \tau_{k}^{-}}\right) Z_{K}\left(\tau^{-}\right) Z_{K}\left(\tau^{+}\right)
\end{gathered}
$$

The expression in the brackets in the last two lines is a second order differential operator. We prove that it is equal to $-L_{-1}^{K}\left(\tau^{-}\right)$:

$$
\begin{gathered}
\partial_{-} \rho^{(0 \mid 2)}\left(z_{1}, z_{2}\right)=-\frac{d}{8 y\left(z_{1}\right) y\left(z_{2}\right)\left(z_{1}-a_{-}\right)\left(z_{2}-a_{-}\right)} \\
\partial_{-} U_{G}=-\frac{1}{16 g^{2}}\left(\oint_{\infty} \frac{v d z}{y(z)\left(z-a_{-}\right)}\right)^{2}+\frac{S}{2 g^{2}} \oint_{\infty} \frac{v d z}{y(z)\left(z-a_{-}\right)}+\partial_{-} f=-\frac{\left(\tau_{0}^{-}\right)^{2}}{64 g^{2}}+\frac{S^{2}}{g^{2} d}+\partial_{-} f \\
\hat{\Psi}=\oint_{a_{+}} \frac{1}{y(z)\left(z-a_{-}\right)}+\oint_{a_{-}} \frac{\partial}{y(z)\left(z-a_{-}\right)}=-\frac{4}{\sqrt{d}} \sum_{k=0}^{\infty}\left(\alpha_{k}^{-} \frac{\partial}{\partial \tau_{k}^{+}}+\beta_{k}^{-} \frac{\partial}{\partial \tau_{k}^{-}}\right) \\
\left.\hat{\Psi}=-d^{-\frac{3}{2}} \frac{\partial}{\partial \tau_{0}^{-}}+2 d^{-\frac{3}{2}} \frac{\partial}{\partial \tau_{0}^{+}}+\ldots ; \tau_{0}^{-}\right]=-d^{-\frac{3}{2}} \\
{\left[\hat{U}_{K}, \tau_{0}^{-}\right]=8 g^{2} \sqrt{d} \hat{\Psi}} \\
f_{k}^{ \pm}=\frac{1}{\sqrt{d}}\left(\oint_{a_{-}}+\oint_{a_{+}}\right) \frac{( \pm 1)^{k+1} y(z) d v}{\left(z-a_{ \pm}\right)^{k+1}} \\
\tau_{ \pm}^{k}=\frac{8}{2 k+1} \sum_{m=1}^{k+1} \frac{\Gamma(2 m-1)(-1)^{m+1} f_{k-m+1}^{ \pm}}{\Gamma(m)^{2} 4^{m} d^{m-1}} \\
\frac{\partial-f_{k}^{-}}{2 k}=\frac{1}{2 d} f_{k}^{-}-\left(k+\frac{1}{2}\right) f_{k+1}^{-}
\end{gathered}
$$

\footnotetext{
${ }^{14}$ It seems to us that in formula (7.24) of this paper instead of $\frac{1}{r r^{\prime}}$ one should write $\frac{2}{(2 r) ! !} \frac{2}{\left(2 r^{\prime}\right) ! !}$.
} 


$$
\begin{aligned}
& \partial_{-} \tau_{k}^{-}=-\left(k+\frac{3}{2}\right) \tau_{k+1}^{-}+\frac{2}{2 k+1} \frac{\Gamma(2 k+3)(-1)^{k}}{\Gamma(k+2)^{2} 4^{k+2} d^{k+1}} \tau_{0}^{-}=-\left(k+\frac{3}{2}\right) \tau_{k+1}^{-}+\beta_{k}^{-} \tau_{0}^{-} \\
& \partial_{-} \tau_{k}^{+}=-\frac{\Gamma(2 k+1)(-1)^{k}}{\Gamma(k+1)^{2} 4^{k} d^{k+1}} \frac{\tau_{0}^{-}}{2}=\alpha_{k}^{-} \tau_{0}^{-} \\
& \sum_{k=0}^{\infty} \frac{\partial \tau_{k}^{+}}{\partial a_{-}} \frac{\partial}{\partial \tau_{k}^{+}}+\sum_{k=0}^{\infty} \frac{\partial \tau_{k}^{-}}{\partial a_{-}} \frac{\partial}{\partial \tau_{k}^{-}}=-\sum_{k=0}^{\infty}\left(k+\frac{3}{2}\right) \tau_{k+1}^{-} \frac{\partial}{\partial \tau_{k}^{-}}-\frac{\sqrt{d}}{4} \tau_{0}^{-} \hat{\Psi} \\
& {\left[\partial_{-}, \hat{\Omega}^{+}(x)\right]=0 ; \quad\left[\partial_{-}, \hat{\Omega}^{-}(x)\right]=-\frac{\partial}{\partial x^{2}} \hat{\Omega}^{-}(x)+\frac{1}{x} \frac{\sqrt{d}}{4} \hat{\Psi}} \\
& \partial_{-} \hat{U}_{K}=8 g^{2} \partial_{-}\left(4 \oint_{a_{+}} \oint_{a_{+}} \rho^{(0 \mid 2)}\left(a_{+}+x_{1}^{2}, a_{+}+x_{2}^{2}\right) x_{1} x_{2} d x_{1} d x_{2} \hat{\Omega}_{+}\left(x_{1}\right) \hat{\Omega}_{+}\left(x_{2}\right)\right. \\
& -8 \oint_{a_{+}} \oint_{a_{-}} \rho^{(0 \mid 2)}\left(a_{+}+x_{1}^{2}, a_{-}-x_{2}^{2}\right) x_{1} x_{2} d x_{1} d x_{2} \hat{\Omega}_{+}\left(x_{1}\right) \hat{\Omega}_{-}\left(x_{2}\right)+ \\
& \left.+4 \oint_{a_{-}} \oint_{a_{-}} \rho^{(0 \mid 2)}\left(a_{-}-x_{1}^{2}, a_{-}-x_{2}^{2}\right) x_{1} x_{2} d x_{1} d x_{2} \hat{\Omega}_{-}\left(x_{1}\right) \hat{\Omega}_{-}\left(x_{2}\right)\right) \\
& \partial_{-} \oint_{a_{+}} \oint_{a_{+}} \rho^{(0 \mid 2)}\left(a_{+}+x_{1}^{2}, a_{+}+x_{2}^{2}\right) x_{1} x_{2} d x_{1} d x_{2} \hat{\Omega}_{+}\left(x_{1}\right) \hat{\Omega}_{+}\left(x_{2}\right)= \\
& =\oint_{a_{+}} \oint_{a_{+}}\left(\partial_{-} \rho^{(0 \mid 2)}\left(a_{+}+x_{1}^{2}, a_{+}+x_{2}^{2}\right)\right) x_{1} x_{2} d x_{1} d x_{2} \hat{\Omega}_{+}\left(x_{1}\right) \hat{\Omega}_{+}\left(x_{2}\right)=-\frac{d}{8}\left(\oint_{a_{+}} \frac{\hat{\Omega}^{+}(x) x d x}{\left(d+x^{2}\right) y\left(a_{+}+x^{2}\right)}\right)^{2} \\
& \partial_{-} \oint_{a_{+}} \oint_{a_{-}} \rho^{(0 \mid 2)}\left(a_{+}+x_{1}^{2}, a_{-}-x_{2}^{2}\right) x_{1} x_{2} d x_{1} d x_{2} \hat{\Omega}_{+}\left(x_{1}\right) \hat{\Omega}_{-}\left(x_{2}\right)= \\
& =\oint_{a_{+}} \oint_{a_{-}}\left(\left(\partial_{-}-\frac{\partial}{\partial x_{2}^{2}}\right) \rho^{(0 \mid 2)}\left(a_{+}+x_{1}^{2}, a_{-}-x_{2}^{2}\right)\right) x_{1} x_{2} d x_{1} d x_{2} \hat{\Omega}_{+}\left(x_{1}\right) \hat{\Omega}_{-}\left(x_{2}\right)+ \\
& +\oint_{a_{+}} \oint_{a_{-}} \rho^{(0 \mid 2)}\left(a_{+}+x_{1}^{2}, a_{-}-x_{2}^{2}\right) x_{1} x_{2} d x_{1} d x_{2} \hat{\Omega}_{+}\left(x_{1}\right) \partial_{-} \hat{\Omega}_{-}\left(x_{2}\right)= \\
& =\frac{d}{8}\left(\oint_{a_{+}} \frac{\hat{\Omega}^{+}(x) x d x}{\left(d+x^{2}\right) y\left(a_{+}+x^{2}\right)}\right)\left(\oint_{a_{-}} \frac{\hat{\Omega}^{-}(x) d x}{x y\left(a_{-}-x^{2}\right)}\right)-\frac{d}{16} \oint_{a_{+}} \frac{\hat{\Omega}^{+}(x) x d x}{\left(d+x^{2}\right) y\left(a_{+}+x^{2}\right)} \hat{\Psi} \\
& \partial_{-} \oint_{a_{-}} \oint_{a_{-}} \rho^{(0 \mid 2)}\left(a_{-}-x_{1}^{2}, a_{-}-x_{2}^{2}\right) x_{1} x_{2} d x_{1} d x_{2} \hat{\Omega}_{-}\left(x_{1}\right) \hat{\Omega}_{-}\left(x_{2}\right)= \\
& =-\frac{d}{8}\left(\oint_{a_{-}} \frac{\hat{\Omega}^{-}(x) d x}{x y\left(a_{-}-x^{2}\right)}\right)^{2}+\frac{d}{8} \oint_{a_{-}} \frac{\hat{\Omega}^{-}(x) d x}{x y\left(a_{-}-x^{2}\right)} \hat{\Psi} \\
& \partial_{-} \hat{U}_{K}=8 g^{2}\left(-\frac{d}{2}\left(\oint_{a_{+}} \frac{\hat{\Omega}^{+}(x) x d x}{\left(d+x^{2}\right) y\left(a_{+}+x^{2}\right)}+\oint_{a_{-}} \frac{\hat{\Omega}^{-}(x) d x}{x y\left(a_{-}-x^{2}\right)}\right)^{2}+\right. \\
& \left.+\frac{d}{2}\left(\oint_{a_{+}} \frac{\hat{\Omega}^{+}(x) x d x}{\left(d+x^{2}\right) y\left(a_{+}+x^{2}\right)}+\oint_{a_{-}} \frac{\hat{\Omega}^{-}(x) d x}{x y\left(a_{-}-x^{2}\right)}\right) \hat{\Psi}\right)=g^{2} d \hat{\Psi}^{2} \\
& {\left[\partial_{-} U_{G}, \hat{U}_{K}\right]=-\frac{1}{8 d}+\frac{\sqrt{d}}{4} \tau_{0}^{-} \hat{\Psi}} \\
& \frac{1}{2}\left[\left[\partial_{-} U_{G}, \hat{U}_{K}\right], \hat{U}_{K}\right]=-g^{2} d \hat{\Psi}^{2}
\end{aligned}
$$

Summing up expressions (7.20), (7.30), (7.36), (7.37) and (7.38), one finally gets that the r.h.s. of (7.18) is $-e^{U_{G}} e^{\hat{U}_{K}} L_{-1}^{K}\left(\tau^{-}\right) Z_{K}\left(\tau^{-}\right) Z_{K}\left(\tau^{+}\right)$plus an additional piece that is removed by the proper choice of an arbitrary multiplicative factor depending only on $S$ and $a_{ \pm}$:

$$
U_{G}=\ldots-\frac{S^{2}}{g^{2}} \log d-\frac{1}{8} \log d
$$




\section{Decomposition formula for complex matrix model}

To illustrate our generic method in a slightly different situation, in this section we construct a decomposition formula for the $\mathbf{Z}(T)$ projection of the global partition function and consider current on the sphere with 6 singular points: two infinities, two ramification points and two zeros. This decomposition formula involves the complex matrix model, the Kontsevich one and also $\tilde{Z}(\mathcal{T})$ : $Z_{C}(\mathfrak{t}) \longrightarrow Z_{K}(\tau) \tilde{Z}_{K}(\mathcal{T})$.

\subsection{Summary}

The global current in this case is

$$
\begin{gathered}
\hat{\mathbf{J}}=\frac{\hat{\mathbf{J}}^{o}(z)+\hat{\mathbf{J}}^{o}(-z)}{2}=\hat{\mathbf{J}}^{o}(z \mid T, 0) \\
f_{\hat{\mathbf{J}}}\left(z, z^{\prime} \mid g^{2}\right)=g^{2} \frac{\left(y^{2} y^{\prime 2}+2 S\left(y^{2}+y^{\prime 2}\right)\right) d z d z^{\prime}}{\left(y^{2}-y^{\prime 2}\right)^{2} y y^{\prime}}
\end{gathered}
$$

This global current is equivalent to the complex model current in the vicinity of infinities and to the Kontsevich current in the vicinity of ramification points and zeros. The proof is similar to s.5.3):

1) $\infty_{ \pm}$The equivalence of currents follows from the simple relations

$$
\begin{gathered}
f_{\hat{\mathbf{J}}}\left(z, z^{\prime} \mid g^{2}\right) \frac{\left(z^{2}-z^{\prime 2}\right)^{2}}{d z d z^{\prime}} \longrightarrow g^{2} z^{2} \\
\frac{\partial}{\partial z}\left(f_{\hat{\mathbf{J}}}\left(z, z^{\prime} \mid g^{2}\right) \frac{\left(z^{2}-z^{\prime 2}\right)^{2}}{d z d z^{\prime}}\right) \longrightarrow g^{2} z
\end{gathered}
$$

with the times being

$$
\mathfrak{t}_{k}=\frac{2}{k} \oint_{\infty} z^{-2 k} \hat{\mathbf{J}}
$$

2) $\pm 2 \sqrt{S}$ We use the local coordinate $x$ such as

$$
z=2 \sqrt{S}\left(1+\frac{x^{2}}{2(4 S)^{\frac{2}{3}}}\right)
$$

It is convenient, because

$$
y(z) d z=\left(x^{2}+O\left(x^{3}\right)\right) d x
$$

Similarly to s.5.3,

$$
\begin{gathered}
f_{\hat{\mathbf{J}}}\left(z, z^{\prime} \mid g^{2}\right) \frac{\left(x^{2}-x^{\prime 2}\right)^{2}}{d x d x^{\prime}} \longrightarrow g^{2} x^{2} \\
\frac{\partial}{\partial x}\left(f_{\hat{\mathbf{J}}}\left(z, z^{\prime} \mid g^{2}\right) \frac{\left(x^{2}-x^{\prime 2}\right)^{2}}{d x d x^{\prime}}\right) \longrightarrow g^{2} x
\end{gathered}
$$

and the times are

$$
\tau_{k}=\frac{4}{2 k+1} \oint_{2 \sqrt{S}} x^{-2 k-1} \hat{\mathbf{J}}
$$


3) $0_{ \pm}$We use $z$ as a local coordinate

$$
\begin{gathered}
f_{\hat{\mathbf{J}}}\left(z, z^{\prime} \mid g^{2}\right) \frac{\left(z^{2}-z^{\prime 2}\right)^{2}}{d z d z^{\prime}} \longrightarrow g^{2} z^{2} \\
\frac{\partial}{\partial z}\left(f_{\hat{\mathbf{J}}}\left(z, z^{\prime} \mid g^{2}\right) \frac{\left(z^{2}-z^{\prime 2}\right)^{2}}{d z d z^{\prime}}\right) \longrightarrow g^{2} z
\end{gathered}
$$

the times being

$$
\mathcal{T}_{k}=\frac{4}{2 k+1} \oint_{0} z^{-2 k-1} \hat{\mathbf{J}}
$$

With the shift

$$
\Delta \hat{\mathbf{J}}(z)=-\frac{y(z) d z}{2}
$$

there is an equivalence

$$
\begin{gathered}
\hat{\mathbf{J}}\left(z \mid g^{2}\right) \stackrel{z \rightarrow \infty}{\sim} \hat{J}_{C}\left(z \mid 2 g^{2}\right) \\
\hat{\mathbf{J}}\left(z \mid g^{2}\right) \stackrel{z \rightarrow \pm 2 \sqrt{S}}{\sim} \hat{J}_{K}\left(c \mid 2 g^{2}\right) \\
\hat{\mathbf{J}}\left(z \mid g^{2}\right) \stackrel{z \rightarrow 0_{ \pm}}{\sim} \hat{J}_{K}\left(z \mid 2 g^{2}\right)
\end{gathered}
$$

in the sense of (2.43) such that

$$
\begin{aligned}
& \alpha_{\infty}=\alpha_{a}=\alpha_{0}=1 \\
& \beta_{\infty}=\beta_{a}=\beta_{0}=2
\end{aligned}
$$

According to (2.45), the conjugation operator ${ }^{15}$ is

$$
\begin{aligned}
& V_{\infty}\left(2 g^{2}\right)=V_{C}=\frac{2}{g^{4}} \oint_{\infty} \oint_{\infty}\left(f_{\hat{\mathbf{J}}}\left(z, z^{\prime} \mid g^{2}\right)-f_{C}\left(z, z^{\prime} \mid 2 g^{2}\right)\right) \hat{\Omega}^{C}\left(z \mid 2 g^{2}\right) \hat{\Omega}^{C}\left(z^{\prime} \mid 2 g^{2}\right)+ \\
&+\oint_{\infty} k^{\infty}\left(z \mid 2 g^{2}\right) \hat{\Omega}^{C}\left(z \mid 2 g^{2}\right)
\end{aligned}
$$

with

$$
\begin{gathered}
f_{\hat{\mathbf{J}}}\left(z, z^{\prime} \mid g^{2}\right)-f_{C}\left(z, z^{\prime} \mid 2 g^{2}\right)=2 g^{2} \rho_{C}^{(0 \mid 2)}\left(z, z^{\prime}\right) \\
k^{\infty}\left(z \mid 2 g^{2}\right)=\frac{(z-y(z)) d z}{g^{2}}
\end{gathered}
$$

and the two-point resolvent $\rho_{C}^{(0 \mid 2)}\left(z, z^{\prime}\right)$ here is explicitly given by (A1.18). Since

$$
\hat{\Omega}^{C}\left(z \mid 2 g^{2}\right)=\frac{v_{C}(z)}{4}+\ldots
$$

the conjugation operator at infinity is

$$
V_{\infty}\left(2 g^{2}\right)=V_{C}=\frac{1}{2 ! 2 g^{2}} \oint_{\infty} \oint_{\infty} \rho_{C}^{(0 \mid 2)}\left(z, z^{\prime}\right) v_{C}(z) v_{C}\left(z^{\prime}\right)+\frac{1}{2 g^{2}} \oint_{\infty} \rho_{C}^{(0 \mid 1)}(z) v_{C}(z)
$$

with one-point resolvent $\rho_{C}^{(0 \mid 1)}(z)$ given at (A1.16). The second conjugation operator consist of three parts

$$
\hat{V}_{\text {ram }}=\hat{V}_{a a}+\hat{V}_{a 0}+\hat{V}_{00}
$$

\footnotetext{
${ }^{15}$ In order to differ from the case of four singular points, we denote conjugation operators of this section through $V$ instead of $U$.
} 
and

$$
\begin{gathered}
\hat{V}_{a a}=\frac{2}{g^{4}} \oint_{2 \sqrt{S}} \oint_{2 \sqrt{S}}\left(f_{\hat{\mathbf{J}}}\left(z, z^{\prime} \mid g^{2}\right)-f_{K}\left(x, x^{\prime} \mid 2 g^{2}\right)\right) \hat{\Omega}^{K}\left(x \mid 2 g^{2}\right) \hat{\Omega}^{K}\left(x \mid 2 g^{2}\right)+\oint_{2 \sqrt{S}} k^{a}\left(x \mid 2 g^{2}\right) \hat{\Omega}^{K}\left(x \mid 2 g^{2}\right) \\
\hat{V}_{a 0}=\frac{2}{g^{4}} \oint_{2 \sqrt{S}} \oint_{0} f_{\hat{\mathbf{J}}}\left(z, z^{\prime} \mid g^{2}\right) \hat{\Omega}^{K}\left(x \mid 2 g^{2}\right) \hat{\Omega}^{K}\left(z \mid 2 g^{2}\right) \\
\hat{V}_{00}=\frac{2}{g^{4}} \oint_{0} \oint_{0}\left(f_{\hat{\mathbf{J}}}\left(z, z^{\prime} \mid g^{2}\right)-f_{K}\left(z, z^{\prime} \mid 2 g^{2}\right)\right) \hat{\Omega}^{K}\left(z \mid 2 g^{2}\right) \hat{\Omega}^{K}\left(z^{\prime} \mid 2 g^{2}\right)+\oint_{0} k^{0}\left(z \mid 2 g^{2}\right) \hat{\Omega}^{K}\left(z \mid 2 g^{2}\right)
\end{gathered}
$$

where

$$
\begin{aligned}
k^{a}\left(x \mid 2 g^{2}\right) & =\frac{x^{2} d x-y d z}{g^{2}} \\
k^{0}\left(z \mid 2 g^{2}\right) & =\frac{z d z-y d z}{g^{2}}
\end{aligned}
$$

Therefore,

$$
e^{-V_{\infty}\left(2 g^{2}\right)} Z_{C}\left(\mathfrak{t} \mid 2 g^{2}\right)=e^{\hat{V}_{r a m}\left(2 g^{2}\right)} Z_{K}\left(\tau \mid 2 g^{2}\right) \tilde{Z}_{K}\left(\mathrm{t} \mid 2 g^{2}\right)
$$

After the change

$$
2 g^{2} \rightarrow g^{2}
$$

one finally gets

$$
e^{-V_{\infty}\left(g^{2}\right)} Z_{C}\left(\mathfrak{t} \mid g^{2}\right)=e^{\hat{V}_{r a m}\left(g^{2}\right)} Z_{K}\left(\tau \mid g^{2}\right) \tilde{Z}_{K}\left(\mathrm{t} \mid g^{2}\right)
$$

where one should put $c=2 \sqrt{-4 S}$ in the definition of $\tilde{Z}_{K}$.

\subsection{Comments on moment variables for $Z_{C}$}

Consider the coordinate transformation

$$
\tilde{z}=a \sqrt{z^{2}-u}
$$

and look for an identity similar to (4.8)

$$
Z_{C}\left(\mathfrak{t}_{2 k}\right)=e^{U_{C}} Z_{C}\left(\tilde{\mathfrak{t}}_{k}\right)
$$

This time we work with the current (2.17)

$$
\hat{J}_{C}(z)=\frac{1}{2} \sum_{k=0} k \mathfrak{t}_{k} z^{2 k-1} d z+\frac{S}{z}+g^{2} \sum_{k=1} \frac{1}{z^{2 k+1}} \frac{\partial}{\partial \mathfrak{t}_{k}}
$$

which invariance (up to conjugation) gives rise to the change of time variables

$$
k \tilde{\mathfrak{t}}_{k}=\oint_{\infty} \frac{d v(z)}{\tilde{z}^{2 k}}=\frac{1}{a^{2 k}} \sum_{n=k} \mathfrak{t}_{2} \frac{n ! u^{n-k}}{(k-1) !(n-k) !}
$$

and the corresponding conjugation operator is

$$
e^{U_{C}}, \quad U_{C}=S \sum u^{k} \mathfrak{t}_{k}-S^{2} \log a
$$

Now one realizes that $Z_{C}(\tilde{\mathfrak{t}})$ depends on $u$ and, therefore, $Z_{C}$ at the 1.h.s. and the r.h.s. in (8.29) can not be the same function. Indeed,

$$
\frac{\partial Z_{C}}{\partial u}=\frac{\partial U_{C}}{\partial u}+\sum_{k=1}^{\infty} \frac{\partial \tilde{\mathfrak{t}}_{k}}{\partial u} \frac{\partial Z_{C}}{\partial \tilde{\mathfrak{t}}_{k}}=a^{2} S \tilde{\mathfrak{t}}_{1}+\frac{a^{2}}{2} \sum_{k=1}^{\infty}(k+1) \tilde{\mathfrak{t}}_{k+1} \frac{\partial Z_{C}}{\partial \tilde{\mathfrak{t}}_{k}} \sim L_{-1} Z_{C}
$$

which is non-zero for the complex model. This means that, for the complex matrix model, there is only rescaling $z \rightarrow a z$, which is generated by

$$
\exp \left(\log a L_{0}\right)
$$




\section{Appendix I Explicit expansions of partition functions}

In this Appendix we manifestly list first terms of expansions for various free energies (partition function) discussed in the paper. Throughout this and the next Appendices we use only the time variables with superscript $\Sigma$, which we omit for the sake of simplicity. We hope this could not confuse the reader. The most effective way to get the expansions is to use the corresponding Virasoro constraints, which lead to a chain of relations that can be solved recurrently [1.

\section{A1.1 $\bullet Z_{G}(t)$}

Expansion for the Gaussian free energy can be extracted from [1] for the Gaussian potential $\left(T_{k}=\right.$ $\left.\delta_{k, 2} / 2\right)$ as a series in $t_{k}$ (we restrict ourselves with values of $k$ ranging from 0 to 10):

$$
\begin{gathered}
F^{(0)}=S^{2} \log S-\frac{3}{4} S^{2}+\left(S t_{0}+S^{2} t_{2}+2 S^{3} t_{4}+5 S^{4} t_{6}+14 S^{5} t_{8}\right)+ \\
+\frac{t_{1}^{2}}{2} S+\left(t_{2}^{2}+3 t_{1} t_{3}\right) S^{2}+\left(6 t_{3}^{2}+10 t_{1} t_{5}+8 t_{2} t_{4}\right) S^{3}+\left(18 t_{4}^{2}+35 t_{1} t_{7}+30 t_{2} t_{6}+45 t_{3} t_{5}\right) S^{4}+ \\
+\left(90 t_{5}^{2}+126 t_{1} t_{9}+112 t_{2} t_{8}+168 t_{3} t_{7}+144 t_{4} t_{6}\right) S^{5}+O\left(S^{6}\right)+O\left(t^{3}\right) \\
F^{(1)}=-\frac{1}{12} \log S+\left(t_{4} S+10 t_{6} S^{2}+70 t_{8} S^{3}\right)+ \\
+\left(\frac{3}{2} t_{3}^{2}+5 t_{1} t_{5}+4 t_{2} t_{4}\right) S+\left(30 t_{4}^{2}+70 t_{1} t_{7}+60 t_{2} t_{6}+60 t_{3} t_{5}\right) S^{2}+ \\
+\left(300 t_{5}^{2}+630 t_{1} t_{9}+560 t_{2} t_{8}+630 t_{3} t_{7}+600 t_{4} t_{6}\right) S^{3}+O\left(S^{4}\right)+O\left(t^{3}\right) \\
F^{(2)}=-\frac{1}{240 S^{2}}+\left(21 S t_{8}+483 S^{2} t_{10}\right)+ \\
\left(178 t_{8} t_{2}+156 t_{4} t_{6}+\frac{165}{2} t_{5}{ }^{2}+189 t_{1} t_{9}+147 t_{3} t_{7}\right) S^{1} \\
+\left(4760 t_{8} t_{4}+2385 t_{6}^{2}+4725 t_{3} t_{9}+4830 t_{2} t_{10}+4795 t_{5} t_{7}\right) S^{2} \\
+\left(80640 t_{10} t_{4}+80640 t_{5} t_{9}+80640 t_{8} t_{6}+40670 t_{7}^{2}\right) S^{3}+O\left(S^{4}\right)+O\left(t^{3}\right)
\end{gathered}
$$

The terms not depending on times in these expressions come from the normalization volume of the unitary group in (2.11) that can be calculated using the Stirling formula in (2.12), see [1]. ${ }^{16}$

\section{A1.2 $\bullet Z_{K}(t)$}

Explicit expansion of the function $Z_{K}(t)$ can be obtained in the simplest way also from the Virasoro constraints, (2.13) by iterations [1]. Inserting into these constraints $Z_{K}(t)$ as a series in $g^{2},{ }^{17}$

$$
\log Z_{K}(\tau)=\sum_{h=0}^{\infty} \mathcal{F}_{K}^{(p)}(\tau)\left(8 g^{2}\right)^{p-1}
$$

\footnotetext{
${ }^{16}[2.12$ is also in charge of some correcting constant terms that are not consistent with the genus expansion 1 .

${ }^{17}$ Note that this expansion differs from (2.33) by a trivial factor.
} 
One obtains

$$
\begin{aligned}
& \mathcal{F}_{K}^{(0)}(\tau)=1 / 6 \tau_{0}^{3}+ \\
& +1 / 4 \tau_{0}{ }^{3} \tau_{1}+\frac{5}{32} \tau_{0}{ }^{4} \tau_{2}+ \\
& 3 / 8 \tau_{0}{ }^{3} \tau_{1}{ }^{2}+\frac{45}{64} \tau_{0}{ }^{4} \tau_{1} \tau_{2}+\frac{7}{64} \tau_{0}{ }^{5} \tau_{3}+\frac{45}{128} \tau_{0}{ }^{5} \tau_{2}{ }^{2}+ \\
& \frac{9}{16} \tau_{0}{ }^{3} \tau_{1}{ }^{3}+\frac{135}{64} \tau_{0}{ }^{4} \tau_{1}{ }^{2} \tau_{2}+\frac{21}{32} \tau_{0}^{5} \tau_{1} \tau_{3}+\frac{675}{256} \tau_{0}{ }^{5} \tau_{1} \tau_{2}{ }^{2}+\frac{21}{256} \tau_{0}{ }^{6} \tau_{4}+\frac{175}{256} \tau_{0}{ }^{6} \tau_{2} \tau_{3}+\frac{1125}{1024} \tau_{0}{ }^{6} \tau_{2}{ }^{3}+ \\
& \frac{27}{32} \tau_{0}{ }^{3} \tau_{1}{ }^{4}+\frac{675}{128} \tau_{0}{ }^{4} \tau_{1}{ }^{3} \tau_{2}+\frac{315}{128} \tau_{0}{ }^{5} \tau_{1}{ }^{2} \tau_{3}+\frac{6075}{512} \tau_{0}{ }^{5} \tau_{1}{ }^{2} \tau_{2}{ }^{2}+\frac{315}{512} \tau_{0}{ }^{6} \tau_{1} \tau_{4}+\frac{1575}{256} \tau_{0}{ }^{6} \tau_{1} \tau_{2} \tau_{3}+ \\
& +\frac{23625}{2048} \tau_{0}{ }^{6} \tau_{1} \tau_{2}{ }^{3}+\frac{33}{512} \tau_{0}{ }^{7} \tau_{5}+\frac{175}{512} \tau_{0}{ }^{7} \tau_{3}{ }^{2}+\frac{675}{1024} \tau_{0}{ }^{7} \tau_{2} \tau_{4}+\frac{7875}{2048} \tau_{0}{ }^{7} \tau_{2}{ }^{2} \tau_{3}+\frac{16875}{4096} \tau_{0}{ }^{7} \tau_{2}{ }^{4}+ \\
& \frac{81}{64} \tau_{0}{ }^{3} \tau_{1}{ }^{5}+\frac{6075}{512} \tau_{0}{ }^{4} \tau_{1}{ }^{4} \tau_{2}+\frac{945}{128} \tau_{0}{ }^{5} \tau_{1}{ }^{3} \tau_{3}+\frac{42525}{1024} \tau_{0}{ }^{5} \tau_{1}{ }^{3} \tau_{2}{ }^{2}+\frac{2835}{1024} \tau_{0}{ }^{6} \tau_{1}{ }^{2} \tau_{4}+\frac{33075}{1024} \tau_{0}{ }^{6} \tau_{1}{ }^{2} \tau_{2} \tau_{3}+ \\
& +\frac{70875}{1024} \tau_{0}{ }^{6} \tau_{1}{ }^{2} \tau_{2}{ }^{3}+\frac{297}{512} \tau_{0}{ }^{7} \tau_{1} \tau_{5}+\frac{3675}{1024} \tau_{0}{ }^{7} \tau_{1} \tau_{3}{ }^{2}+\frac{14175}{2048} \tau_{0}{ }^{7} \tau_{1} \tau_{2} \tau_{4}+\frac{23625}{512} \tau_{0}{ }^{7} \tau_{1} \tau_{2}{ }^{2} \tau_{3}+ \\
& +\frac{455625}{8192} \tau_{0}{ }^{7} \tau_{1} \tau_{2}{ }^{4}+\frac{429}{8192} \tau_{0}^{8} \tau_{6}+\frac{11025}{16384} \tau_{0}{ }^{8} \tau_{3} \tau_{4}+\frac{10395}{16384} \tau_{0}{ }^{8} \tau_{2} \tau_{5}+\frac{18375}{4096} \tau_{0}{ }^{8} \tau_{2} \tau_{3}{ }^{2}+ \\
& +\frac{70875}{16384} \tau_{0}{ }^{8} \tau_{2}{ }^{2} \tau_{4}+\frac{354375}{16384} \tau_{0}{ }^{8} \tau_{2}{ }^{3} \tau_{3}+\frac{2278125}{131072} \tau_{0}{ }^{8} \tau_{2}{ }^{5}+\ldots \\
& \mathcal{F}_{K}^{(1)}(\tau)=1 / 16 \tau_{1}+\frac{5}{32} \tau_{0} \tau_{2}+ \\
& \frac{3}{64} \tau_{1}^{2}+\frac{15}{32} \tau_{0} \tau_{1} \tau_{2}+\frac{35}{128} \tau_{0}^{2} \tau_{3}+\frac{75}{128} \tau_{0}^{2} \tau_{2}{ }^{2}+ \\
& \frac{3}{64} \tau_{1}^{3}+\frac{135}{128} \tau_{0} \tau_{1}^{2} \tau_{2}+\frac{315}{256} \tau_{0}^{2} \tau_{1} \tau_{3}+\frac{225}{64} \tau_{0}^{2} \tau_{1} \tau_{2}{ }^{2}+\frac{105}{256} \tau_{0}{ }^{3} \tau_{4}+\frac{1225}{512} \tau_{0}^{3} \tau_{2} \tau_{3}+\frac{375}{128} \tau_{0}{ }^{3} \tau_{2}{ }^{3}+ \\
& \frac{27}{512} \tau_{1}{ }^{4}+\frac{135}{64} \tau_{0} \tau_{1}{ }^{3} \tau_{2}+\frac{945}{256} \tau_{0}^{2} \tau_{1}{ }^{2} \tau_{3}+\frac{3375}{256} \tau_{0}{ }^{2} \tau_{1}{ }^{2} \tau_{2}{ }^{2}+\frac{315}{128} \tau_{0}{ }^{3} \tau_{1} \tau_{4}+\frac{18375}{1024} \tau_{0}{ }^{3} \tau_{1} \tau_{2} \tau_{3}+ \\
& +\frac{3375}{128} \tau_{0}{ }^{3} \tau_{1} \tau_{2}{ }^{3}+\frac{1155}{2048} \tau_{0}{ }^{4} \tau_{5}+\frac{8575}{4096} \tau_{0}{ }^{4} \tau_{3}{ }^{2}+\frac{17325}{4096} \tau_{0}{ }^{4} \tau_{2} \tau_{4}+\frac{154875}{8192} \tau_{0}{ }^{4} \tau_{2}{ }^{2} \tau_{3}+\frac{16875}{1024} \tau_{0}{ }^{4} \tau_{2}{ }^{4}+ \\
& \frac{81}{1280} \tau_{1}^{5}+\frac{2025}{512} \tau_{0} \tau_{1}{ }^{4} \tau_{2}+\frac{4725}{512} \tau_{0}^{2} \tau_{1}{ }^{3} \tau_{3}+\frac{10125}{256} \tau_{0}{ }^{2} \tau_{1}{ }^{3} \tau_{2}{ }^{2}+\frac{4725}{512} \tau_{0}{ }^{3} \tau_{1}{ }^{2} \tau_{4}+\frac{165375}{2048} \tau_{0}{ }^{3} \tau_{1}{ }^{2} \tau_{2} \tau_{3}+ \\
& +\frac{70875}{512} \tau_{0}{ }^{3} \tau_{1}{ }^{2} \tau_{2}{ }^{3}+\frac{17325}{4096} \tau_{0}{ }^{4} \tau_{1} \tau_{5}+\frac{77175}{4096} \tau_{0}{ }^{4} \tau_{1} \tau_{3}{ }^{2}+\frac{155925}{4096} \tau_{0}{ }^{4} \tau_{1} \tau_{2} \tau_{4}+\frac{3252375}{16384} \tau_{0}{ }^{4} \tau_{1} \tau_{2}{ }^{2} \tau_{3}+ \\
& +\frac{50625}{256} \tau_{0}{ }^{4} \tau_{1} \tau_{2}{ }^{4}+\frac{3003}{4096} \tau_{0}^{5} \tau_{6}+\frac{55125}{8192} \tau_{0}{ }^{5} \tau_{3} \tau_{4}+\frac{3465}{512} \tau_{0}{ }^{5} \tau_{2} \tau_{5}+\frac{18375}{512} \tau_{0}{ }^{5} \tau_{2} \tau_{3}{ }^{2}+ \\
& +\frac{590625}{16384} \tau_{0}{ }^{5} \tau_{2}{ }^{2} \tau_{4}+\frac{4764375}{32768} \tau_{0}{ }^{5} \tau_{2}{ }^{3} \tau_{3}+\frac{50625}{512} \tau_{0}{ }^{5} \tau_{2}{ }^{5}+\ldots
\end{aligned}
$$




$$
\begin{aligned}
& \mathcal{F}_{K}^{(2)}(\tau)=\frac{105}{2048} \tau_{4}+\frac{1015}{4096} \tau_{2} \tau_{3}+\frac{525}{2048} \tau_{2}^{3}+ \\
& +\frac{945}{4096} \tau_{1} \tau_{4}+\frac{3045}{2048} \tau_{1} \tau_{2} \tau_{3}+\frac{7875}{4096} \tau_{1} \tau_{2}^{3}+\frac{1155}{4096} \tau_{0} \tau_{5}+\frac{7105}{8192} \tau_{0} \tau_{3}^{2}+\frac{3465}{2048} \tau_{0} \tau_{2} \tau_{4}+ \\
& +\frac{13125}{2048} \tau_{0} \tau_{2}^{2} \tau_{3}+\frac{39375}{8192} \tau_{0} \tau_{2}{ }^{4}+ \\
& +\frac{2835}{4096} \tau_{1}^{2} \tau_{4}+\frac{45675}{8192} \tau_{1}^{2} \tau_{2} \tau_{3}+\frac{70875}{8192} \tau_{1}^{2} \tau_{2}^{3}+\frac{3465}{2048} \tau_{0} \tau_{1} \tau_{5}+\frac{106575}{16384} \tau_{0} \tau_{1} \tau_{3}^{2}+\frac{51975}{4096} \tau_{0} \tau_{1} \tau_{2} \tau_{4}+ \\
& +\frac{118125}{2048} \tau_{0} \tau_{1} \tau_{2}^{2} \tau_{3}+\frac{826875}{16384} \tau_{0} \tau_{1} \tau_{2}{ }^{4}+\frac{15015}{16384} \tau_{0}^{2} \tau_{6}+\frac{112455}{16384} \tau_{0}^{2} \tau_{3} \tau_{4}+\frac{3465}{512} \tau_{0}{ }^{2} \tau_{2} \tau_{5}+ \\
& +\frac{2002875}{65536} \tau_{0}^{2} \tau_{2} \tau_{3}{ }^{2}+\frac{496125}{16384} \tau_{0}^{2} \tau_{2}^{2} \tau_{4}+\frac{433125}{4096} \tau_{0}^{2} \tau_{2}{ }^{3} \tau_{3}+\frac{4134375}{65536} \tau_{0}{ }^{2} \tau_{2}{ }^{5}+ \\
& \frac{4673375}{131072} \tau_{0}{ }^{3} \tau_{3}{ }^{3}+\frac{337365}{32768} \tau_{0}{ }^{3} \tau_{4}{ }^{2}+\frac{14175}{8192} \tau_{1}{ }^{3} \tau_{4}+\frac{1299375}{1024} \tau_{0}{ }^{2} \tau_{1} \tau_{2}{ }^{3} \tau_{3}+\frac{1012095}{16384} \tau_{0}{ }^{2} \tau_{1} \tau_{3} \tau_{4}+\ldots \\
& \mathcal{F}_{K}^{(3)}(\tau)=\frac{25025}{131072} \tau_{7}+\frac{191205}{262144} \tau_{4}^{2}+\frac{193655}{131072} \tau_{3} \tau_{5}+\frac{3570875}{1572864} \tau_{3}{ }^{3}+\frac{385385}{262144} \tau_{2} \tau_{6}+\frac{1765575}{131072} \tau_{2} \tau_{3} \tau_{4}+ \\
& +\frac{883575}{131072} \tau_{2}{ }^{2} \tau_{5}+\frac{18834375}{524288} \tau_{2}{ }^{2} \tau_{3}{ }^{2}+\frac{6260625}{262144} \tau_{2}{ }^{3} \tau_{4}+\frac{37996875}{524288} \tau_{2}{ }^{4} \tau_{3}+\frac{34453125}{1048576} \tau_{2}{ }^{6}+\ldots
\end{aligned}
$$

\section{$\mathrm{A} 1.3 \bullet \tilde{Z}_{K}$}

$\tilde{Z}_{K}$ is given by definition as the solution to the Virasoro constraints

$$
\begin{gathered}
\hat{\tilde{L}}_{(n)}^{K} \tilde{Z}_{K}=0 \quad n=0 \ldots \infty \\
\hat{\tilde{L}}_{(n)}^{K}=\sum_{k>0}\left(k+\frac{1}{2}\right)\left(\mathcal{T}_{k}-c \delta_{k, 0}\right) \frac{\partial}{\partial \mathcal{T}_{k+n}}+g^{2} \sum_{a+b=n-1} \frac{\partial^{2}}{\partial \mathcal{T}_{a} \partial \mathcal{T}_{b}}+\frac{\delta_{n, 0}}{16}
\end{gathered}
$$

$\tilde{Z}_{K}$ possesses the very simple genus expansion, that is,

$$
\begin{gathered}
\tilde{\mathcal{F}}^{(0)}=0 \\
\tilde{\mathcal{F}}^{(1)}=-\frac{1}{8} \log \left(c-\mathcal{T}_{0}\right) \\
\tilde{\mathcal{F}}^{(2)}=-\frac{9 \mathcal{T}_{1}}{32\left(\mathcal{T}_{0}-c\right)^{3}}
\end{gathered}
$$

\section{$\mathbf{A 1 . 4} \bullet Z_{C}(\mathfrak{t})$}

In order to get the expansion of the partition function of the complex matrix model, one can again use the Virasoro constraints, (2.17). Similarly to the case Gaussian model [1, they can be recast in the form of loop equations which being expanded in the $g$-series lead to recurrent relations for the resolvents (the details can be found in [1])

$$
\begin{gathered}
\rho_{C}^{(k \mid m)}\left(z_{1}, \ldots, z_{m}\right) \equiv \sum_{n_{1}, \ldots, n_{m}} \frac{1}{z_{1}^{n_{1}+1} \ldots z_{m}^{n_{m}+1}} \frac{\partial^{n_{1}+\ldots+n_{m}} F_{C}^{(k)}}{\partial \mathfrak{t}_{n_{1}} \ldots \partial \mathfrak{t}_{n_{m}}} \\
\log Z_{C}\left(\mathfrak{t} \mid g^{2}\right)=\sum_{h=0}^{\infty} g^{2 h-2} F_{C}^{(h)}
\end{gathered}
$$


which, for a generic polynomial potential $W(z)$ looks like

$$
\begin{gathered}
\frac{W_{C}^{\prime}(z) \rho_{W_{C}}^{(p \mid m+1)}\left(z, z_{1}, \ldots, z_{m}\right)}{2}-f_{W_{C}}^{(p \mid m+1)}\left(z \mid z_{1}, \ldots, z_{m}\right)= \\
=\sum_{q} \sum_{m_{1}+m_{2}=m} \rho_{W_{C}}^{\left(q \mid m_{1}+1\right)}\left(z, z_{i_{1}}, \ldots, z_{i_{m_{1}}}\right) \rho_{W_{C}}^{\left(p-q \mid m_{2}+1\right)}\left(z, z_{j_{1}}, \ldots, z_{j_{m_{2}}}\right)+ \\
+\sum_{i=1}^{m} \frac{\partial}{\partial z_{i}} \frac{z \rho_{W_{C}}^{(p \mid m)}\left(z, z_{1}, \ldots, \check{z}_{i}, \ldots, z_{m}\right)-z_{1} \rho_{W}^{(p \mid m)}\left(z_{1}, \ldots, z_{m}\right)}{2\left(z^{2}-z_{i}^{2}\right)}+\hat{\nabla}(z) \rho_{W_{C}}^{(p-1 \mid m+1)}\left(z, z_{1}, \ldots, z_{m}\right) . \\
\rho_{C}^{(0 \mid 1)}(z)=\frac{\frac{W^{\prime}(z)}{2}-y_{C}(z)}{2} \\
y_{C}(z)^{2}=\frac{W^{\prime 2}}{4}-4 \check{R}(z) F_{C}^{(0)}
\end{gathered}
$$

where $\check{R}(z)$ is the check operator introduced in [13], etc. For the Gaussian complex model with $W=z^{2}$

$$
\begin{gathered}
\rho_{C}^{(0 \mid 1)}(z)=\frac{z-\sqrt{z^{2}-4 S}}{2} \\
y\left(z_{1}\right) \rho_{C}^{(0 \mid 2)}\left(z_{1}, z_{2}\right)=\partial_{z_{2}} \frac{z_{1} \rho_{C}^{(0 \mid 1)}\left(z_{1}\right)-z_{2} \rho_{C}^{(0 \mid 1)}\left(z_{2}\right)}{2\left(z_{1}^{2}-z_{2}^{2}\right)} \\
\rho_{C}^{(0 \mid 2)}\left(z_{1}, z_{2}\right)=\frac{1}{2\left(z_{1}^{2}-z_{2}^{2}\right)^{2}}\left(\frac{z_{1}^{2} z_{2}^{2}-2 S\left(z_{1}^{2}+z_{2}^{2}\right)}{y_{1} y_{2}}-z_{1} z_{2}\right)
\end{gathered}
$$

(see, for example [48])

$$
\begin{gathered}
y\left(z_{1}\right) \rho_{C}^{(1 \mid 1)}\left(z_{1}\right)=\rho_{C}^{(0 \mid 2)}\left(z_{1}, z_{1}\right) \\
\rho_{C}^{(1 \mid 1)}(z)=\frac{S^{2}}{z^{2} y(z)^{5}} \\
y\left(z_{1}\right) \rho_{C}^{(0 \mid 3)}\left(z_{1}, z_{2}, z_{3}\right)=2 \rho_{C}^{(0 \mid 2)}\left(z_{1}, z_{2}\right) \rho_{C}^{(0 \mid 2)}\left(z_{1}, z_{3}\right)+ \\
+\partial_{z_{2}} \frac{z_{1} \rho_{C}^{(0 \mid 2)}\left(z_{1}, z_{3}\right)-z_{2} \rho_{C}^{(0 \mid 2)}\left(z_{2}, z_{3}\right)}{2\left(z_{1}^{2}-z_{2}^{2}\right)}+\partial_{z_{3}} \frac{z_{1} \rho_{C}^{(0 \mid 2)}\left(z_{1}, z_{2}\right)-z_{3} \rho_{C}^{(0 \mid 2)}\left(z_{2}, z_{3}\right)}{2\left(z_{1}^{2}-z_{3}^{2}\right)} \\
\rho_{C}^{(0 \mid 3)}\left(z_{1}, z_{2}, z_{3}\right)=2 \frac{S^{2}}{\left(z_{1}^{2}-4 S\right)^{3 / 2}\left(z_{3}^{2}-4\right)^{3 / 2}\left(z_{2}^{2}-4 S\right)^{3 / 2}} \\
y\left(z_{1}\right) \rho_{C}^{(1 \mid 2)}\left(z_{1}, z_{2}\right)=2 \rho_{C}^{(0 \mid 2)}\left(z_{1}, z_{2}\right) \rho_{C}^{(1 \mid 1)}\left(z_{1}\right)+\rho_{C}^{(0 \mid 3)}\left(z_{1}, z_{1}, z_{2}\right)+\partial_{z_{2}} \frac{z_{1} \rho_{C}^{(1 \mid 1)}\left(z_{1}\right)-z_{2} \rho_{C}^{(1 \mid 1)}\left(z_{2}\right)}{2\left(z_{1}^{2}-z_{2}^{2}\right)} \\
\rho_{C}^{(1 \mid 2)}\left(z_{1}, z_{2}\right)=\frac{S^{2}}{z_{1}^{2} z_{2}^{2} y_{1}^{7} y_{2}^{7}}\left(3 z_{2}^{2} z_{1}^{6}+3 z_{2}^{6} z_{1}^{2}+2 z_{2}^{4} z_{1}^{4}\right. \\
\left.-2\left(z_{1}^{6}+17 z_{1}^{4} z_{2}^{2}+17 z_{1}^{2} z_{2}^{4}+z_{2}^{6}\right) S+8\left(3 z_{1}^{4}+19 z_{1}^{2} z_{2}^{2}+3 z_{2}^{4}\right) S^{2}-96\left(z_{1}^{2}+z_{2}^{2}\right) S^{3}+128 S^{4}\right) \\
y\left(z_{1}\right) \rho_{C}^{(2 \mid 1)}\left(z_{1}\right)=\left(\rho_{C}^{(1 \mid 1)}\left(z_{1}\right)\right)^{2}+\rho_{C}^{(1 \mid 2)}\left(z_{1}, z_{1}\right) \\
\rho_{C}^{(2 \mid 1)}(z)=\frac{\left(9 S^{2}-8 z^{2} S+8 z^{4}\right) S^{2}}{z^{4} y(z)^{11}}
\end{gathered}
$$




$$
\begin{aligned}
& F_{C}^{(0)}=\mathfrak{t}_{0} S+S^{2} \mathfrak{t}_{1}+2 S^{3} \mathfrak{t}_{2}+5 S^{4} \mathfrak{t}_{3}+14 S^{5} \mathfrak{t}_{4}+42 S^{6} \mathfrak{t}_{5}+132 S^{7} \mathfrak{t}_{6}+\ldots+ \\
& \frac{1}{2} \mathfrak{t}_{1}{ }^{2} S^{2}+4 \mathfrak{t}_{1} \mathfrak{t}_{2} S^{3}+\left(15 \mathfrak{t}_{3} \mathfrak{t}_{1}+9 \mathfrak{t}_{2}{ }^{2}\right) S^{4}+\left(56 \mathfrak{t}_{4} \mathfrak{t}_{1}+72 \mathfrak{t}_{3} \mathfrak{t}_{2}\right) S^{5}+\left(150 \mathfrak{t}_{3}{ }^{2}+210 \mathfrak{t}_{5} \mathfrak{t}_{1}+280 \mathfrak{t}_{4} \mathfrak{t}_{2}\right) S^{6}+ \\
& +\left(1080 \mathfrak{t}_{5} \mathfrak{t}_{2}+1200 \mathfrak{t}_{4} \mathfrak{t}_{3}+792 \mathfrak{t}_{6} \mathfrak{t}_{1}\right) S^{7}+\left(4725 \mathfrak{t}_{3} \mathfrak{t}_{5}+4158 \mathfrak{t}_{2} \mathfrak{t}_{6}+2450 \mathfrak{t}_{4}^{2}\right) S^{8}+ \\
& +\left(18480 \mathfrak{t}_{3} \mathfrak{t}_{6}+19600 \mathfrak{t}_{4} \mathfrak{t}_{5}\right) S^{9}+\left(39690 \mathfrak{t}_{5}{ }^{2}+77616 \mathfrak{t}_{4} \mathfrak{t}_{6}\right) S^{10}+317520 \mathfrak{t}_{5} \mathfrak{t}_{6} S^{11}+640332 \mathfrak{t}_{6}{ }^{2} S^{12}+\ldots+ \\
& +\frac{1}{3} \mathfrak{t}_{1}{ }^{3} S^{2}+6 \mathfrak{t}_{2} \mathfrak{t}_{1}{ }^{2} S^{3}+\left(30 \mathfrak{t}_{3} \mathfrak{t}_{1}{ }^{2}+36 \mathfrak{t}_{2}{ }^{2} \mathfrak{t}_{1}\right) S^{4}+\left(140 \mathfrak{t}_{4} \mathfrak{t}_{1}{ }^{2}+360 \mathfrak{t}_{3} \mathfrak{t}_{2} \mathfrak{t}_{1}+72 \mathfrak{t}_{2}{ }^{3}\right) S^{5}+ \\
& +\left(900 \mathfrak{t}_{1} \mathfrak{t}_{3}{ }^{2}+630 \mathfrak{t}_{5} \mathfrak{t}_{1}{ }^{2}+1080 \mathfrak{t}_{3} \mathfrak{t}_{2}{ }^{2}+1680 \mathfrak{t}_{4} \mathfrak{t}_{2} \mathfrak{t}_{1}\right) S^{6}+ \\
& +\left(8400 \mathfrak{t}_{4} \mathfrak{t}_{3} \mathfrak{t}_{1}+5400 \mathfrak{t}_{2} \mathfrak{t}_{3}^{2}+7560 \mathfrak{t}_{5} \mathfrak{t}_{2} \mathfrak{t}_{1}+5040 \mathfrak{t}_{4} \mathfrak{t}_{2}{ }^{2}\right) S^{7}+ \\
& +\left(22680 \mathfrak{t}_{5} \mathfrak{t}_{2}{ }^{2}+50400 \mathfrak{t}_{4} \mathfrak{t}_{2} \mathfrak{t}_{3}+19600 \mathfrak{t}_{4}{ }^{2} \mathfrak{t}_{1}+9000 \mathfrak{t}_{3}{ }^{3}+37800 \mathfrak{t}_{5} \mathfrak{t}_{3} \mathfrak{t}_{1}\right) S^{8}+ \\
& +\left(176400 \mathfrak{t}_{4} \mathfrak{t}_{5} \mathfrak{t}_{1}+126000 \mathfrak{t}_{4} \mathfrak{t}_{3}{ }^{2}+226800 \mathfrak{t}_{5} \mathfrak{t}_{3} \mathfrak{t}_{2}+117600 \mathfrak{t}_{2} \mathfrak{t}_{4}{ }^{2}\right) S^{9}+ \\
& +\left(1058400 \mathfrak{t}_{4} \mathfrak{t}_{2} \mathfrak{t}_{5}+588000 \mathfrak{t}_{4}^{2} \mathfrak{t}_{3}+396900 \mathfrak{t}_{5}{ }^{2} \mathfrak{t}_{1}+567000 \mathfrak{t}_{5} \mathfrak{t}_{3}^{2}\right) S^{10}+ \\
& +\left(\frac{2744000}{3} \mathfrak{t}_{4}{ }^{3}+2381400 \mathfrak{t}_{5}{ }^{2} \mathfrak{t}_{2}+5292000 \mathfrak{t}_{4} \mathfrak{t}_{5} \mathfrak{t}_{3}\right) S^{11}+\left(11907000 \mathfrak{t}_{3} \mathfrak{t}_{5}{ }^{2}+12348000 \mathfrak{t}_{4}{ }^{2} \mathfrak{t}_{5}\right) S^{12}+ \\
& +55566000 \mathfrak{t}_{4} \mathfrak{t}_{5}{ }^{2} S^{13}+83349000 \mathfrak{t}_{5}{ }^{3} S^{14}+\ldots \\
& F_{C}^{(1)}=\mathfrak{t}_{3} S^{2}+10 \mathfrak{t}_{4} S^{3}+70 S^{4} \mathfrak{t}_{5}+420 S^{5} \mathfrak{t}_{6}+2310 S^{6} \mathfrak{t}_{7}+12012 S^{7} \mathfrak{t}_{8}+60060 S^{8} \mathfrak{t}_{9}+\ldots+ \\
& +\left(3 \mathfrak{t}_{1} \mathfrak{t}_{3}+\mathfrak{t}_{2}^{2}\right) S^{2}+\left(36 \mathfrak{t}_{2} \mathfrak{t}_{3}+40 \mathfrak{t}_{1} \mathfrak{t}_{4}\right) S^{3}+\left(174 \mathfrak{t}_{3}^{2}+350 \mathfrak{t}_{1} \mathfrak{t}_{5}+360 \mathfrak{t}_{2} \mathfrak{t}_{4}\right) S^{4}+ \\
& +\left(2800 \mathfrak{t}_{2} \mathfrak{t}_{5}+2520 \mathfrak{t}_{1} \mathfrak{t}_{6}+2760 \mathfrak{t}_{4} \mathfrak{t}_{3}\right) S^{5}+\left(18900 \mathfrak{t}_{6} \mathfrak{t}_{2}+19110 \mathfrak{t}_{5} \mathfrak{t}_{3}+9500 \mathfrak{t}_{4}^{2}+16170 \mathfrak{t}_{1} \mathfrak{t}_{7}\right) S^{6}+ \\
& +\left(96096 \mathfrak{t}_{8} \mathfrak{t}_{1}+120400 \mathfrak{t}_{5} \mathfrak{t}_{4}+116424 \mathfrak{t}_{7} \mathfrak{t}_{2}+120456 \mathfrak{t}_{6} \mathfrak{t}_{3}\right) S^{7}+ \\
& +\left(357700 \mathfrak{t}_{5}^{2}+709632 \mathfrak{t}_{7} \mathfrak{t}_{3}+715680 \mathfrak{t}_{6} \mathfrak{t}_{4}+672672 \mathfrak{t}_{8} \mathfrak{t}_{2}\right) S^{8}+ \\
& +\left(4057200 \mathfrak{t}_{6} \mathfrak{t}_{5}+4047120 \mathfrak{t}_{7} \mathfrak{t}_{4}+3974256 \mathfrak{t}_{8} \mathfrak{t}_{3}\right) S^{9}+\left(21999120 \mathfrak{t}_{8} \mathfrak{t}_{4}+11086740 \mathfrak{t}_{6}{ }^{2}+22152900 \mathfrak{t}_{7} \mathfrak{t}_{5}\right) S^{10}+ \\
& +\left(117237120 \mathfrak{t}_{8} \mathfrak{t}_{5}+117588240 \mathfrak{t}_{7} \mathfrak{t}_{6}\right) S^{11}+\left(304371144 \mathfrak{t}_{7}^{2}+607999392 \mathfrak{t}_{8} \mathfrak{t}_{6}\right) S^{12}+ \\
& +3085546464 \mathfrak{t}_{8} \mathfrak{t}_{7} S^{13}+7688496816 \mathfrak{t}_{8}{ }^{2} S^{14}+\ldots \\
& F_{C}^{(2)}=8 \mathfrak{t}_{5} S^{2}+168 \mathfrak{t}_{6} S^{3}+2121 S^{4} \mathfrak{t}_{7}+20790 S^{5} \mathfrak{t}_{8}+174174 S^{6} \mathfrak{t}_{9}+ \\
& +1309308 \mathfrak{t}_{10} S^{7}+9087078 S^{8} \mathfrak{t}_{11}+59306676 \mathfrak{t}_{12} S^{9}+\ldots
\end{aligned}
$$

\section{A1.5 $\bullet Z_{G}\left(t^{ \pm}\right)$}

In order to obtain the Gaussian free energy in terms of the ACKM moments ${ }^{18}$, one suffices to use manifest expressions $F^{(p)}$ in terms of the standard times (see the beginning of this Appendix) and insert there manifest expressions for the times through moment variables. E.g., one can use explicit formula (A1.3) for the genus two free energy (which we actually need below to check explicitly the corresponding decomposition formula, which we check starting from the genus 2 free energy only) to

\footnotetext{
${ }^{18}$ In 42 the notations used are $t_{k}^{+}=J_{k}$ and $t_{k}^{-}=M_{k}$.
} 
obtain the well-known answer

$$
\begin{gathered}
F^{(2)}=\frac{1}{256}\left(-\frac{1448}{15} \frac{1}{t_{1}^{-2} d}-80 \frac{1}{t_{1}^{+} d t_{1}^{-}}-\frac{1448}{15} \frac{1}{t_{1}^{+2} d}\right) d^{-3}-\frac{35}{384} \frac{t_{4}^{-}}{d t_{1}^{-3}}+\frac{43}{192} \frac{t_{3}^{-}}{d^{2} t_{1}^{-3}} \\
+\frac{1}{256}\left(-\frac{1448}{15} t_{1}^{-3}-12 \frac{1}{t_{1}^{+} t_{1}^{-2}}\right) t_{2}^{-} d^{-3}+\frac{35}{384} \frac{t_{4}^{+}}{d t_{1}^{+3}}+\frac{43}{192} \frac{g^{2} t_{3}^{+}}{d^{2} t_{1}^{+3}} \\
+\frac{1}{256}\left(\frac{1448}{15} t_{1}^{+-3}+12 \frac{1}{t_{1}^{+2} t_{1}^{-}}\right) t_{2}^{+} d^{-3}+\frac{29}{128} \frac{t_{2}^{-} t_{3}^{-}}{d t_{1}^{-4}}-\frac{11}{40} \frac{t_{2}^{-2}}{d^{2} t_{1}^{-4}}+\frac{1}{64} \frac{t_{2}^{+} t_{2}^{-}}{d^{2} t_{1}^{+2} t_{1}^{-2}} \\
-\frac{29}{128} \frac{t_{2}^{+} t_{3}^{+}}{d t_{1}^{+4}}-\frac{11}{40} \frac{t_{2}^{+2}}{d^{2} t_{1}^{+4}}-\frac{21}{160} \frac{g^{2} t_{2}^{-3}}{d t_{1}^{-5}}+\frac{21}{160} \frac{t_{2}^{+3}}{d t_{1}^{+5}}
\end{gathered}
$$

Indeed, one can easily check this is correct by solving (7.15) and (7.16) perturbatively in $t$ and $S$ and then substituting them into (A1.3):

$$
\begin{gathered}
A_{+}=2 \sqrt{S}+t_{1}+2 t_{2} t_{1}+2 t_{2} \sqrt{S}+\left(3 t_{2}^{2}+6 t_{1} t_{3}\right) \sqrt{S}+6 t_{3} S+\left(24 t_{1} t_{4}+24 t_{2} t_{3}\right) S \\
+12 t_{4} S^{3 / 2}+\left(60 t_{1} t_{5}+60 t_{2} t_{4}+36 t_{3}^{2}\right) S^{3 / 2}+30 t_{5} S^{2}+\left(216 t_{4} t_{3}+180 t_{5} t_{2}+180 t_{1} t_{6}\right) S^{2} \\
+60 t_{6} S^{5 / 2}+\left(420 t_{2} t_{6}+540 t_{3} t_{5}+252 t_{4}{ }^{2}+420 t_{1} t_{7}\right) S^{5 / 2}+140 t_{7} S^{3} \\
+\left(1440 t_{3} t_{6}+1120 t_{7} t_{2}+1440 t_{5} t_{4}+1120 t_{1} t_{8}\right) S^{3} \\
+280 t_{8} S^{7 / 2}+\left(3360 t_{3} t_{7}+2520 t_{8} t_{2}+3240 t_{4} t_{6}+1800 t_{5}{ }^{2}+2520 t_{1} t_{9}\right) S^{7 / 2}+630 t_{9} S^{4} \\
+\left(8400 t_{7} t_{4}+6300 t_{1} t_{10}+8400 t_{3} t_{8}+6300 t_{9} t_{2}+9000 t_{5} t_{6}\right) S^{4}+1260 t_{10} S^{9 / 2} \\
+\left(18900 t_{3} t_{9}+13860 t_{2} t_{10}+9900 t_{6}{ }^{2}+21000 t_{5} t_{7}+18480 t_{8} t_{4}\right) S^{9 / 2} \\
+\left(45360 t_{3} t_{10}+50400 t_{5} t_{8}+50400 t_{7} t_{6}+45360 t_{9} t_{4}\right) S^{5}+O\left(S^{11 / 2}\right) \\
A_{-}=-2 \sqrt{S}+t_{1}+2 t_{2} t_{1}-2 t_{2} \sqrt{S}+\left(-3 t_{2}^{2}-6 t_{1} t_{3}\right) \sqrt{S}+6 t_{3} S+\left(24 t_{1} t_{4}+24 t_{2} t_{3}\right) S \\
-12 t_{4} S^{3 / 2}+\left(-60 t_{2} t_{4}-60 t_{1} t_{5}-36 t_{3}^{2}\right) S^{3 / 2}+30 t_{5} S^{2}+\left(216 t_{4} t_{3}+180 t_{5} t_{2}+180 t_{1} t_{6}\right) S^{2} \\
-60 t_{6} S^{5 / 2}+\left(-420 t_{1} t_{7}-252 t_{4}^{2}-540 t_{3} t_{5}-420 t_{2} t_{6}\right) S^{5 / 2}+140 t_{7} S^{3} \\
+\left(1440 t_{3} t_{6}+1120 t_{7} t_{2}+1440 t_{5} t_{4}+1120 t_{1} t_{8}\right) S^{3} \\
+\left(-13860 t_{2} t_{10}-21000 t_{5} t_{7}-18480 t_{8} t_{4}-18900 t_{3} t_{9}-9900 t_{6}{ }^{2}\right) S^{9 / 2} \\
+280 t_{8} S^{7 / 2}+\left(-1800 t_{5}{ }^{2}-3360 t_{3} t_{7}-2520 t_{8} t_{2}-2520 t_{1} t_{9}-3240 t_{4} t_{6}\right) S^{7 / 2}+630 t_{9} S^{4} \\
+\left(8400 t_{7} t_{4}+6300 t_{1} t_{10}+8400 t_{3} t_{8}+6300 t_{9} t_{2}+9000 t_{5} t_{6}\right) S^{4}-1260 t_{10} S^{9 / 2} \\
+\left(4500 t_{5} t_{8}+50400 t_{7} t_{6}+45360 t_{9} t_{4}\right) S^{5} \\
+(\mathrm{A} 1.3
\end{gathered}
$$


and

$$
\begin{aligned}
& t_{1}^{-}=-1+6 t_{1} t_{3}+2 t_{2}+\left(6 t_{2} t_{3}+24 t_{1} t_{4}+6 t_{3}\right) \sqrt{S} \\
& +\left(36 t_{3}^{2}+48 t_{2} t_{4}+120 t_{1} t_{5}+24 t_{4}\right) S+\left(360 t_{1} t_{6}+60 t_{5}+180 t_{4} t_{3}+180 t_{5} t_{2}\right) S^{3 / 2} \\
& +\left(720 t_{2} t_{6}+288 t_{4}^{2}+180 t_{6}+1260 t_{1} t_{7}+900 t_{3} t_{5}\right) S^{2} \\
& +\left(1800 t_{5} t_{4}+420 t_{7}+2100 t_{7} t_{2}+2340 t_{3} t_{6}+3360 t_{1} t_{8}\right) S^{5 / 2} \\
& +\left(6720 t_{8} t_{2}+1120 t_{8}+5760 t_{4} t_{6}+8400 t_{3} t_{7}+10080 t_{1} t_{9}+3600 t_{5}^{2}\right) S^{3} \\
& +\left(15960 t_{7} t_{4}+17640 t_{9} t_{2}+2520 t_{9}+25200 t_{1} t_{10}+16200 t_{5} t_{6}+21000 t_{3} t_{8}\right) S^{7 / 2} \\
& +\left(64260 t_{3} t_{9}+6300 t_{10}+21600 t_{6}{ }^{2}+47040 t_{8} t_{4}+54600 t_{5} t_{7}+50400 t_{2} t_{10}\right) S^{4} \\
& +\left(113400 t_{7} t_{6}+126000 t_{5} t_{8}+154980 t_{10} t_{3}+120960 t_{9} t_{4}\right) S^{9 / 2} \\
& +\left(332640 t_{4} t_{10}+302400 t_{8} t_{6}+176400 t_{7}^{2}+378000 t_{9} t_{5}\right) S^{5} \\
& t_{2}^{-}=3 t_{3}+12 t_{1} t_{4}+\left(16 t_{4}+80 t_{1} t_{5}+16 t_{2} t_{4}\right) \sqrt{S} \\
& +\left(140 t_{5} t_{2}+420 t_{1} t_{6}+72 t_{4} t_{3}+70 t_{5}\right) S+\left(96 t_{4}^{2}+720 t_{2} t_{6}+240 t_{6}+1680 t_{1} t_{7}-480 t_{3} t_{5}\right) S^{3 / 2} \\
& +\left(3080 t_{7} t_{2}+1200 t_{5} t_{4}+2520 t_{3} t_{6}+770 t_{7}+6160 t_{1} t_{8}\right) S^{2} \\
& +\left(4800 t_{4} t_{6}+20160 t_{1} t_{9}+2400 t_{5}^{2}+2240 t_{8}+11200 t_{8} t_{2}+10080 t_{3} t_{7}\right) S^{5 / 2} \\
& +\left(63000 t_{1} t_{10}+16800 t_{5} t_{6}+36960 t_{3} t_{8}+20160 t_{7} t_{4}+6300 t_{9}+37800 t_{9} t_{2}\right) S^{3} \\
& +\left(61600 t_{5} t_{7}+21600 t_{6}^{2}+69440 t_{8} t_{4}+117600 t_{2} t_{10}+16800 t_{10}+120960 t_{3} t_{9}\right) S^{7 / 2} \\
& +\left(204400 t_{5} t_{8}+151200 t_{7} t_{6}+234360 t_{9} t_{4}+378000 t_{10} t_{3}\right) S^{4} \\
& +\left(436800 t_{6} t_{8}+235200 t_{7}^{2}+655200 t_{9} t_{5}+715680 t_{4} t_{10}\right) S^{9 / 2}+O\left(S^{5}\right) \\
& t_{3}^{-}=20 t_{1} t_{5}+4 t_{4}+\left(30 t_{5}+180 t_{1} t_{6}+30 t_{5} t_{2}\right) \sqrt{S} \\
& +\left(1092 t_{1} t_{7}+120 t_{3} t_{5}+312 t_{2} t_{6}+156 t_{6}\right) S+\left(5152 t_{1} t_{8}+644 t_{7}+180 t_{5} t_{4}+1080 t_{3} t_{6}+1932 t_{7} t_{2}\right) S^{3 / 2} \\
& +\left(2352 t_{8}+21168 t_{1} t_{9}+600 t_{5}^{2}+6552 t_{3} t_{7}+1872 t_{4} t_{6}+9408 t_{8} t_{2}\right) S^{2} \\
& +\left(39060 t_{9} t_{2}+6300 t_{5} t_{6}+11592 t_{7} t_{4}+30912 t_{3} t_{8}+7812 t_{9}+78120 t_{1} t_{10}\right) S^{5 / 2} \\
& +\left(24360 t_{10}+127008 t_{3} t_{9}+146160 t_{2} t_{10}+9360 t_{6}{ }^{2}+56448 t_{8} t_{4}+35560 t_{5} t_{7}\right) S^{3} \\
& +\left(234360 t_{9} t_{4}+468720 t_{10} t_{3}+83160 t_{7} t_{6}+158760 t_{8} t_{5}\right) S^{7 / 2} \\
& +\left(647640 t_{9} t_{5}+876960 t_{10} t_{4}+152880 t_{7}^{2}+325920 t_{8} t_{6}\right) S^{4}+O\left(S^{9 / 2}\right)
\end{aligned}
$$




$$
\begin{gathered}
t_{4}^{-}=30 t_{1} t_{6}+5 t_{5}+\left(336 t_{1} t_{7}+48 t_{2} t_{6}+48 t_{6}\right) \sqrt{S} \\
+\left(180 t_{3} t_{6}+2352 t_{1} t_{8}+588 t_{7} t_{2}+294 t_{7}\right) S+\left(288 t_{4} t_{6}+4224 t_{8} t_{2}+2016 t_{3} t_{7}+12672 t_{1} t_{9}+1408 t_{8}\right) S^{3 / 2} \\
+\left(900 t_{5} t_{6}+14112 t_{3} t_{8}+5814 t_{9}+23256 t_{9} t_{2}+3528 t_{7} t_{4}+58140 t_{1} t_{10}\right) S^{2} \\
+\left(10080 t_{5} t_{7}+76032 t_{3} t_{9}+25344 t_{8} t_{4}+1440 t_{6}{ }^{2}+21600 t_{10}+108000 t_{2} t_{10}\right) S^{5 / 2} \\
+\left(21840 t_{7} t_{6}+70560 t_{8} t_{5}+139536 t_{9} t_{4}+348840 t_{10} t_{3}\right) S^{3} \\
+\left(380160 t_{9} t_{5}+133440 t_{8} t_{6}+648000 t_{10} t_{4}+47040 t_{7}^{2}\right) S^{7 / 2}+O\left(S^{4}\right)
\end{gathered}
$$

Similarly,

$$
\begin{gathered}
t_{1}^{+}=-1+6 t_{1} t_{3}+2 t_{2}-\left(6 t_{2} t_{3}+24 t_{1} t_{4}+6 t_{3}\right) \sqrt{S} \\
+\left(36 t_{3}{ }^{2}+48 t_{2} t_{4}+120 t_{1} t_{5}+24 t_{4}\right) S-\left(360 t_{1} t_{6}+60 t_{5}+180 t_{4} t_{3}+180 t_{5} t_{2}\right) S^{3 / 2} \\
+\left(720 t_{2} t_{6}+288 t_{4}^{2}+180 t_{6}+1260 t_{1} t_{7}+900 t_{3} t_{5}\right) S^{2} \\
-\left(1800 t_{5} t_{4}+420 t_{7}+2100 t_{7} t_{2}+2340 t_{3} t_{6}+3360 t_{1} t_{8}\right) S^{5 / 2} \\
+\left(6720 t_{8} t_{2}+1120 t_{8}+5760 t_{4} t_{6}+8400 t_{3} t_{7}+10080 t_{1} t_{9}+3600 t_{5}{ }^{2}\right) S^{3} \\
-\left(15960 t_{7} t_{4}+17640 t_{9} t_{2}+2520 t_{9}+25200 t_{1} t_{10}+16200 t_{5} t_{6}+21000 t_{3} t_{8}\right) S^{7 / 2} \\
+\left(64260 t_{3} t_{9}+6300 t_{10}+21600 t_{6}{ }^{2}+47040 t_{8} t_{4}+54600 t_{5} t_{7}+50400 t_{2} t_{10}\right) S^{4} \\
-\left(113400 t_{7} t_{6}+126000 t_{5} t_{8}+154980 t_{10} t_{3}+120960 t_{9} t_{4}\right) S^{9 / 2} \\
+\left(332640 t_{4} t_{10}+302400 t_{8} t_{6}+176400 t_{7}^{2}+378000 t_{9} t_{5}\right) S^{5} \\
t_{2}^{+}=3 t_{3}+12 t_{1} t_{4}-\left(16 t_{4}+80 t_{1} t_{5}+16 t_{2} t_{4}\right) \sqrt{S} \\
+\left(140 t_{5} t_{2}+420 t_{1} t_{6}+72 t_{4} t_{3}+70 t_{5}\right) S-\left(96 t_{4}^{2}+720 t_{2} t_{6}+240 t_{6}+1680 t_{1} t_{7}-480 t_{3} t_{5}\right) S^{3 / 2} \\
+\left(3080 t_{7} t_{2}+1200 t_{5} t_{4}+2520 t_{3} t_{6}+770 t_{7}+6160 t_{1} t_{8}\right) S^{2} \\
+\left(436800 t_{6} t_{8}+235200 t_{7}{ }^{2}+655200 t_{9} t_{5}+715680 t_{4} t_{10}\right) S^{9 / 2}+O\left(S^{5}\right) \\
+\left(6800 t_{4} t_{6}+20160 t_{1} t_{9}+2400 t_{5}{ }^{2}+2240 t_{8}+11200 t_{8} t_{2}+10080 t_{3} t_{7}\right) S^{5 / 2} \\
+\left(61600 t_{5} t_{7}+21600 t_{6}{ }^{2}+69440 t_{8} t_{4}+117600 t_{2} t_{10}+16800 t_{10}+120960 t_{3} t_{9}\right) S^{7 / 2} \\
+\left(204400 t_{5} t_{8}+151200 t_{7} t_{6}+234360 t_{9} t_{4}+378000 t_{10} t_{3}\right) S^{4} \\
\left.+16800 t_{5} t_{6}+36960 t_{3} t_{8}+20160 t_{7} t_{4}+6300 t_{9}+37800 t_{9} t_{2}\right) S^{3} \\
+(\mathrm{A} 138)
\end{gathered}
$$




$$
\begin{gathered}
t_{3}^{+}=20 t_{1} t_{5}+4 t_{4}-\left(30 t_{5}+180 t_{1} t_{6}+30 t_{5} t_{2}\right) \sqrt{S} \\
+\left(1092 t_{1} t_{7}+120 t_{3} t_{5}+312 t_{2} t_{6}+156 t_{6}\right) S-\left(5152 t_{1} t_{8}+644 t_{7}+180 t_{5} t_{4}+1080 t_{3} t_{6}+1932 t_{7} t_{2}\right) S^{3 / 2} \\
+\left(2352 t_{8}+21168 t_{1} t_{9}+600 t_{5}^{2}+6552 t_{3} t_{7}+1872 t_{4} t_{6}+9408 t_{8} t_{2}\right) S^{2} \\
-\left(39060 t_{9} t_{2}+6300 t_{5} t_{6}+11592 t_{7} t_{4}+30912 t_{3} t_{8}+7812 t_{9}+78120 t_{1} t_{10}\right) S^{5 / 2} \\
+\left(24360 t_{10}+127008 t_{3} t_{9}+146160 t_{2} t_{10}+9360 t_{6}{ }^{2}+56448 t_{8} t_{4}+35560 t_{5} t_{7}\right) S^{3} \\
-\left(234360 t_{9} t_{4}+468720 t_{10} t_{3}+83160 t_{7} t_{6}+158760 t_{8} t_{5}\right) S^{7 / 2} \\
+\left(647640 t_{9} t_{5}+876960 t_{10} t_{4}+152880 t_{7}{ }^{2}+325920 t_{8} t_{6}\right) S^{4}+O\left(S^{9 / 2}\right) \\
t_{4}^{+}=30 t_{1} t_{6}+5 t_{5}-\left(336 t_{1} t_{7}+48 t_{2} t_{6}+48 t_{6}\right) \sqrt{S} \\
+\left(180 t_{3} t_{6}+2352 t_{1} t_{8}+588 t_{7} t_{2}+294 t_{7}\right) S-\left(288 t_{4} t_{6}+4224 t_{8} t_{2}+2016 t_{3} t_{7}+12672 t_{1} t_{9}+1408 t_{8}\right) S^{3 / 2} \\
+\left(900 t_{5} t_{6}+14112 t_{3} t_{8}+5814 t_{9}+23256 t_{9} t_{2}+3528 t_{7} t_{4}+58140 t_{1} t_{10}\right) S^{2} \\
-\left(10080 t_{5} t_{7}+76032 t_{3} t_{9}+25344 t_{8} t_{4}+1440 t_{6}{ }^{2}+21600 t_{10}+108000 t_{2} t_{10}\right) S^{5 / 2} \\
+\left(21840 t_{7} t_{6}+70560 t_{8} t_{5}+139536 t_{9} t_{4}+348840 t_{10} t_{3}\right) S^{3} \\
-\left(380160 t_{9} t_{5}+133440 t_{8} t_{6}+648000 t_{10} t_{4}+47040 t_{7}{ }^{2}\right) S^{7 / 2}+O\left(S^{4}\right)
\end{gathered}
$$

These expressions, indeed, convert (A1.30 into (A1.3). 


\section{Appendix II Explicit checks of relations between various partition functions}

\section{A2.1 Experimental check of the decomposition formula}

We check here the decomposition formula (6.11) of s.6 explicitly for the first several terms. To this end, we use the non-standard genus expansion of s.2.5.3.

For variables $t^{\prime}$ the decomposition formula acquires the form

$$
Z_{G}\left(t^{\prime} \mid S\right) e^{-U_{G}}=e^{-\hat{U}_{K}} Z_{K}\left(-\frac{g \tau^{+}}{S} \mid \frac{g^{2}}{4 S^{2}}\right) Z_{K}\left(\frac{g \tau^{-}}{S} \mid \frac{g^{2}}{4 S^{2}}\right)
$$

with

$$
\hat{U}_{G}=\frac{1}{g} \oint \rho^{(0 \mid 1)}(z) v^{\prime}(z)+\frac{1}{2 !} \oint \rho^{(0 \mid 2)}\left(z_{1}, z_{2}\right) v^{\prime}\left(z_{1}\right) v^{\prime}\left(z_{2}\right)
$$

and $U_{K}$ defined in (6.14). We check this formula in the leading order in $g$. For doing this, we need expressions for integrals of the simplest correlation functions

$$
\oint \rho^{(1 \mid 1)}(z) v^{\prime}(z) d z=\frac{1}{16 S}\left(\tau_{1}^{-}-\tau_{1}^{+}\right)-\frac{1}{24 S}\left(\tau_{0}^{-}-\tau_{0}^{+}\right)
$$

and

$$
\frac{1}{3 !} \oint \rho^{(0 \mid 3)}\left(z_{1}, z_{2}, z_{3}\right) v^{\prime}\left(z_{1}\right) v^{\prime}\left(z_{2}\right) v^{\prime}\left(z_{3}\right)=\frac{1}{2 S 3 !}\left(\left(\tau_{0}^{-}\right)^{3}-\left(\tau_{0}^{+}\right)^{3}\right)
$$

The last equality follows from

$$
\begin{gathered}
\rho^{(0 \mid 3)}\left(z_{1}, z_{2}, z_{3}\right)=\frac{2 S\left(z_{1} z_{2}+z_{1} z_{3}+z_{2} z_{3}+4 S\right)}{y\left(z_{1}\right)^{3} y\left(z_{2}\right)^{3} y\left(z_{3}\right)^{3}}= \\
=\frac{\sqrt{S}}{2 y\left(z_{1}\right) y\left(z_{2}\right) y\left(z_{3}\right)}\left(\frac{1}{\left(z_{1}-2 \sqrt{S}\right)\left(z_{2}-2 \sqrt{S}\right)\left(z_{3}-2 \sqrt{S}\right)}-\frac{1}{\left(z_{1}+2 \sqrt{S}\right)\left(z_{2}+2 \sqrt{S}\right)\left(z_{3}+2 \sqrt{S}\right)}\right)
\end{gathered}
$$

and

$$
\oint \frac{v^{\prime}(z) d z}{y(z)(z \pm 2 \sqrt{S})}=\frac{1}{\sqrt{S}} \tau_{0}^{ \pm}
$$

Substituting the explicit expansion of the Kontsevich $\tau$-function (see Appendix I)

$$
\log Z_{K}(\tau \mid \hbar)=\frac{1}{8 \hbar}\left(\frac{\tau_{0}^{3}}{3 !}+\frac{\tau_{0}^{3} \tau_{1}}{2 ! 2}+\ldots\right)+\frac{1}{16} \tau_{1}+\frac{5}{32} \tau_{0} \tau_{2}+\ldots
$$

into the decomposition formula one gets

$$
\begin{aligned}
e^{-\hat{U}_{K}} Z_{K}(- & \left.\frac{g \tau^{+}}{S} \mid \frac{g^{2}}{4 S^{2}}\right) Z_{K}\left(\frac{g \tau^{-}}{S} \mid \frac{g^{2}}{4 S^{2}}\right)=\left(1-\frac{1}{24}\left(\left(\frac{\partial}{\partial \tau_{0}^{+}}\right)^{2}+\left(\frac{\partial}{\partial \tau_{0}^{-}}\right)^{2}+\ldots\right)\right) \times \\
& \times\left(1-\frac{1}{3 !} \frac{g\left(\tau_{0}^{+}\right)^{3}}{2 S}-\frac{1}{16} \frac{g \tau_{1}^{+}}{S}+\frac{5}{32} \frac{g^{2} \tau_{0}^{+}\left(\tau_{2}^{+}+\frac{16 S}{5 ! g}\right)}{S^{2}}+\ldots\right) \times \\
& \times\left(1+\frac{1}{3 !} \frac{g\left(\tau_{0}^{-}\right)^{3}}{2 S}+\frac{1}{16} \frac{g \tau_{1}^{-}}{S}+\frac{5}{32} \frac{g^{2} \tau_{0}^{-}\left(\tau_{2}^{-}-\frac{16 S}{5 ! g}\right)}{S^{2}}+\ldots\right)= \\
=1+g & \left(\frac{1}{2 S 3 !}\left(\left(\tau_{0}^{-}\right)^{3}-\left(\tau_{0}^{+}\right)^{3}\right)+\frac{1}{16 S}\left(\tau_{1}^{-}-\tau_{1}^{+}\right)-\frac{1}{24 S}\left(\tau_{0}^{-}-\tau_{0}^{+}\right)\right)+O\left(g^{2}\right)
\end{aligned}
$$




\section{A2.2 Non-trivial dependence on $t_{0}$ and the decomposition formula}

Here we check the decomposition formula in the case of non-trivial $t_{0}$-dependence, (6.19) (see s.6.2) for the non-standard genus expansion of s.2.5.3. That is, we check that the $t_{0}$-dependence factorizes out from the formula.

Following [1, one can construct a generic branch of the partition function. In this paper, we do not study dependence on coefficients of the potential $W(z)$ and, hence, all the derivatives of $F^{(k)}$ w.r.t. these coefficients should be considered as constants determining a concrete solution.

1) For the genus 1 free energy, one considers

$$
F_{(1)}=\oint_{\infty} \rho^{(0 \mid 1)}(z) v^{\prime}(z) d z=\oint_{\infty} \rho_{G}^{(0 \mid 1)}(z) v^{\prime}(z) d z
$$

where the one-point resolvent $\rho^{(0 \mid 1)}(z)$ depends on the curve only, but not on a particular branch of the partition function chosen, and this term is canceled by $U_{G}$.

2) The genus 2 free energy is determined by the genus zero two-point function

$$
F_{(2)}\left(t^{\prime} \mid S\right)=\frac{1}{2 !} \oint \rho^{(0 \mid 2)}\left(z_{1}, z_{2}\right) v^{\prime}\left(z_{1}\right) v^{\prime}\left(z_{2}\right) d z_{1} d z_{2}=\frac{1}{2} \oint\left(\rho_{G}^{(0 \mid 2)}\left(z_{1}, z_{2}\right)-\frac{\alpha}{2 y\left(z_{1}\right) y\left(z_{2}\right)}\right) v^{\prime}\left(z_{1}\right) v^{\prime}\left(z_{2}\right) d z_{1} d z_{2}
$$

where $\alpha$ is an arbitrary constant. In terms of check-operators of [13] it can be written as

$$
\check{R} y(z)=\frac{\alpha}{y(z)}
$$

From the generic construction one concludes that, for any solution $Z$, the only dependence on $t_{0}$ comes in the combination $T_{-1}, 6$ 6.18). The operator

$$
e^{\left(t_{0}-T_{-1}\right) \frac{\partial}{\partial t_{0}}}
$$

just transform $T_{-1}$ into $t_{0}$,

$$
e^{\left(t_{0}-T_{-1}\right) \frac{\partial}{\partial t_{0}}} e^{-\hat{U}_{\infty}} \exp \left(g \mathcal{F}_{(1)}+g^{2} \mathcal{F}_{(2)}\right)=e^{\left(t_{0}-T_{-1}\right) \frac{\partial}{\partial t_{0}}} e^{\frac{-\alpha T_{-1}^{2}}{4}}=e^{-\frac{\alpha t_{0}^{2}}{4}}
$$

3) The genus 3 free energy is

$$
\begin{gathered}
F_{(3)}\left(t^{\prime} \mid S\right)=\frac{1}{3 !} \oint \rho^{(0 \mid 3)}\left(z_{1}, z_{2}, z_{3}\right) v^{\prime}\left(z_{1}\right) v^{\prime}\left(z_{2}\right) v^{\prime}\left(z_{3}\right) d z_{1} d z_{2} d z_{3}+\oint \rho^{(1 \mid 1)}(z) v^{\prime}(z) d z \\
\oint \rho^{(0 \mid 3)}\left(z_{1}, z_{2}, z_{3}\right) v^{\prime}\left(z_{1}\right) v^{\prime}\left(z_{2}\right) v^{\prime}\left(z_{3}\right) d z_{1} d z_{2} d z_{3}=\oint\left(\frac{2 S\left(z_{1} z_{2}+z_{2} z_{3}+z_{3} z_{1}+4 S\right)}{y_{G}^{3}\left(z_{1}\right) y_{G}^{3}\left(z_{2}\right) y_{G}^{3}\left(z_{3}\right)}-\right. \\
-\frac{\alpha}{2 y\left(z_{1}\right) y\left(z_{2}\right) y\left(z_{3}\right)}\left(\frac{z_{2} z_{3}+4 S}{y\left(z_{2}\right)^{2} y\left(z_{3}\right)^{2}}+\frac{z_{1} z_{3}+4 S}{y\left(z_{1}\right)^{2} y\left(z_{3}\right)^{2}}+\frac{z_{2} z_{1}+4 S}{y\left(z_{2}\right)^{2} y\left(z_{1}\right)^{2}}\right)+ \\
\left.+\frac{1}{2 y\left(z_{1}\right) y\left(z_{2}\right) y\left(z_{3}\right)}\left(\frac{1}{y\left(z_{1}\right)^{2}}+\frac{1}{y\left(z_{3}\right)^{2}}+\frac{1}{y\left(z_{3}\right)^{2}}\right)-\frac{\check{R} \alpha}{2 y\left(z_{1}\right) y\left(z_{2}\right) y\left(z_{3}\right)}\right) v^{\prime}\left(z_{1}\right) v^{\prime}\left(z_{2}\right) v^{\prime}\left(z_{3}\right) d z_{1} d z_{2} d z_{3}= \\
=2 S\left(3 \widetilde{S}_{0}^{2} \widetilde{T}_{0}+4 S \widetilde{T}_{0}^{3}\right)-\frac{3 \alpha T-1}{2}\left(\widetilde{S}_{0}^{2}+4 S \widetilde{T}_{0}^{2}\right)+\frac{3 \alpha^{2} T_{-1}^{2} \widetilde{T}_{0}}{2}-\frac{\check{R} \alpha T_{-1}^{3}}{2}
\end{gathered}
$$

and

$$
\begin{aligned}
& e^{\left(t_{0}-T_{-1}\right) \frac{\partial}{\partial t_{0}} \oint \rho^{(0 \mid 3)}\left(z_{1}, z_{2}, z_{3}\right) v^{\prime}\left(z_{1}\right) v^{\prime}\left(z_{2}\right) v^{\prime}\left(z_{3}\right) d z_{1} d z_{2} d z_{3}=} \\
= & 2 S\left(3 \widetilde{S}_{0}^{2} \widetilde{T}_{0}+4 S \widetilde{T}_{0}^{3}\right)-\frac{3 \alpha t_{0}}{2}\left(\widetilde{S}_{0}^{2}+4 S \widetilde{T}_{0}^{2}\right)+\frac{3 \alpha^{2} t_{0}^{2} \widetilde{T}_{0}}{2}-\frac{\check{R} \alpha t_{0}^{3}}{2}
\end{aligned}
$$




$$
e^{\left(t_{0}-T_{-1}\right) \frac{\partial}{\partial t_{0}}} \oint \rho^{(1 \mid 1)}(z) v^{\prime}(z) d z=S \widetilde{T}_{1}-\frac{\alpha \widetilde{T}_{0}}{2}+\left(\check{R} F^{(1)}\right) t_{0}
$$

where the times $\widetilde{T}_{k}, \widetilde{S}_{k}$ are defined in (5.3), e.g.

$$
\widetilde{T}_{0}=\oint \frac{v(z) d z}{y(z)^{3}}=\frac{T_{0}}{8 S}
$$

and $\check{R} F^{(1)}, \check{R} \alpha$ are considered as independent constants parameterizing a solution $Z_{e G}$ to the Virasoro constraints. After commuting the operators

$$
\exp \left(-4 \frac{\partial}{\partial t_{0}} \frac{\partial}{\partial T_{0}}\right) \exp \left(-\frac{\alpha t_{0}^{2}}{4}\right)=\exp \left(-\frac{\alpha t_{0}^{2}}{4}+2 \alpha t_{0} \frac{\partial}{\partial T_{0}}-4 \alpha \frac{\partial^{2}}{\partial T_{0}^{2}}\right) \exp \left(-4 \frac{\partial}{\partial t_{0}} \frac{\partial}{\partial T_{0}}\right)
$$

one obtains

$$
\begin{gathered}
\exp \left(-\frac{\alpha t_{0}^{2}}{4}+2 \alpha t_{0} \frac{\partial}{\partial T_{0}}-4 \alpha \frac{\partial^{2}}{\partial T_{0}^{2}}\right) \exp \left(-4 \frac{\partial}{\partial t_{0}} \frac{\partial}{\partial T_{0}}\right) \times \\
\times \exp \left(\frac{1}{3 !} \oint \rho^{(0 \mid 3)}\left(z_{1}, z_{2}, z_{3}\right) v^{\prime}\left(z_{1}\right) v^{\prime}\left(z_{2}\right) v^{\prime}\left(z_{3}\right) d z_{1} d z_{2} d z_{3}+\oint \rho^{(1 \mid 1)}(z) v^{\prime}\right)= \\
=\frac{1}{3 !} \exp \left(-\frac{\alpha t_{0}^{2}}{4}+2 \alpha t_{0} \frac{\partial}{\partial T_{0}}-4 \alpha \frac{\partial^{2}}{\partial T_{0}^{2}}\right)\left(1+2 S\left(3 \widetilde{S}_{0}^{2} \widetilde{T}_{0}+4 S \widetilde{T}_{0}^{3}\right)-\frac{3 \alpha t_{0}}{2}\left(\widetilde{S}_{0}^{2}+4 S \widetilde{T}_{0}^{2}\right)+\right. \\
\left.+\frac{3 \alpha^{2} t_{0}^{2} \widetilde{T}_{0}}{2}-\frac{\check{R} \alpha t_{0}^{3}}{2}+\frac{3 \alpha T_{0}}{8 S}-\frac{3 \alpha^{2} t_{0}}{2 S}+3 !\left(S \widetilde{T}_{1}+\left(\check{R} F^{(1)}\right) t_{0}\right)+\ldots\right)= \\
=\frac{1}{3 !} \exp \left(-\frac{\alpha t_{0}^{2}}{4}-4 \alpha \frac{\partial^{2}}{\partial T_{0}^{2}}\right)\left(1+2 S\left(3 \widetilde{S}_{0}^{2} \widetilde{T}_{0}+4 S \widetilde{T}_{0}^{3}\right)+\frac{\alpha^{3} t_{0}^{3}}{8 S}-\frac{\check{R} \alpha t_{0}^{3}}{2}+\right. \\
\left.\quad+\frac{3 \alpha T_{0}}{8 S}-\frac{3 \alpha^{2} t_{0}}{4 S}+3 !\left(S \widetilde{T}_{1}+\left(\check{R} F^{(1)}\right) t_{0}\right)+\ldots\right)= \\
=\frac{1}{3 !} \exp \left(-\frac{\alpha t_{0}^{2}}{4}\right)\left(1+2 S\left(3 \widetilde{S}_{0}^{2} \widetilde{T}_{0}+4 S \widetilde{T}_{0}^{3}\right)+\frac{\alpha^{3} t_{0}^{3}}{8 S}-\frac{\check{R} \alpha t_{0}^{3}}{2}-\frac{3 \alpha^{2} t_{0}}{4 S}+3 !\left(S \widetilde{T}_{1}+\left(\check{R} F^{(1)}\right) t_{0}\right)+\ldots\right)= \\
=\frac{1}{3 !} \exp \left(-\frac{\alpha t_{0}^{2}}{4}\right)\left(1+\frac{3 \widetilde{S}_{0}^{2} T_{0}}{4}+\frac{T_{0}^{3}}{64 S}+3 ! S \widetilde{T}_{1} \ldots\right)\left(1+\frac{\alpha^{3} t_{0}^{3}}{8 S}-\frac{\check{R} \alpha t_{0}^{3}}{2}-\frac{3 \alpha^{2} t_{0}}{4 S}+3 !\left(\check{R} F^{(1)}\right) t_{0} \ldots\right)
\end{gathered}
$$

Therefore, one, indeed, obtains that the dependence on $t_{0}$ factories out from the decomposition formula (6.19), the function $Z\left(t_{0}\right)$ being $\exp \left(-\frac{\alpha t_{0}^{2}}{4}\right)\left(1+\frac{\alpha^{3} t_{0}^{3}}{8 S}-\frac{\check{R} \alpha t_{0}^{3}}{2}-\frac{3 \alpha^{2} t_{0}}{4 S}+3 !\left(\check{R} F^{(1)}\right) t_{0} \ldots\right)$.

\section{A2.3 Check of the decomposition formula in terms of ACKM moments}

Here we check the decomposition formula (17.17) of s.7. For the sake of simplicity, we check it starting only from the genus 2 free energy, since the genus 0 and genus 1 free energies are not polynomials even in terms of moments, see s.3, and therefore, their direct check is highly involved. The Gaussian free energy $F^{(2)}$ as a function of moments $t_{k}^{ \pm}$can be found in the previous Appendix, formula (A1.30). One can also calculate the same quantity using the decomposition formula. Using the coordinate system:

$$
\begin{gathered}
z-A_{-}=-x_{-}^{2} \\
z-A_{+}=x_{+}^{2}
\end{gathered}
$$


and proceeding with calculations described in s.7, one obtains

$$
\begin{gathered}
F^{(2)}=\frac{1}{256 d^{3}}\left(-1225 d^{2} G_{+}{ }^{-4} \tau_{2}^{+2}+100 d^{2} G_{-}{ }^{-2} G_{+}{ }^{-2} \tau_{2}^{-} \tau_{2}^{+}-100 d G_{-}{ }^{-2} G_{+}{ }^{-1} \tau_{2}^{-}\right. \\
+420 d^{3} G_{+}{ }^{-3} \tau_{4}^{+}+2030 d^{3} G_{+}{ }^{-4} \tau_{2}^{+} \tau_{3}^{+}+276 G_{-}{ }^{-1} G_{+}{ }^{-1}+2100 d^{3} G_{+}{ }^{-5} \tau_{2}^{+3}-560 d^{2} G_{-}{ }^{-3} \tau_{3}^{-} \\
-100 d G_{-}{ }^{-1} G_{+}{ }^{-2} \tau_{2}^{+}+2030 d^{3} G_{-}{ }^{-4} \tau_{2}^{-} \tau_{3}^{-}-175 G_{+}{ }^{-2}-175 G_{-}{ }^{-2}+525 d G_{+}{ }^{-3} \tau_{2}^{+} \\
\left.-1225 d^{2} G_{-}{ }^{-4} \tau_{2}^{-2}+525 d G_{-}{ }^{-3} \tau_{2}^{-}+420 d^{3} G_{-}{ }^{-3} \tau_{4}^{-}+2100 d^{3} G_{-}{ }^{-5} \tau_{2}^{-3}-560 d^{2} G_{+}{ }^{-3} \tau_{3}^{+}\right) g^{2}
\end{gathered}
$$

where

$$
d=A_{+}-A_{-}
$$

To compare it with the answer in terms of ACKM moments (A1.3), one should substitute $\tau^{ \pm}$with their expressions in terms of $t_{k}^{ \pm}$

$$
\begin{gathered}
t_{1}^{+}=-3 / 4 \frac{\tau_{1}^{-}}{\sqrt{d}} \\
t_{1}^{-}=3 / 4 \frac{\tau_{1}^{+}}{\sqrt{d}} \\
t_{2}^{+}=1 / 2\left(5 / 2 \tau_{2}^{-}-3 / 4 \frac{\tau_{1}^{-}}{d}\right) \frac{1}{\sqrt{d}} \\
t_{2}^{-}=1 / 2\left(5 / 2 \tau_{2}^{+}-3 / 4 \frac{\tau_{1}^{+}}{d}\right) \frac{1}{\sqrt{d}} \\
t_{3}^{+}=-1 / 2\left(7 / 2 \tau_{3}^{-}-5 / 4 \frac{\tau_{2}^{-}}{d}+\frac{9}{16} \frac{\tau_{1}^{-}}{d^{2}}\right) \frac{1}{\sqrt{d}} \\
t_{3}^{-}=1 / 2\left(7 / 2 \tau_{3}^{+}-5 / 4 \frac{\tau_{2}^{+}}{d}+\frac{9}{16} \frac{\tau_{1}^{+}}{d^{2}}\right) \frac{1}{\sqrt{d}} \\
t_{4}^{+}=1 / 2\left(9 / 2 \tau_{4}^{-}-7 / 4 \frac{\tau_{3}^{-}}{d}+\frac{15}{16} \frac{\tau_{2}^{-}}{d^{2}}-\frac{15}{32} \frac{\tau_{1}^{-}}{d^{3}}\right) \frac{1}{\sqrt{d}} \\
\left.\frac{15}{16} \frac{\tau_{2}^{+}}{d^{2}}-\frac{15}{32} \frac{\tau_{1}^{+}}{d^{3}}\right) \frac{1}{\sqrt{d}}
\end{gathered}
$$

Then, one immediately comes to formula (A1.3).

\section{A2.4 Check of the decomposition formula for $Z_{C} \longrightarrow Z_{K} \tilde{Z}_{K}$}

We check here the decomposition formula (8.27) for $Z_{C}(\mathfrak{t}) \longrightarrow Z_{K}(\tau) \tilde{Z}_{K}(\mathcal{T})$ up to $g^{2}$. Let us denote the contribution of degree $k$ in times into the genus $l$ free energy of the complex matrix model as $r^{(l \mid k)}$. Then,

$$
\begin{gathered}
r^{(0 \mid 3)}=\frac{1}{3 !} \oint_{\infty} \oint_{\infty} \oint_{\infty} \rho_{C}^{(0 \mid 3)}\left(z_{1}, z_{2}, z_{3}\right) v_{C}^{\Sigma}\left(z_{1}\right) v_{C}^{\Sigma}\left(z_{2}\right) v_{C}^{\Sigma}\left(z_{3}\right)= \\
=\frac{2 S^{2}}{3 !}\left(\oint_{\infty} \frac{v_{C}^{\Sigma}(z) d z}{y(z)^{3}}\right)^{3}=\frac{2 S^{2}}{3 !}\left(\oint_{a} \frac{v_{C}^{\Sigma}(z) d z}{y(z)^{3}}\right)^{3}=\frac{2 S^{2}}{3 !}\left(\frac{\tau_{0}}{(4 S)^{\frac{2}{3}}}\right)^{3}=\frac{\tau_{0}^{3}}{3 ! 8} \\
r^{(1 \mid 1)}=\oint_{\infty} \rho_{C}^{(1 \mid 1)}(z) v_{C}^{\Sigma}(z)=\left(\oint_{a}+\oint_{0}\right) \rho_{C}^{(1 \mid 1)}(z) v_{C}^{\Sigma}(z)=\frac{\tau_{1}}{16}-\frac{13 \cdot 2^{\frac{2}{3}} \tau_{0}}{512 S^{\frac{2}{3}}}+\frac{\mathcal{T}_{0}}{16 \sqrt{-4 S}}
\end{gathered}
$$




$$
\begin{gathered}
r^{(1 \mid 2)}=\frac{1}{2 !} \oint_{\infty} \oint_{\infty} \rho_{C}^{(1 \mid 2)}\left(z_{1}, z_{2}\right) v_{C}^{\Sigma}\left(z_{1}\right) v_{C}^{\Sigma}\left(z_{2}\right)=\frac{1}{2 !}\left(\oint_{a} \oint_{a}+2 \oint_{a} \oint_{0}+\oint_{0} \oint_{0}\right) \rho_{C}^{(1 \mid 2)}\left(z_{1}, z_{2}\right) v_{C}^{\Sigma}\left(z_{1}\right) v_{C}^{\Sigma}\left(z_{2}\right)= \\
=\frac{123}{16384} \frac{2^{\frac{1}{3}} \tau_{0}^{2}}{S^{4 / 3}}-\frac{21}{512} \frac{2^{2 / 3} \tau_{0} \tau_{1}}{S^{2 / 3}}+\frac{5}{32} \tau_{0} \tau_{2}+\frac{3}{64} \tau_{1}^{2}-\frac{\mathcal{T}_{0}^{2}}{64 \cdot 4 S}
\end{gathered}
$$

Note now that in the l.h.s. of the decomposition formula (8.27) there are no terms $r^{(0 \mid 1)}$ and $r^{(0 \mid 2)}$, since they are canceled by the corresponding contributions from the conjugation operator $e^{U_{C}}$. Therefore, one should compare sum of the three terms above, A2.25, (A2.26) and (A2.27) with the r.h.s. of the decomposition formula (8.27):

$$
\begin{aligned}
& e^{\hat{V}_{\text {ram }}} Z_{K}(\tau) \tilde{Z}_{K}(\mathcal{T})=\left(1-\frac{2^{\frac{2}{3}}}{16 \cdot 5 g S^{\frac{2}{3}}} \frac{\partial}{\partial \tau_{2}}+\frac{2^{\frac{1}{3}}}{512 \cdot 7 g S^{\frac{4}{3}}} \frac{\partial}{\partial \tau_{3}}-\frac{3 \cdot 2^{\frac{2}{3}}}{16 S^{\frac{2}{3}}} \frac{\partial^{2}}{\partial \tau_{0}^{2}}+\right. \\
& \left.+\frac{1}{2}\left(\frac{3 \cdot 2^{\frac{2}{3}}}{16 S^{\frac{2}{3}}} \frac{\partial^{2}}{\partial \tau_{0}^{2}}\right)^{2}+\frac{5 \cdot 2^{\frac{1}{3}}}{256 S^{\frac{4}{3}}} \frac{\partial^{2}}{\partial \tau_{0} \partial \tau_{1}}-\frac{2^{\frac{1}{3}}}{S^{\frac{1}{3}} \sqrt{-S}} \frac{\partial^{2}}{\partial \tau_{0} \partial \mathcal{T}_{0}} \ldots\right) \times \\
& \times\left(1+g\left(\frac{\tau_{0}^{3}}{3 ! 8}+\frac{\tau_{1}}{16}+g \frac{5 \tau_{0} \tau_{2}}{32}\right)+\frac{g^{2}}{2}\left(\frac{\tau_{0}^{3}}{3 ! 8}+\frac{\tau_{1}}{16}+g \frac{5 \tau_{0} \tau_{2}}{32}\right)^{2}+\right. \\
& \left.+g^{2}\left(\frac{1}{4} \tau_{0}{ }^{3} \tau_{1}+g \frac{5}{32} \tau_{0}{ }^{4} \tau_{2}+\frac{3}{64} \tau_{1}{ }^{2}+g \frac{15}{32} \tau_{0} \tau_{1} \tau_{2}+g \frac{35}{128} \tau_{0}{ }^{2} \tau_{3}+g^{2} \frac{75}{128} \tau_{0}{ }^{2} \tau_{2}{ }^{2}\right)+O\left(g^{3}\right)\right) \times \\
& \times\left(1+g \frac{\mathcal{T}_{0}}{16 \sqrt{-4 S}}-g^{2}\left(\frac{\mathcal{T}_{0}^{2}}{64 \cdot 4 S}+\frac{\mathcal{T}_{0}^{2}}{16 \cdot 16 \cdot 2 \cdot 4 S}\right)+O\left(g^{3}\right)\right)= \\
& =\text { const }+\left(-\frac{13}{512} \frac{2^{2 / 3} \tau_{0}}{S^{2 / 3}}+\frac{1}{16} \tau_{1}+\frac{1}{48} \tau_{0}^{3}+\frac{\mathcal{T}_{0}}{16 \sqrt{-4 S}}\right) g+\left(\frac{2137}{262144} \frac{2^{\frac{1}{3}} \tau_{0}^{2}}{S^{4 / 3}}+\frac{25}{512} \tau_{1}{ }^{2}-\right. \\
& -\frac{349}{8192} \frac{2^{2 / 3} \tau_{1} \tau_{0}}{S^{2 / 3}}+\frac{5}{32} \tau_{2} \tau_{0}-\frac{37}{24576} \frac{2^{2 / 3} \tau_{0}{ }^{4}}{S^{2 / 3}}+\frac{25}{768} \tau_{0}{ }^{3} \tau_{1}+\frac{t_{0}^{6}}{4608}+ \\
& \left.+\left(-\frac{13}{512} \frac{2^{2 / 3} \tau_{0}}{S^{2 / 3}}+\frac{1}{16} \tau_{1}+\frac{1}{48} \tau_{0}^{3}\right) \frac{\mathcal{T}_{0}}{16 \sqrt{-4 S}}-\left(\frac{\mathcal{T}_{0}^{2}}{64 \cdot 4 S}+\frac{\mathcal{T}_{0}^{2}}{16 \cdot 16 \cdot 2 \cdot 4 S}\right)\right) g^{2}+O\left(g^{3}\right)= \\
& =\text { const }+g\left(r^{(1 \mid 1)}+r^{(0 \mid 3)}\right)+g^{2}\left(\frac{\left(r^{(1 \mid 1)}\right)^{2}}{2 !}+r^{(1 \mid 2)}+O\left(t^{4}\right)\right)+O\left(g^{3}\right)
\end{aligned}
$$

\section{Acknowledgements}

We are grateful to I.Kostov who has provided us with an improved version of [19].

This work was partially supported by the Federal Program of the Russian Ministry of Industry, Science and Technology No 40.052.1.1.1112, by the grants RFBR 03-02-17373 (Alexandrov), RFBR 04-02-16538a (Mironov), RFBR 04-02-16880 (Morozov), by the Grant of Support for the Scientific Schools 8004.2006.2, NWO project 047.011.2004.026, INTAS grant 05-1000008-7865 and ANR-05BLAN-0029-01 project "Geometry and Integrability in Mathematical Physics" (A.M.'s). 


\section{References}

[1] A. Alexandrov, A. Mironov and A. Morozov, Partition functions of matrix models as the first special functions of string theory. I: Finite size Hermitean 1-matrix model, Int.J.Mod.Phys. A19 (2004) 4127; Teor. Mat. Fiz. 142 (2005) 419 (Theor.Math.Phys. 142 (2005) 349), hep-th/0310113.

[2] A. Morozov, Challenges of matrix models, hep-th/0502010

A. Mironov, Matrix Models vs. Matrix Integrals, Theor.Math.Phys. 146 (2005) 63, hep-th/0506158.

[3] M.L. Mehta, Random matrices, 2nd ed., Academic Press, New York, 1991

E. Brézin, C. Itzykson, G. Parisi and J.-B. Zuber, Planar diagrams, Commun.Math.Phys. 59 (1978) 35

D. Bessis, A new method in the combinatorics of the topological expansion, Commun.Math.Phys. 69 (1979) 147

D. Bessis, C. Itzykson and J.-B. Zuber, Quantum field theory techniques in graphical enumeration, Adv.Appl.Math. 1 (1980) 109

C. Itzykson and J.-B. Zuber, The planar approximation. II, J.Math.Phys. 21 (1980) 411.

[4] A. Gerasimov, A. Marshakov, A. Mironov, A. Morozov and A. Orlov, Matrix Models Of 2-D Gravity And Toda Theory, Nucl.Phys. B357 (1991) 565.

[5] A. Morozov, Integrability and matrix models, Phys.Usp.(UFN) 37 (1994) 1, hep-th/9303139 hep-th/9502091

A. Mironov, $2 d$ gravity and matrix models. I. $2 d$ gravity, Int.J.Mod.Phys. A9 (1994) 4355, hep-th/9312212; Matrix models of two-dimensional gravity, Phys.Part.Nucl. 33 (2002) 537.

[6] R. Dijkgraaf and C. Vafa, Matrix Models, Topological Strings, and Supersymmetric Gauge Theories, Nucl.Phys. B644 (2002) 3-20, hep-th/0206255 On Geometry and Matrix Models, Nucl.Phys. B644 (2002) 21-39, hep-th/0207106 Perturbative Derivation of Mirror Symmetry, hep-th/0208048.

[7] L. Chekhov and A. Mironov, Matrix models vs. Seiberg-Witten/Whitham theories, Phys.Lett. B552 (2003) 293, hep-th/0209085.

[8] H. Itoyama and A. Morozov, The Dijkgraaf-Vafa prepotential in the context of general SeibergWitten theory, Nucl.Phys. B657 (2003) 53, hep-th/0211245.

[9] H. Itoyama and A. Morozov, Experiments with the WDVV equations for the gluino-condensate prepotential: the cubic (two-cut) case, Phys.Lett. B555 (2003) 287, hep-th/0211259 Calculating Gluino-Condensate Prepotential, Prog.Theor.Phys. 109 (2003) 433, hep-th/0212032; GluinoCondensate (CIV-DV) Prepotential from its Whitham-Time Derivatives, Int.J.Mod.Phys. A18 (2003) 5889, hep-th/0301136.

[10] L. Chekhov, A. Marshakov, A. Mironov and D. Vasiliev, DV and WDVV, Phys.Lett. B562 (2003) 323, hep-th/0301071.

[11] L. Chekhov, A. Marshakov, A. Mironov and D. Vasiliev, Complex Geometry of Matrix Models, Proc. Steklov Inst.Math. 251 (2005) 254, hep-th/0506075.

[12] B. Eynard, All genus correlation functions for the hermitian 1-matrix model, JHEP 0411 (2004) 031, hep-th/0407261.

[13] A. Alexandrov, A. Mironov and A. Morozov, Solving Virasoro constraints in matrix models, Fortsch.Phys. 53 (2005) 512, hep-th/0412205 Unified description of correlators in non-Gaussian phases of Hermitean matrix model, Int.J.Mod.Phys. A21 (2006) 2481, hep-th/0412099. 
[14] L. Chekhov and B. Eynard, Hermitean matrix model free energy: Feynman graph technique for all genera, hep-th/0504116.

[15] A. Alexandrov, A. Mironov and A. Morozov, M-Theory of Matrix Models, hep-th/0605171.

[16] G. Bonnet, F. David and B. Eynard, Breakdown of universality in multi-cut matrix models, J.Phys. A33 (2000) 6739, cond-mat/0003324

A. Klemm, M. Mariño and S. Theisen, JHEP 0303 (2003) 051, Gravitational corrections in supersymmetric gauge theory and matrix models, hep-th/0211216.

[17] Yu. Makeenko, A. Marshakov, A. Mironov and A. Morozov, Continuum versus discrete Virasoro in one-matrix models, Nucl.Phys. B356 (1991) 574.

[18] L. Chekhov, Matrix Models and Geometry of Moduli Spaces, hep-th/9509001.

[19] I.K. Kostov, Conformal field theory techniques in random matrix models, hep-th/9907060 an improved version (unpublished).

[20] M.L. Kontsevich, Theory of intersections on moduli space of curves, Funk.Anal.Prilozh. 25 (1991) v.2, p.50 (in Russian).

[21] S. Kharchev, A. Marshakov, A. Mironov, A. Morozov and A. Zabrodin, Towards unified theory of $2 d$ gravity, Nucl.Phys. B380 (1992) 181-240, hep-th/9201013 Unification of All String Models with $c<1$, Phys.Lett. B275 (1992) 311-314, hep-th/9111037.

[22] S. Kharchev, A. Marshakov, A. Mironov and A. Morozov, Generalized Kontsevich Model Versus Toda Hierarchy and Discrete Matrix Models, Nucl. Phys. B397 (1993) 339, hep-th/9203043. Landau-Ginzburg Topological Theories in the Framework of GKM and Equivalent Hierarchies, Mod.Phys.Lett. A8 (1993) 1047-1062, hep-th/9208046 Generalized Kazakov-Migdal-Kontsevich Model: group theory aspects, Int.J.Mod.Phys. A10 (1995) 2015, hep-th/9312210.

[23] P. Di Francesco, C. Itzykson and J.-B. Zuber, Polynomial averages in the Kontsevich model, Commun.Math.Phys. 151 (1993) 193-219, hep-th/9206090

[24] A. Belavin, A. Polyakov, A. Schwarz and Yu. Tyupkin, Pseudoparticle Solutions Of The YangMills Equations, Phys.Lett. B59 (1975) 85-87.

[25] C. Callan, R. Dashen and D. Gross, Toward A Theory Of The Strong Interactions, Phys.Rev. D17 (1978) 2717.

[26] A. Mironov, A. Morozov and T. Tomaras, On the Need for Phenomenological Theory of P-Vortices or Does Spaghetti Confinement Pattern Admit Condensed-Matter Analogies? J.Exp.Theor.Phys. 101 (2005) 331-340.

[27] A. Gerasimov, A. Marshakov, A. Mironov, A. Morozov and A. Orlov, Matrix models of 2-D gravity and Toda theory, Nucl.Phys. B357 (1991) 565

F. David, Loop Equations And Nonperturbative Effects In Two-Dimensional Quantum Gravity, Mod.Phys.Lett. A5 (1990) 1019

A. Mironov and A. Morozov, On the origin of Virasoro constraints in matrix models: Lagrangian approach, Phys.Lett. B252 (1990) 47-52

J. Ambjørn and Yu. Makeenko, Properties Of Loop Equations For The Hermitean Matrix Model And For Two-Dimensional Quantum Gravity, Mod.Phys.Lett. A5 (1990) 1753

H. Itoyama and Y. Matsuo, Noncritical Virasoro algebra of $d<1$ matrix model and quantized string field, Phys.Lett. 255B (1991) 202.

[28] G. 't Hooft, A planar diagram theory for strong interactions, Nucl.Phys. B72 (1974) 461. 
[29] I.M. Krichever and S.P. Novikov, Algebras of Virasoro type, Riemann surfaces and the structure of soliton theory, Funct.Anal.Appl. 21 (1987) 126-142; Virasoro-Gelfand-Fuks Type Algebras, Riemann Surfaces, Operator's Theory Of Closed Strings, J.Geom.Phys. 5 (1988) 631-661 Virasoro-type algebras, Riemann surfaces and strings in Minkowsky space, Funct.Anal.Appl. 21 No.4 (1987) 294-307; Algebras of Virasoro type, the energy-momentum tensor, and operator expansions on Riemann surfaces, Funct.Anal.Appl. 23 (1989) 19-33.

[30] R. Dick, Global Expansions Of Holomorphic Differentials On Punctured Riemann Surfaces, Lett.Math.Phys. 18 (1989) 255

M. Schlichenmaier, Krichever-Novikov Algebras For More Than Two Points, Lett.Math.Phys. 19 (1990) 151; Krichever-Novikov Algebras For More Than Two Points: Explicit Generators, Lett.Math.Phys. 19 (1990) 327.

[31] A. Morozov, String Theory And The Structure Of Universal Module Space, Phys.Lett. B196 (1987) 325.

[32] E. Witten, Noncommutative Geometry And String Field Theory, Nucl.Phys. B268 (1985) 253; Interacting Field Theory Of Open Superstrings, ibid. B276 (1986) 291; Quantum Background Independence In String Theory, hep-th/9306122

B. Zwiebach, Quantum open string theory with manifest closed string factorization, Phys.Lett. B256 (1991) 22-29; Interpolating string field theories, Mod.Phys.Lett. A7 (1992) 1079-1090; Oriented open - closed string theory revisited, Annals Phys. 267 (1998) 193-248; Closed string field theory: An Introduction, hep-th/9305026

A. Sen and B. Zwiebach, A Proof of local background independence of classical closed string field theory, Nucl.Phys. B414 (1994) 649-714; Quantum background independence of closed string field theory, ibid. B423 (1994) 580-630; Background independent algebraic structures in closed string field theory, Commun.Math.Phys. 177 (1996) 305-326.

[33] A. Marshakov, A. Mironov and A. Morozov, WDVV-like equations in N=2 SUSY Yang-Mills Theory, Phys. Lett. B389 (1996) 43, hep-th/9607109, WDVV Equations from Algebra of Forms, Mod.Phys.Lett. A12 (1997) 773-787, hep-th/9701014; More Evidence for the WDVV Equations in N=2 SUSY Yang-Mills Theories, Int.J.Mod.Phys. A15 (2000) 1157-1206, hep-th/9701123

[34] V. Knizhnik, Analytic Fields On Riemannian Surfaces, Phys.Lett. B180 (1986) 247; Analytic Fields On Riemann Surfaces. 2, Comm.Math.Phys. 112 (1987) 567; Multiloop amplitudes in the theory of quantum strings and complex geometry, Sov.Phys.Uspekhi, 32 (1989) \#3, 945, in Russian Edition: vol.159, p.451

D. Lebedev and A. Morozov, Statistical Sums Of Strings On Hyperelliptic Surfaces, Nucl.Phys. B302 (1988) 163

A. Morozov, Two Loop Statsum Of Superstring, Nucl.Phys. B303 (1988) 342.

[35] N. Seiberg and E. Witten, Monopole Condensation, And Confinement In N=2 Supersymmetric Yang-Mills Theory, Nucl.Phys. B426 (1994) 19; Erratum-ibid. B430 (1994) 485-486.

[36] A. Gorsky, I. Krichever, A. Marshakov, A. Mironov and A. Morozov, Integrability and exact Seiberg-Witten solution, Phys.Lett. B355 (1995) 466-477, hep-th/9505035.

[37] R. Donagi and E. Witten, Supersymmetric Yang-Mills Theory And Integrable Systems, Nucl.Phys. B460 (1996) 299, hep-th/9510101.

[38] H. Itoyama and A. Morozov, Integrability and Seiberg-Witten theory; curves and periods, Nucl.Phys., B477 (1996) 855-877, hep-th/9511126 Prepotential and the Seiberg- Witten theory, Nucl.Phys., B491 (1997) 529-573, hep-th/9512161; Integrability and Seiberg-Witten theory, hep-th/9601168.

[39] A. Gorsky and A. Mironov, Integrable Many-Body Systems and Gauge Theories, hep-th/0011197. 
[40] A. Marshakov, A. Mironov and A. Morozov, On Equivalence of Topological and Quantum 2d Gravity, Phys. Lett. B274 (1992) 280, hep-th/9201011.

[41] C. Itzykson and J.B. Zuber, Combinatorics of the modular group. 2. The Kontsevich integrals, Int.J.Mod.Phys. A7 (1992) 5661, hep-th/9201001.

[42] J. Ambjörn, L. Chekhov and Yu. Makeenko, Higher Genus Correlators from the Hermitian OneMatrix Model, Phys.Lett. B282 (1992) 341-348, hep-th/9203009

J. Ambjörn, L. Chekhov, C.F. Kristjansen and Yu. Makeenko, Matrix Model Calculations beyond the Spherical Limit, Nucl.Phys. B404 (1993) 127-172; Erratum-ibid. B449 (1995) 681, hep-th/9302014.

[43] A. Givental, Semisimple Frobenius structures at higher genus, math.AG/0008067.

[44] T. Eguchi, Y. Yamada and S.K. Yang, On The Genus Expansion In The Topological String Theory, Rev.Math.Phys. 7 (1995) 279, hep-th/9405106.

[45] T. Eguchi and S.K. Yang, The Topological $C P^{1}$ model and the large $\mathrm{N}$ matrix integral, Mod.Phys.Lett. A9 (1994) 2893, hep-th/9407134

T. Eguchi, K. Hori and S.K. Yang, Topological sigma models and large N matrix integral, Int.J.Mod.Phys. A10 (1995) 4203, hep-th/9503017

K. Hori, Constraints for topological strings in $D \geq 1$, Nucl.Phys. B439 (1995) 395, hep-th/9411135.

[46] J.S. Song and Y.S. Song, Notes from the underground: A propos of Givental's conjecture, J.Math.Phys. 45 (2004) 4539, hep-th/0103254

A. Alexandrov, Givental formula in terms of Virasoro operators, J.Math.Phys. 44 (2003) 5268, hep-th/0205261.

[47] T.R. Morris, 2d Quantum Gravity, Multicritical Matter and Complex Matrices, FERMILABPUB-90-136-T

S. Dalley, C.V. Johnson and T. Morris, Multicritical complex matrix models and nonperturbative 2-D quantum gravity, Nucl.Phys. B368 (1992) 625

S. Dalley, C.V. Johnson, T. Morris and A. Watterstam, Unitary matrix models and 2-D quantum gravity, Mod.Phys.Lett. A7 (1992) 2753, hep-th/9206060

R. Lafrance and R.C. Meyers, Flows for rectangular matrix models, Mod.Phys.Lett. A9 (1994) 101, hep-th/9308113

P. Di Francesco, Rectangular Matrix Models and Combinatorics of Colored Graphs, Nucl.Phys. B648 (2003) 461, cond-mat/0207682

[48] J. Ambjorn, J. Jurkiewicz and Y.M. Makeenko, Multiloop Correlators For Two-Dimensional Quantum Gravity, Phys.Lett. B251 (1990) 517. 